TRANSACTIONS OF THE

AMERICAN MATHEMATICAL SOCIETY

Volume 361, Number 6, June 2009, Pages 2831-2869

S 0002-9947(09)04930-7

Article electronically published on January 22, 2009

\title{
EFFECTIVE REFINING OF BOREL COVERINGS
}

\author{
GABRIEL DEBS AND JEAN SAINT RAYMOND
}

\begin{abstract}
Given a countable family $\left(\boldsymbol{\Gamma}_{i}\right)_{i \in I}$ of additive or multiplicative Baire classes $\left(\boldsymbol{\Gamma}_{i}=\boldsymbol{\Sigma}_{\xi_{i}}^{0}\right.$ or $\left.\boldsymbol{\Pi}_{\xi_{i}}^{0}\right)$ we investigate the following complexity problem: Let $\left(A_{i}\right)_{i \in I}$ be a Borel covering of $\omega^{\omega}$ and assume that there exists some covering $\left(B_{i}\right)_{i \in I}$ with $B_{i} \subset A_{i}$ and $B_{i} \in \boldsymbol{\Gamma}_{i}$ for all $i$; can one find such a family $\left(B_{i}\right)_{i \in I}$ in $\Delta_{1}^{1}(\alpha)$ where $\alpha \in \omega^{\omega}$ is any reasonable code for the families $\left(A_{i}\right)_{i \in I}$ and $\left(\boldsymbol{\Gamma}_{i}\right)_{i \in I}$ ? The main result of the paper will give a full characterization of those families $\left(\boldsymbol{\Gamma}_{i}\right)_{i \in I}$ for which the answer is positive. For example we will show that this is the case if $I$ is finite or if all the Baire classes $\boldsymbol{\Gamma}_{i}$ are additive, but in the general case the answer depends on the distribution of the multiplicative Baire classes inside the family $\left(\boldsymbol{\Gamma}_{i}\right)_{i \in I}$.
\end{abstract}

\section{INTRODUCTION}

In this work we will be concerned in separation and reduction results. For simplicity we restrict our study to the Baire space $\omega^{\omega}$ but all the results admit straightforward extensions to arbitrary recursively presented Polish spaces (in the sense of [6]).

Let us recall that a set $A_{0}$ is said to be separated from a set $A_{1}$ by the set $B$ if $B \supset A_{0}$ and $B \cap A_{1}=\emptyset$. Thus the classical Suslin Separation Theorem asserts that any two $\boldsymbol{\Sigma}_{1}^{1}$ disjoint sets $A_{0}$ and $A_{1}$ can be separated (each from the other one) by a $\boldsymbol{\Delta}_{1}^{1}$ set. It is also well known that this result is "effective" in the sense that if $A_{0}$ and $A_{1}$ are $\Sigma_{1}^{1}$, then they can be separated by a $\Delta_{1}^{1}$ set. But a deeper effectivity feature of the separation theorem is given by the following result due to A. Louveau [3] (in which $\xi$ denotes a recursive ordinal):

Theorem 0.1 (Louveau). Let $A_{0}$ and $A_{1}$ be two disjoint $\Sigma_{1}^{1}$ sets. If $A_{0}$ is separated from $A_{1}$ by a $\boldsymbol{\Sigma}_{\xi}^{0}$ set, then $A_{0}$ is separated from $A_{1}$ by a set in $\Delta_{1}^{1} \cap \boldsymbol{\Sigma}_{\xi}^{0}$.

In fact Louveau's result asserts that $A_{0}$ can be separated from $A_{1}$ by a set in $\Sigma_{\xi}^{0}(\alpha)$ for some $\alpha \in \Delta_{1}^{1}$. Notice that this result is symmetrical: in swapping the indices 0 and 1 one obtains a similar statement for the multiplicative Baire classes $\Pi_{\xi}^{0}$.

Let us also recall that the Suslin Theorem can be extended to infinite families. This extension, which is due to Novikov, also admits the following effective version: For any $\Sigma_{1}^{1}$ family $\left(A_{i}\right)_{i \in I}$ of sets such that $\bigcap_{i \in I} A_{i}=\emptyset$ there exists a $\Delta_{1}^{1}$ family $\left(B_{i}\right)_{i \in I}$ such that $B_{i} \supset A_{i}$ for all $i$ and $\bigcap_{i \in I} B_{i}=\emptyset$.

Received by the editors January 30, 2006.

2000 Mathematics Subject Classification. Primary 03E15; Secondary 03E45, 54 H05.

Key words and phrases. Covering, separation, effectivity, Novikov Theorem, distinguished tree relations.

(C)2009 American Mathematical Society Reverts to public domain 28 years from publication 
Our goal in this work is to investigate an analog of Theorem 0.1 in the context of the Novikov Theorem. More precisely we shall consider the following:

Question. Fix a recursive (in any obvious sense) family $\left(\boldsymbol{\Gamma}_{i}\right)_{i \in I}$ of additive or multiplicative Baire classes. Let $\left(A_{i}\right)_{i \in I}$ be a $\Sigma_{1}^{1}$ family of sets such that $\bigcap_{i \in I} A_{i}=$ $\emptyset$, and suppose that there exists a family $\left(B_{i}\right)_{i \in I}$ of Borel sets such that $B_{i} \in \boldsymbol{\Gamma}_{i}$, $B_{i} \supset A_{i}$ for all $i$, and $\bigcap_{i \in I} B_{i}=\emptyset$. Can one find such a family $\left(B_{i}\right)_{i \in I}$ which is moreover $\Delta_{1}^{1}$ ?

We shall prove that the answer to this question is positive if we restrict ourselves to finite families but might be negative in the general case. In fact the main result of the paper will give a full characterization of those families $\left(\boldsymbol{\Gamma}_{i}\right)_{i \in I}$ for which the answer is positive. Surprisingly in this characterization the precise nature (additive or multiplicative) of the Baire classes $\boldsymbol{\Gamma}_{i}$ will be crucial. In particular, and unlike in the case of Theorem 0.1 this result will not be symmetrical, and one cannot replace freely some Baire class $\boldsymbol{\Gamma}_{i}$ by its dual class.

In fact we shall derive the main result from a general dichotomy for infinite families of $\Sigma_{1}^{1}$ sets, which extends the following strengthening of Theorem 0.1 proved in [4]:

Theorem 0.2 (Louveau - Saint Raymond). Fix a $\Pi_{\xi}^{0}$ subset $B_{0}$ of $2^{\omega}$. Then for any $\Sigma_{1}^{1}$ disjoint sets $A_{0}$ and $A_{1}$ in $\omega^{\omega}$ at least one of the following alternatives holds:

- either $A_{0}$ is separated from $A_{1}$ by a set in $\Sigma_{\xi}^{0}(\alpha)$ for some $\Delta_{1}^{1}$ real $\alpha$, - or there exists a continuous mapping $f: 2^{\omega} \rightarrow \omega^{\omega}$ such that $B_{0}=f^{-1}\left(A_{0}\right)$ and $2^{\omega} \backslash B_{0}=f^{-1}\left(A_{1}\right)$.

(To recover Theorem 0.1 apply Theorem 0.2 to any $B_{0} \in \Pi_{\xi}^{0} \backslash \Sigma_{\xi}^{0}$ and observe that if $A_{0}$ is separated from $A_{1}$ in $\boldsymbol{\Sigma}_{\xi}^{0}$, then the second alternative cannot hold.) But as we shall see, a nonnegligible part of the difficulty in the new dichotomy result will already lie in its formulation which will be less natural than the statement of Theorem 0.2 In particular in the second alternative one needs to consider some less intuitive notion of reduction.

From now on we shall deal with the dual formulation of all these results, replacing sets by their complements. In this duality, families with empty intersection are thus replaced by coverings. This choice is of course completely arbitrary and nonfundamental.

\section{BASIC NOTIONS AND NOTATION}

1.1. General notation. Since many arguments of the paper refer to our previous work [1] we shall try to keep following the same basic notation as long as this is possible. In particular we denote by $\operatorname{Seq}(E)$ the set of all finite sequences in a given set $E$. This notation will be more convenient than the more classical $E^{<\omega}$ since we will be very often working in spaces such as $\operatorname{Seq}(\operatorname{Seq}(\omega))$ (and much worth in 1).

The inclusion relation $\subset$ should always be understood in the nonstrict sense (so $a \subset a)$. However when working in $\operatorname{Seq}(E)$ we will use more specific notation: " $\prec$ " will denote the strict standard extension relation, while the nonstrict extension relation will be denoted by " $\preceq$ ". For this latter relation we shall also use the notation "Ext" introduced in 1 .

The complement of a subset $A$ of the set $X$ will be denoted by $A^{c}$ or by $X \backslash A$.

The disjoint union of two sets $A$ and $B$ will be denoted by $A+B$. 
1.2. Descriptive classes. We shall mainly consider the classical descriptive classes: $\boldsymbol{\Delta}_{1}^{1}, \boldsymbol{\Sigma}_{1}^{1}, \boldsymbol{\Pi}_{1}^{1}, \ldots$ and their "effective" versions: $\Delta_{1}^{1}, \Sigma_{1}^{1}, \Pi_{1}^{1}, \ldots$, as well as their "relativization" to some real: $\Delta_{1}^{1}(\alpha), \Sigma_{1}^{1}(\alpha), \Pi_{1}^{1}(\alpha), \ldots$ (See [6] for more details.)

However we emphasize that by a Baire class of $\operatorname{rank} \xi$, where $1 \leq \xi<\omega_{1}$, we will always mean the classical additive (boldface) Baire class $\boldsymbol{\Sigma}_{\xi}^{0}$ or the multiplicative (boldface) Baire class $\boldsymbol{\Pi}_{\xi}^{0}$. Also to avoid any possible confusion we shall never consider the "effective" (lightface) classes $\Sigma_{\xi}^{0}$ or $\Pi_{\xi}^{0}$ in the sequel.

Borel mappings will be analyzed by the inverse image operation. So we shall say that the Borel mapping $f: X \rightarrow Y$ is of class $\xi$ if the inverse image of any open set is in $\boldsymbol{\Sigma}_{1+\xi}^{0}$. We recall that this notion is finer than the notion of Baire class $\xi$ obtained by taking (as in [2] or [6]) iterated limits of continuous functions. In fact by a clasical result of Lebesgue and Hausdorff there is a one-to-one correspondence between the notion of "Baire class" and the notion of "Borel successor class" (see [2], Theorem 24.3). But if $\lambda$ is limit the Baire iterated limit procedure does not distinguish between mappings of class $\lambda$ and those of class $\lambda+1$.

1.3. Codes. We shall make frequent use of various notions of codes. By definition a code will be an element of $\omega^{\omega}$. All these notions are natural variations of classical codings and we shall not make them explicit. For example a code for an ordinal $\xi$ will just be an element of the classical space WO (see [6]). Similarly a code for a Baire class $\boldsymbol{\Gamma}$ of rank $\xi$ will be a real $\alpha \approx(i, \beta) \in 2 \times \mathbf{W O}$ where $i=0,1$ indicates whether $\boldsymbol{\Gamma}$ is additive or multiplicative and $\beta$ is a code for $\xi$. Also given any notion of code for the elements of some set $A$ and $\xi$ any ordinal, then a code for the family $\left(a_{\eta}\right)_{\eta<\xi} \in A^{\xi}$ will be real $\gamma \approx\left(\left(\alpha_{n}\right)_{n \in \omega}, \beta\right)$ with $\beta \in \mathbf{W O}$ a code for $\xi$ and such that for all $n, \alpha_{n}$ is a code for $a_{\eta}$ where $\eta$ is the order type of $\beta_{\left.\right|_{n}}$.

\section{MAIN RESUltS}

We first introduce two basic notions needed for stating our main results.

2.1. Baire types. A Baire type is simply a family $\left(\boldsymbol{\Gamma}_{i}\right)_{i \in I}$ where each $\boldsymbol{\Gamma}_{i}$ is a Baire class. If for all $i \in I, \boldsymbol{\Gamma}_{i}$ is of rank $\xi_{i}$ we shall say that the Baire type $\left(\boldsymbol{\Gamma}_{i}\right)_{i \in I}$ is of $\operatorname{rank}\left(\xi_{i}\right)_{i \in I}$.

We shall say that a family $\left(A_{i}\right)_{i \in I}$ of Borel sets is of Baire type (or simply of type) $\left(\boldsymbol{\Gamma}_{i}\right)_{i \in I}$ if for all $i \in I, A_{i} \in \boldsymbol{\Gamma}_{i}$.

For practical reasons it will be convenient to work with families $\left(A_{i}\right)_{i \in I}$ of sets with an "abstract" index set $I$; however when we use any descriptive notion in this context it will be understood that $I$ is a subset of some (discrete) recursive space such as $\omega$ or $\omega^{n}$.

Definition 2.2. Given two families $\left(A_{i}\right)_{i \in I}$ and $\left(B_{i}\right)_{i \in I}$ of subsets of some space $X$, we shall say that $\left(B_{i}\right)_{i \in I}$ is subordinate to $\left(A_{i}\right)_{i \in I}$ if:

$$
\bigcup_{i \in I} B_{i}=\bigcup_{i \in I} A_{i} \quad \text { and } \quad \forall i \in I, \quad B_{i} \subset A_{i} .
$$

We shall very often use this notion when $\left(A_{i}\right)_{i \in I}$ is a covering of $X$, in which case a subordinate family $\left(B_{i}\right)_{i \in I}$ is automatically a covering too and we shall then say that $\left(B_{i}\right)_{i \in I}$ is a subordinate covering to $\left(A_{i}\right)_{i \in I}$. This notion is of quite common use (under different terminologies) in analysis, namely in connection with the so-called "partitions of unity". But our main concern here is that the Novikov 
Theorem or more precisely its dual formulation, that is, the "Reduction Theorem", can be expressed by simply saying that any $\boldsymbol{\Pi}_{1}^{1}$ covering $\left(A_{i}\right)_{i \in I}$ admits a Borel subordinate covering $\left(B_{i}\right)_{i \in I}$. Observe however that we do not impose on the Borel sets $B_{i}$ in the subordinate covering to be pairwise disjoint; this detail is very important.

Let us also emphasize here that a subordinate family has the same index set as the initial family. In particular the notion of subordination is totally different from the notion of "refinement" considered in general topology in which one compares coverings $\left(A_{i}\right)_{i \in I}$ and $\left(B_{j}\right)_{j \in J}$ with possibly different index sets.

We shall now state the main result of this work. This result concerns in fact very special Baire types that we will call "adequate" (see Definition 6.2). This totally ad hoc notion is not very intuitive, and it would be very difficult to motivate it at this level of the discussion. Let us only point out that a Baire type with only finitely many multiplicative Baire classes is adequate. Conversely in an adequate Baire type a multiplicative Baire class can occur at most finitely many times, though infinitely many different multiplicative Baire classes might occur but then in some very specific configuration.

From now on whenever the surrounding space is not specified it will be implicitly assumed to be $\omega^{\omega}$.

Theorem A. Let $\left(\boldsymbol{\Gamma}_{i}\right)_{i \in I}$ be a Baire type with code $\alpha \in \omega^{\omega}$. Then the following are equivalent:

(i) For all $\beta \in \omega^{\omega}$, any $\Pi_{1}^{1}(\beta)$ covering which admits a subordinate covering of type $\left(\boldsymbol{\Gamma}_{i}\right)_{i \in I}$ admits a $\Delta_{1}^{1}(\alpha, \beta)$ subordinate covering of type $\left(\boldsymbol{\Gamma}_{i}\right)_{i \in I}$.

(ii) For all $\beta \in \omega^{\omega}$, any $\Delta_{1}^{1}(\beta)$ covering which admits a subordinate covering of type $\left(\boldsymbol{\Gamma}_{i}\right)_{i \in I}$ admits a $\Delta_{1}^{1}(\alpha, \beta)$ subordinate covering of type $\left(\boldsymbol{\Gamma}_{i}\right)_{i \in I}$.

(iii) $\left(\boldsymbol{\Gamma}_{i}\right)_{i \in I}$ is an adequate Baire type.

So $(i)$ asserts that the answer to our initial question is positive, and (iii) gives the necessary and sufficient condition on $\left(\boldsymbol{\Gamma}_{i}\right)_{i \in I}$ for this.

To prove Theorem A it is clearly sufficient to prove that $(i i) \Rightarrow(i i i) \Rightarrow(i)$. Both implications are nontrivial. But the first one is much simpler and will be derived from a general complexity argument: assuming that $\left(\boldsymbol{\Gamma}_{i}\right)_{i \in \omega}$ is not adequate we shall prove that (via some suitable coding) the set of all $\Delta_{1}^{1}(\beta)$ coverings which admit a subordinate covering of type $\left(\boldsymbol{\Gamma}_{i}\right)_{i \in \omega}$ is $\boldsymbol{\Sigma}_{1}^{1}$-hard (i.e. reduces continuously any $\boldsymbol{\Sigma}_{1}^{1}$ set). It will follow that this set cannot coincide with the set of all $\Delta_{1}^{1}(\beta)$ coverings admitting a $\Delta_{1}^{1}(\alpha, \beta)$-subordinate covering of type $\left(\boldsymbol{\Gamma}_{i}\right)_{i \in \omega}$, since the latter is $\Pi_{1}^{1}(\alpha, \beta)$. In particular the argument will not construct any explicit $\beta$ and the universal quantifications in the statements of $(i)$ and $(i i)$ are crucial to get the equivalence with $(i i i)$.

The proof of the second implication $(i i i) \Rightarrow(i)$ is much more elaborate and will follow from a general dichotomy concerning $\Pi_{1}^{1}$ coverings, which can be viewed as a generalization of Theorem 0.2 To state simply this result we need to introduce one more notion.

Definition 2.3. Let $\left(A_{i}\right)_{i \in I}$ be a family of subsets of some space $X$ and $\left(B_{i}\right)_{i \in I}$ be a family of subsets of some space $Y$. We shall say that $\left(A_{i}\right)_{i \in I}$ is weakly reducible to the family $\left(B_{i}\right)_{i \in I}$ of subsets of $Y$ if there exists a continuous mapping $f: X \rightarrow Y$ 
such that:

$$
\forall x \in \bigcup_{i \in I} A_{i}, \exists i_{*} \in I, x \in A_{i_{*}} \text { and } f(x) \in B_{i_{*}} .
$$

We shall then say that $f$ is a weak reduction of $\left(A_{i}\right)_{i \in I}$ to $\left(B_{i}\right)_{i \in I}$.

Observe that if $\left(A_{i}\right)_{i \in I}$ is a partition, then a weak reduction reduces (in the classical sense) each $A_{i}$ to $B_{i}$. However for our main application we shall need to deal with families which cannot be pairwise disjoint.

The proof of the following result constitutes the major content of the paper.

Theorem B. For any $\alpha \in \omega^{\omega}$, if $\left(\boldsymbol{\Gamma}_{i}\right)_{i \in I}$ is an adequate Baire type with code in $\Delta_{1}^{1}(\alpha)$ and $\left(B_{i}\right)_{i \in I}$ is a $\Delta_{1}^{1}(\alpha)$ family of type $\left(\boldsymbol{\Gamma}_{i}\right)_{i \in I}$, then for any $\Pi_{1}^{1}(\alpha)$ covering $\left(C_{i}\right)_{i \in I}$ at least one of the following two alternatives holds:

- either $\left(C_{i}\right)_{i \in I}$ admits a $\Delta_{1}^{1}(\alpha)$-subordinate covering of type $\left(\boldsymbol{\Gamma}_{i}\right)_{i \in I}$,

- or $\left(B_{i}\right)_{i \in I}$ is weakly reducible to $\left(\omega^{\omega} \backslash C_{i}\right)_{i \in I}$.

In fact the real starting point of the present work goes back to the alternative proof of Theorem 0.2 that we gave recently in [1. At that time this proof was conceived merely as an additional illustration for the use of the representation of Borel sets developed in that work. In both proofs, in [4 as well as in 1], the dichotomy corresponds to the determinacy of some suitable open game which is introduced. However while the whole difficulty in the initial proof in [4] lies precisely in the definition of the game, the game considered in [1, which relies on the Representation Theorem of Borel sets, is very simple and quite natural.

Also to prove Theorem B we shall follow the same scheme. But unfortunately in the present case the obstacles will be numerous. In particular we will need to ameliorate the representation of Borel sets developed in 11, which is suitable to analyze a countable family of Borel sets of the same Borel rank, but is not adapted to handle Borel sets of variable rank. Also in the new context the definition of the game as well as the arguments present serious new technical difficulties, and it is precisely to overcome these difficulties that we were led to consider "adequate" Baire types, which happened to be the optimal setting. In particular the arguments for additive and multiplicative Baire classes will not be symmetrical as in the proof of Theorem 0.2. Less expected are the additional problems which appear in the treatment of multiplicative Baire classes of limit rank.

Theorem B, from which follows the implication $($ iii $) \Rightarrow(i)$ of Theorem A, will be proved in Section 7, which is the core of this work. This proof will make use of a large amount of material from [1] (more specifically Chapters I and III). In fact the situation is a bit more complicated since the results of [1] are not adapted to our needs and we will have to prove new versions which are not merely generalizations, though the basic ideas and concepts are extracted from [1. In particular we will need a new version of the Representation Theorem for Borel sets proved in [1]. This result is presented in Section 3, however its proof, which is rather technical and does not really interact with the rest of this work, is postponed to Section 9 , In Section 4 we shall present another basic result from [1] that we shall then extend in Section [5. In Section [6 we introduce the last ingredient, which is the notion of adequate Baire type, and prove some elementary properties.

The second implication $(i i) \Rightarrow($ iii $)$ of Theorem A, which proves the necessity of the "adequate Baire type" assumption in Theorem B, is established in Section 8. This section is totally independent from the rest of the paper. 
Despite the high interference of several results from [1] in the present work the paper is essentially self-contained. In particular all basic notions are made explicit and widely commented upon and unless the reader wants to check some proofs he should normally not need to go back to our earlier work.

\section{The Representation Theorem for Borel Sets}

In this section we state without proof the Representation Theorem for Borel sets, which is the main ingredient of the proof of the Dichotomy Theorem. For this we need to introduce first the basic notion of distinguished subrelation which is at the heart of this representation. However the reader who is only interested in the proof of the Dichotomy Theorem can find at the end of the present section (Remark 3.13) a statement which avoids the nonintuitive notion of distinction.

3.1. Tree relations. Let Ext denote the (nonstrict) extension relation on Seq $(\omega)$ (see 1.1). The Representation Theorem (for Borel subsets of $\omega^{\omega}$ ) involves relations $R$ on $\operatorname{Seq}(\omega)$ which are finer than Ext, so:

$$
R \subset \operatorname{Ext} \subset \operatorname{Seq}(\omega) \times \operatorname{Seq}(\omega) .
$$

However it will be clearer for the exposition to consider these notions in a general setting. Let us recall that a relation $R$ on a set $E$ is a tree relation if :

(1) $R$ is a partial order on $E$.

(2) $R$ admits a least element.

(3) Any initial segment of $R$ is finite and linearly ordered.

By the predecessor of an element $s$ in $E=\operatorname{Dom}(R)$ we will always mean the strict predecessor of $s$. The number of predecessors of $s$ is called the height of $s$, and will be denoted by $h_{R}(s)$. So the height of the least element is 0 .

We will denote by $[R]$ the set of all infinite $R$-branches, that is, the maximal (relative to the inclusion relation) linearly ordered subsets of $\operatorname{Dom}(R)$. We also equip $[R]$ with the topology generated by the sets:

$$
N_{s}^{R}=\{\sigma \in[R]: \quad s \in \sigma\}
$$

for $s \in \operatorname{Dom}(R)$. This topology is Polish if $E$ is countable and the sets $N_{s}^{R}$ are actually clopen and form a basis for this topology $\left(N_{s}^{R} \cap N_{t}^{R}=\emptyset\right.$ or $N_{s}^{R} \subset N_{t}^{R}$ or $\left.N_{t}^{R} \subset N_{s}^{R}\right)$. Observe that Ext-branches are just the sets of the form

$$
\hat{x}:=\left\{x_{\left.\right|_{n}}: n \in \omega\right\} \subset \operatorname{Seq}(\omega)
$$

for $x \in \omega^{\omega}$, and the mapping $x \mapsto \hat{x}$ is obviously a homeomorphism from $\omega^{\omega}$ onto [Ext].

3.2. Canonical mappings. If $R_{1} \subset R_{0}$ are two tree relations, then any infinite $R_{1}$-branch $\sigma$ is contained in a unique infinite $R_{0}$-branch $\pi(\sigma)$; this defines a canonical mapping:

$$
\pi:\left[R_{1}\right] \rightarrow\left[R_{0}\right]
$$

This mapping is clearly continuous but a priori it is neither injective nor surjective. The essence of the Representation Theorem is to associate to any $\boldsymbol{\Sigma}_{1+\xi}^{0}$ set $B \subset$ $\omega^{\omega} \approx$ [Ext], a tree relation $R \subset$ Ext such that:

(1) the canonical mapping $\pi:[R] \rightarrow[$ Ext $]$ is bijective,

(2) the inverse mapping $\pi^{-1}:[\mathrm{Ext}] \rightarrow[R]$ is Borel of class $\xi$,

(3) $\pi^{-1}(B)$ is $\boldsymbol{\Sigma}_{1}^{0}$. 
But let us point out that behind the descriptive statement (2) lies a purely algebraic property, that we introduce now.

Definition 3.3. Let $R_{1} \subset R_{0}$ be two tree relations. $R_{1}$ is said to be distinguished in $R_{0}$ if for all $u R_{0} v R_{0} w$ the following implication holds:

$$
u R_{1} w \Longrightarrow u R_{1} v \text {. }
$$

In particular if $R_{1}$ is distinguished in $R_{0}$, then for any $v$ such that $v R_{0} w$, the $R_{1}$-predecesssor $w^{*}$ of $w$ is already an $R_{1}$-predecesssor of $v$, unless $v R_{0} w^{*}$. In fact this last property is equivalent to the distinction of $R_{1}$ in $R_{0}$ and is the "concrete" way in which this notion will be used in most applications.

As a trivial example observe if a relation $R$ depends only on the first argument, that is, if for all $u \neq v$ and $u \neq w$ we have

$$
u R v \Longleftrightarrow u R w
$$

then $R$ is distinguished in any coarser relation $R_{0} \supset R$. But in this case we can do much better: for any other relation $R_{1} \subset R_{0}$, if $R_{1}$ distinguished in $R_{0}$, then $R_{1} \cup R$ is distinguished in $R_{0}$. This simple observation will play a fundamental role in Section 5. But of course the proof of the Representation Theorem in Section 9 will provide other nontrivial examples.

The following consequence of Theorem I.3.8 in [1] will give a first indication on the descriptive aspect hidden behind the algebraic notion of distinction.

Proposition 3.4. If $R_{1}$ is distinguished in $R_{0}$, then the canonical mapping $\pi$ : $\left[R_{1}\right] \rightarrow\left[R_{0}\right]$ is one-to-one. If moreover $\pi$ is onto, then the inverse mapping $\pi^{-1}$ : $\left[R_{0}\right] \rightarrow\left[R_{1}\right]$ is Borel of class one.

Consider now a finite chain of tree relations:

$$
R^{(n)} \subset R^{(n-1)} \subset \cdots \subset R^{(0)}
$$

and suppose that each relation $R^{(k)}$ is distinguished in the previous one. The canonical mapping $\pi_{n}:\left[R^{(n)}\right] \rightarrow\left[R^{(0)}\right]$ is then just the composition of the successive canonical mappings $\pi_{(k, k-1)}:\left[R^{k}\right] \rightarrow\left[R^{(k-1)}\right]$. Hence it follows from Proposition 3.4 that $\pi_{n}$ is also one-to-one. Moreover if $\pi_{n}$ is onto, then all the $\pi_{k}$ are also onto; hence, again by Proposition $\left[3.4\right.$, the mapping $\pi_{n}^{-1}:\left[R^{(0)}\right] \rightarrow\left[R^{(n)}\right]$ is Borel of class $n$.

Unfortunately these simple arguments do not extend to an infinite chain and in this case the situation is much more delicate.

Definition 3.5. A transfinite chain of tree relations $\left(R^{(\eta)}\right)_{\eta \leq \xi}$ is said to be a resolution family if:

1) For all $\eta<\xi, R^{(\eta+1)} \subset R^{(\eta)}$ and $R^{(\eta+1)}$ is distinguished in $R^{(\eta)}$.

2) For all $\lambda \leq \xi$ limit, $R^{(\lambda)}=\bigcap_{\eta<\lambda} R^{(\eta)}$.

Observe that if $\left(R^{(\eta)}\right)_{\eta \leq \xi}$ is a resolution family, then it follows from Proposition 3.4 that all the canonical mappings $\pi_{\eta}:\left[R^{(\eta)}\right] \rightarrow\left[R^{(0)}\right]$ are one-to-one.

Definition 3.6. A resolution family $\left(R^{(\eta)}\right)_{\eta \leq \xi}$ is said to be an expansion family if the last canonical mapping $\pi_{\xi}$ is onto; in this case all the canonical mappings $\pi_{\eta}$ are bijective. 
Thus if $\left(R^{(\eta)}\right)_{\eta \leq \xi}$ is an expansion family and $\eta \leq \xi$, then any $R^{(0)}$-infinite branch $\sigma$ contains a unique $R^{(\eta)}$-infinite branch $\pi_{\eta}^{-1}(\sigma)$ that we shall also denote by $\sigma^{(\eta)}$. The following simple result, which is not stated explicitly in [1, will be enlightening for the sequel.

Proposition 3.7. If $\left(R^{(\eta)}\right)_{\eta \leq \xi}$ is an expansion family, then

$$
\begin{cases}\sigma^{(\eta+1)}=\left\{s \in \sigma^{(\eta)}: \forall t \in \sigma^{(\eta)},\left(s R^{(\eta)} t \Longrightarrow s R^{(\eta+1)} t\right)\right\} & \text { if } \eta<\xi, \\ \sigma^{(\lambda)}=\bigcap_{\eta<\lambda} \sigma^{(\eta)} & \text { if } \lambda \leq \xi \text { is limit. }\end{cases}
$$

Proof. Since $R^{(\eta+1)}$ is distinguished in $R^{(\eta)}$ the successor case follows from Corollary I-3.6 in [1], and the limit case is obvious.

Let $\mathcal{R}=\left(R^{(\eta)}\right)_{\eta \leq \xi}$ be a given expansion family. To compute the complexity of the mapping $\pi_{\eta}^{-1}:\left[R^{(0)}\right] \rightarrow\left[R^{(\eta)}\right]$ consider a basic clopen set $N_{s}^{R^{(\eta)}}$ of the space $\left[R^{(\eta)}\right]$ and observe that

$$
\pi_{\eta}\left(N_{s}^{R^{(\eta)}}\right)=\left\{\sigma \in\left[R^{(0)}\right]: s \in \sigma^{(\eta)}\right\} .
$$

Then using Proposition 3.7 one can show by a straightforward induction that the set $\pi_{\eta}\left(N_{s}^{R^{(\eta)}}\right)$ is $\boldsymbol{\Delta}_{\eta+1}^{0}$; hence the image by $\pi_{\eta}$ of any $\boldsymbol{\Sigma}_{1}^{0}$ set is a $\boldsymbol{\Sigma}_{\eta+1}^{0}$ set. In particular if $\eta$ is finite, then $\pi_{\eta}^{-1}$ is of class $\eta$ as was already observed above, but if $\eta$ is infinite, this only proves that $\pi_{\eta}^{-1}$ is of class $\eta+1$ (for the notion of class of a Borel mapping see 1.2). However it is proved in [1] (Proposition I-4.1) that when $\xi$ is limit, then, under some additional "uniformity" assumption on $\mathcal{R}$, the last inverse mapping $\pi_{\xi}^{-1}$ is of Baire class $\xi$; but even in this case the mappings $\pi_{\eta}^{-1}$ for $\eta<\xi$ are still of class $\eta+1$.

Moreover the previous complexity bounds are best possible since by the Representation Theorem proved in [1] (Theorem I-6.6) any $\boldsymbol{\Sigma}_{\xi}^{0}$ set $B$ can be obtained as the image of some $\boldsymbol{\Sigma}_{1}^{0}$ set by the last canonical mapping associated to some expansion family $\mathcal{R}=\left(R^{(\eta)}\right)_{\eta \leq \xi^{\prime}}$ with either $\xi=\xi^{\prime}+1$, or $\xi=\xi^{\prime}$ limit and $\mathcal{R}$ "uniform".

Nevertheless we shall prove in the present work that if one strengthens the uniformity condition, then all the inverse mappings $\pi_{\eta}^{-1}$ become of Baire class $\eta$ (instead of $\eta+1$ ), and it will follow from the new Representation Theorem (Theorem 3.10 below) that any $\boldsymbol{\Sigma}_{\xi}^{0}$ set $B$ can be obtained as the image of some $\boldsymbol{\Sigma}_{1}^{0}$ set by the last canonical mapping associated to some expansion family $\mathcal{R}=\left(R^{(\eta)}\right)_{\eta \leq \xi}$ satisfying this stronger uniformity condition. However this new version of the Representation Theorem is formally incomparable with the one proved in [1] since the latter provides (in the successor case) representations with shorter resolution families, and this specific detail is crucial for the proof of the main result of [1] (Theorem VI-0.1).

Before we introduce the next basic notion observe that if $\left(R^{(\eta)}\right)_{\eta \leq \xi}$ is a resolution family and $\lambda \leq \xi$ is limit, then it follows from the continuity condition at $\lambda$ that:

$$
\forall v, \exists \eta<\lambda, \forall u\left(u R^{(\eta)} v \Rightarrow u R^{(\lambda)} v\right) .
$$

Definition 3.8. a) We shall say that the resolution family $\left(R^{(\eta)}\right)_{\eta \leq \xi}$ is strongly continuous at the limit ordinal $\lambda \leq \xi$ if:

$$
\forall u, \exists \eta<\lambda, \forall v\left(u R^{(\eta)} v \Rightarrow u R^{(\lambda)} v\right) .
$$

b) We shall say that $\left(R^{(\eta)}\right)_{\eta \leq \xi}$ is strongly continuous if it is strongly continuous at any limit ordinal $\lambda \leq \xi$. 
The following result that we shall not use can be derived directly from Proposition 3.7, but it can also (see Remark 4.5) be interpreted as a particular case of Theorem 4.10 proved in the next section.

Proposition 3.9. If $\left(R^{(\eta)}\right)_{\eta \leq \xi}$ is a strongly continuous expansion family, then for all $\eta \leq \xi$ the mapping $\pi_{\eta}^{-1}:\left[R^{(0)}\right] \rightarrow\left[R^{(\eta)}\right]$ is Borel of class $\eta$.

We can now state the Representation Theorem that we will prove in Section 9.

Theorem 3.10. Let $\left(B_{i}\right)_{i \in I}$ be a countable family of Borel subsets of $\omega^{\omega}$ with $B_{i} \in$ $\Sigma_{1+\xi_{i}}^{0}$, and set $\xi=\sup _{i \in I} \xi_{i}$. Then there exists a strongly continuous expansion family $\left(R^{(\eta)}\right)_{\eta \leq \xi}$ with $R^{(0)}=$ Ext such that for all $i \in I, \pi_{\xi_{i}}^{-1}\left(B_{i}\right)$ is a $\Sigma_{1}^{0}$ subset of $\left[R^{\left(\xi_{i}\right)}\right]$.

In the proof of the Dichotomy Theorem in Section 7 we will need to apply this result to a family $\left(B_{i}\right)_{i \in \omega}$ of Borel sets of Baire type $\left(\boldsymbol{\Gamma}_{i}\right)_{i \in I}$. Of course taking complements if necessary one can always apply Theorem 3.10 to get a resolution family $\left(R^{(\eta)}\right)_{\eta \leq \xi}$ such that $B_{i}^{\prime}:=\pi_{\xi_{i}}^{-1}\left(B_{i}\right)$ is either open or closed, depending on whether $\boldsymbol{\Gamma}_{i}$ is additive or multiplicative. But for technical reasons we will prefer to work with a slightly more concrete such representation.

Definition 3.11. Let $R$ be a tree relation and $S \subset \operatorname{Dom}(R)$.

a) We shall say that $S$ is $R$-hereditary if

$$
(u R v \text { and } v \in S) \Longrightarrow u \in S \text {. }
$$

b) We shall say that $S$ is $R$-monotone if $\operatorname{Dom}(R) \backslash S$ is $R$-hereditary, that is, if

$$
(u R v \text { and } u \in S) \Longrightarrow v \in S \text {. }
$$

It is quite clear that $R$-hereditary sets are the analog of trees (which are precisely the Ext-hereditary sets). For example one can easily see that for any $\Pi_{1}^{0}$ subset $A$ of $[R]$ there exists an $R$-hereditary set $S \subset \operatorname{Dom}(R)$ such that for all $\sigma \in[R]$ :

$$
\sigma \in A \Longleftrightarrow \sigma \subset S
$$

and by duality, for any $\boldsymbol{\Sigma}_{1}^{0}$ subset of $[R]$ there exists an $R$-monotone set $S \subset \operatorname{Dom}(R)$ such that for all $\sigma \in[R]$ :

$$
\sigma \in A \Longleftrightarrow \sigma \cap S \neq \emptyset \text {. }
$$

Moreover if $A$ is $\Delta_{1}^{1}(\alpha)$ for some $\alpha$, then one can find $S$ in $\Delta_{1}^{1}(\alpha)$ : this can be easily derived from the classical case $R=$ Ext.

We can now restate Theorem 3.10 in a more adapted way for our application.

Theorem 3.12. Let $\left(\boldsymbol{\Gamma}_{i}\right)_{i \in I}$ be a Baire type of rank $\left(\xi_{i}\right)_{i \in I}$ with $\xi:=\sup _{i \in I} \xi_{i}$. For any family $\left(B_{i}\right)_{i \in I}$ of Borel subsets of $\omega^{\omega}$ of type $\left(\boldsymbol{\Gamma}_{i}\right)_{i \in I}$ there exist a strongly continuous expansion family $\mathcal{R}=\left(R^{(\eta)}\right)_{\eta \leq \xi}$ with $R^{(0)}=$ Ext, and a family $\left(S_{i}\right)_{i \in I}$ of subsets of $\operatorname{Seq}(\omega)$ satisfying:

a) For all $x \in \omega^{\omega}$ and $\eta \leq \xi$ there exists a unique $R^{(\eta)}$-branch $\hat{x}^{(\eta)} \subset \hat{x}$.

b) If $\boldsymbol{\Gamma}_{i}=\boldsymbol{\Sigma}_{1+\xi_{i}}^{0}$, then $S_{i}$ is $R^{\left(\xi_{i}\right)}$-monotone and for all $x \in \omega^{\omega}$ :

$$
x \in B_{i} \Longleftrightarrow \hat{x}^{\left(\xi_{i}\right)} \cap S_{i} \neq \emptyset .
$$

c) If $\boldsymbol{\Gamma}_{i}=\boldsymbol{\Pi}_{1+\xi_{i}}^{0}$, then $S_{i}$ is $R^{\left(\xi_{i}\right)}$-hereditary and for all $x \in \omega^{\omega}$ :

$$
x \in B_{i} \Longleftrightarrow \hat{x}^{\left(\xi_{i}\right)} \subset S_{i} .
$$


3.13. Remarks. a) Given any transfinite family $\mathcal{R}=\left(R^{(\eta)}\right)_{\eta \leq \xi}$ of tree relations on $\operatorname{Seq}(\omega)$, let us say that a pair $\left\langle\mathcal{R},\left(S_{i}\right)_{i \in I}\right\rangle$ is a representation of type $\left(\boldsymbol{\Gamma}_{i}\right)_{i \in I}$ for $\left(B_{i}\right)_{i \in I}$ if it satisfies properties a), b), c) of Theorem 3.12 (observe that these properties do not involve the notion of distinguished subrelation). The existence of such a representation (even without any distinction property) is a totally nontrivial fact. But the additional information that one can impose on $\mathcal{R}$ to be a resolution family will be crucial for the proof of Theorem 3.10 in Section 9 . Indeed as one can reasonably expect this proof will be by induction, and the only way we know to achieve it is by ensuring at each step this stronger property.

b) Let us also point out that the family $\mathcal{R}$ constructed in the proof of Theorem 3.10 will be defined by explicit recursive formulas, and it will be clear that $\mathcal{R}$ can then be taken in any admissible fragment of $L$ containing a code for the family $\left(B_{i}, \boldsymbol{\Gamma}_{i}\right)_{i \in I}$. In particular if $\left(B_{i}, \boldsymbol{\Gamma}_{i}\right)_{i \in I}$ has a code in $\Delta_{1}^{1}(\alpha)$, then we can choose $\left\langle\mathcal{R},\left(S_{i}\right)_{i \in I}\right\rangle$ with a code in $\Delta_{1}^{1}(\alpha)$ too and we shall then say that $\left\langle\mathcal{R},\left(S_{i}\right)_{i \in I}\right\rangle$ is a $\Delta_{1}^{1}(\alpha)$-representation of type $\left(\boldsymbol{\Gamma}_{i}\right)_{i \in I}$ for $\left(B_{i}\right)_{i \in I}$.

The existence of such representations will be one of the basic ingredients of the proof of the Dichotomy Theorem in Section 7 But this is only part of what we will need for this proof, which will also make use of another general property of resolution families that we will develop in the following two sections.

\section{Resolution OF QUASi-STRATEgies}

Roughly speaking one can view a resolution family $\left(R^{(\eta)}\right)_{\eta \leq \xi}$ as a family of "operators" which transform $R^{(0)}$-branches into $R^{(\eta)}$-branches via the following formulas (see Proposition 3.7):

$$
\begin{cases}\sigma^{(\eta+1)}=\left\{s \in \sigma^{(\eta)}: \forall t\left(s R^{(\eta)} t \Longrightarrow s R^{(\eta+1)} t\right)\right\} & \text { if } \eta<\xi \\ \sigma^{(\lambda)}=\bigcap_{\eta<\lambda} \sigma^{(\eta)} & \text { if } \lambda \leq \xi \text { is limit. }\end{cases}
$$

The basic idea is then to enlarge the domain of these operators and consider these formulas for arbitrary subsets $\sigma$ of $\operatorname{Dom}\left(R^{(0)}\right)$. More particularly we shall consider the case where $\operatorname{Dom}\left(R^{(0)}\right)$ is the set of all positions for some player in an arbitrary game and $\sigma$ is a quasi-strategy for this player. Observe that in this procedure the starting resolution family $\left(R^{(\eta)}\right)_{\eta \leq \xi}$ is not necessarily associated to some family $\left(B_{i}\right)_{i \in \omega}$ of Borel sets, and in this respect the results of this section are totally independent from the Representation Theorem whose only role will be to provide resolution families.

The notions discussed in this section are extracted from Section III.1 of [1] and our goal here is to strengthen (under some additional assumption) the conclusion of Theorem III-1.7.

4.1. General terminology and notation. To avoid any ambiguity we recall the definitions of some basic notions concerning games.

A game $\Gamma$ on $\omega$ is simply a tree on $\omega$. An element $u$ of $\Gamma$ will then be called a position: if $|u|$ is odd (even), we shall say that $u$ is a I-position (II-position), and if $u$ is nonempty we set:

$$
u^{*}=u_{|u|-1} .
$$

Also given any set $\Lambda \subset \operatorname{Seq}(\omega)$ we set:

$$
\Lambda_{\mathrm{I}}=\{u \in \Lambda: u \text { is a I-position }\} \quad \text { and } \quad \Lambda_{\mathrm{II}}=\{u \in \Lambda: u \text { is a II-position }\} .
$$


In the sequel, quasi-strategies will always be relative to Player $I$. We recall that a quasi-strategy in the game $\Gamma$ is a subtree $\Sigma$ of $\Gamma$ satisfying:

For any $u \in \Sigma_{\mathrm{I}}$ and $v \in \Gamma_{\mathrm{II}}$ such that $u=v^{*}$ there exists $w \in \Sigma_{\mathrm{I}}$ such that $v=w^{*}$.

We shall say that a tree $\Sigma$ is a quasi-strategy (without any reference to some game) if $\Sigma$ is a quasi-strategy in the game $\Sigma$, that is, if $\Sigma$ has no terminal element of even length.

Before going further let us make it clear that our main concern here is not game determinacy, but the study of quasi-strategies as "large sets". In particular strategies are irrelevant in this study. The reason for this is that we shall define operators on spaces of quasi-strategies. Of course one can always apply such an operator to a strategy, but then the result will still be a quasi-strategy and trying to replace it by a strategy, besides being not natural in this context, would complicate very seriously the statements.

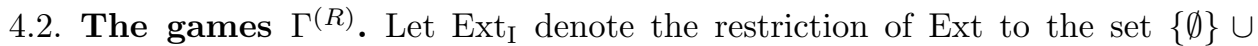
$(\operatorname{Seq}(\omega))_{\mathrm{I}}$ and let $R$ be a tree relation such that $R \subset \operatorname{Ext}_{\mathrm{I}}$.

Then given any set $\Gamma \subset \operatorname{Seq}(\omega)$ we consider the game $\Gamma^{(R)}$ on $\operatorname{Seq}(\omega)$ in which at each of their moves: Player I chooses some $u_{2 n} \in \Gamma_{\mathrm{I}}$ and Player II chooses some $u_{2 n+1} \in \Gamma_{\text {II }}$ satisfying for all $n \geq 0$ :

1) $u_{2 n} \prec u_{2 n+1} \prec u_{2 n+2}$,

2) $u_{2 n+1}^{*}=u_{2 n}$,

3) $u_{2 n-2}$ is the $R$-predecessor of $u_{2 n}$,

where by convention $u_{-2}=\emptyset$.

So at his first move in the game $\Gamma^{(R)}$, Player I chooses some $u_{0} \in \Gamma_{\mathrm{I}}$ of arbitrary length but of $R$-height 1 (clause 3 ). Then Player II chooses some $u_{1} \in \Gamma_{\text {II }}$ extending $u_{0}$ by one move (in $G$ ). Then again Player I chooses some $u_{2} \in \Gamma_{\mathrm{I}}$ of arbitrary length and which is an $R$-successor of $u_{0}$ (recall that the domain of $R$ is the set of all Ipositions plus $u_{-2}=\emptyset$ ); hence $u_{2}$ is of $R$-height 2 . Thus the successive moves $\left(u_{0}, u_{2}, \ldots, u_{2 n}\right)$ of Player I in the game $\Gamma^{(R)}$ constitute an $R$-branch.

Formally speaking $\Gamma^{(R)} \subset \operatorname{Seq}(\operatorname{Seq}(\omega))$. Notice however that any nonempty position $\bar{u}=\left(u_{0}, u_{1}, \ldots, u_{n}\right)$ in $\Gamma^{(R)}$ is entirely determined by the last move $u_{n} \in \Gamma$; hence the correspondence $\Gamma \mapsto \Gamma^{(R)}$ is one-to-one and moreover:

$$
\bar{u} \in\left(\Gamma^{(R)}\right)_{\mathrm{I}} \Longleftrightarrow u_{n} \in \Gamma_{\mathrm{I}}
$$

We recall that $\Gamma$ is an arbitrary subset of $\operatorname{Seq}(\omega)$. But when $\Gamma$ is a tree, then one can consider $\Gamma$ as a game (on $\omega$ ) and in this case the significance of the game $\Gamma^{(R)}$ is quite intuitive: one can view a run in $\Gamma^{(R)}$ as a run in $\Gamma$ in which Player $\mathrm{I}$ is allowed to choose (under some restrictions) some of Player II's moves while Player II is playing by simple moves as in $\Gamma$.

Definition 4.3. Let $R \subset$ Ext $_{\mathrm{I}}$ be a tree relation. We shall say that $\Sigma \subset \Gamma$ is $R$-strategical (implicitly for Player I) in $\Gamma$ if:

a) For any $u \in \Sigma_{\mathrm{I}}$ if $\emptyset \prec u^{\prime} R u$, then $u^{\prime} \in \Sigma_{\mathrm{I}}$.

b) For any $u \in \Sigma_{\text {I }}$ and $v \in \Gamma_{\text {II }}$ such that $u=v^{*}$ there exists $w \in \Sigma_{\text {I }}$ such that $u \prec v \prec w$ and $u$ is the $R$-predecessor of $w$. 


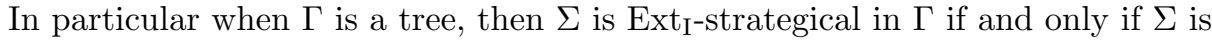
a quasi-strategy in the game $\Gamma$. Also by merely inspecting the definitions one can easily check the following:

Proposition 4.4. $\Sigma$ is R-strategical in $\Gamma$ if and only if $\Sigma^{<\omega} \cap \Gamma^{(R)}$ is a quasistrategy in the game $\Gamma^{(R)}$.

4.5. Remark. One can identify any element $\sigma \in\left[\operatorname{Ext}_{\mathrm{I}}\right]$ to some $x \in \omega^{\omega}$ but also to the trivial game in which at his $(n+1)^{\text {th }}$ move Player I has to play $x(n)$ while Player II has always to play " 0 ". Under the latter identification, $\tau \subset \sigma$ is $R$-strategical in $\sigma$ if and only if $\tau$ is an $R$-branch. Thus the notion of an $R$-strategical set is a generalization of the notion of an $R$-branch.

In particular if $\left(R^{(\eta)}\right)_{\eta \leq \xi}$ is an expansion family we shall canonically associate to any $R^{(0)}$-strategical set $\Sigma$ and any $\eta \leq \xi$ an $R^{(\eta)}$-strategical set $\Sigma^{(\eta)}$, and the mapping $\Sigma \mapsto \Sigma^{(\eta)}$ will be a generalization of the mapping $\pi_{\eta}^{-1}: \sigma \mapsto \sigma^{(\eta)}$ (where $\sigma^{(\eta)}$ denotes the unique $R^{(\eta)}$-infinite branch contained in the $R^{(0)}$-infinite branch $\sigma)$. In fact $\Sigma^{(\eta)}$ will be defined essentially by copying the formulas which compute $\sigma^{(\eta)}$ from $\sigma$ (see Proposition 3.9) and as mentioned above this latter result will actually appear as a particular case of Theorem 4.9 below.

Definition 4.6. Let $\mathcal{R}=\left(R^{(\eta)}\right)_{\eta \leq \xi}$ be a resolution family with $R^{(0)} \subset \operatorname{Ext}_{\mathrm{I}}$. For any $\Sigma \subset \operatorname{Seq}(\omega)$ we define inductively the family $\left(\Sigma^{(\eta)}\right)_{\eta \leq \xi}$ by:

$$
\begin{aligned}
& \Sigma^{(0)}=\Sigma, \\
& \left\{\Sigma_{\mathrm{I}}^{(\eta+1)}=\left\{u \in \Sigma_{\mathrm{I}}^{(\eta)}: \forall v, w \in \Sigma_{\mathrm{I}}^{(\eta)}\left(\left(v R^{(\eta+1)} u \text { and } v R^{(\eta)} w\right) \Longrightarrow v R^{(\eta+1)} w\right)\right\}\right. \\
& \left\{\Sigma_{\mathrm{II}}^{(\eta+1)}=\left\{u \in \Sigma_{\mathrm{II}}^{(\eta)}: u^{*} \in \Sigma_{\mathrm{I}}^{(\eta+1)}\right\},\right. \\
& \Sigma^{(\lambda)}=\bigcap_{\zeta<\lambda} \Sigma^{(\zeta)} \quad \text { if } \lambda \text { is limit. }
\end{aligned}
$$

Finally let:

$$
\Phi_{\mathcal{R}}(\Sigma)=\Sigma^{(\xi)}
$$

denote the last element of the family $\mathcal{R}$.

Lemma 4.7. For all $v, w \in \Sigma^{(\eta)}$ if $v R^{(0)} w$, then $v R^{(\eta)} w$.

Proof. By induction on $\eta$. For $\eta=0$ the result is trivial and the inductive step in the limit case is straightforward.

So suppose that the result is true for $\eta$ and consider $v, w \in \Sigma^{(\eta+1)}$ such that $v R^{(0)} w$. Since $R^{(0)} \subset$ Ext $_{\mathrm{I}}$, then necessarily $v, w \in \Sigma_{\mathrm{I}}^{(\eta+1)} \subset \Sigma^{(\eta)}$ and applying the inductive hypothesis we get that $v R^{(\eta)} w$, and it follows then from the very definition of $\Sigma_{\mathrm{I}}^{(\eta+1)}$ that $v R^{(\eta+1)} w$.

4.8. Remark. The formula defining $\Sigma^{(\eta+1)}$ from $\Sigma^{(\eta)}$ is slightly more complicated than the one defining $\sigma^{(\eta+1)}$ from $\sigma^{(\eta)}$ in Proposition 3.7. since it involves two universal quantifications (on $v$ and $w$ ). In fact the role of the universal quantification on $v$ is to ensure that the set $\Sigma^{(\eta+1)}$ is $R^{(\eta+1)}$ hereditary. However using the distinction of $R^{(\eta+1)}$ in $R^{(\eta)}$, one can prove that if $\Sigma^{(\eta)}$ is linearly ordered by $R^{(\eta)}$, then in this case the two formulas are equivalent. The following basic result is proved in [1]:

Theorem 4.9. For any expansion family $\mathcal{R}=\left(R^{(\eta)}\right)_{\eta \leq \xi}$ with $R^{(0)} \subset \operatorname{Ext}_{I}$, if $\Sigma \subset \operatorname{Seq}(\omega)$ is $R^{(0)}$-strategical, then $\Phi_{\mathcal{R}}(\Sigma)$ is $R^{(\xi)}$-strategical in $\Sigma$. 
This is essentially part 1) of Theorem III-1.7 in [1]. Actually this latter result is stated as an existential result, but the reader can easily check from the first lines of the proof that the mapping $\Phi$ considered there is exactly what we call $\Phi_{\mathcal{R}}$ here. One should also observe that the additional "regularity" assumption in Theorem III-1.7 is not used in the proof of this part.

We now investigate the descriptive complexity of the mapping

$$
\Phi_{\mathcal{R}}: \mathcal{P}(\operatorname{Seq}(\omega)) \rightarrow \mathcal{P}(\operatorname{Seq}(\omega))
$$

where the space $\mathcal{P}(\operatorname{Seq}(\omega))$ is identified with the space $2^{\operatorname{Seq}(\omega)}$ endowed with the product of the discrete topology on $\operatorname{Seq}(\omega)$. In fact it follows from (the proof of) the second part of Theorem III-1.7 in [1] that:

a) $\Phi_{\mathcal{R}}$ is of class $\xi+1$.

b) If $\xi$ is limit and $\mathcal{R}$ is strongly continuous at $\xi$, then $\Phi_{\mathcal{R}}$ is of class $\xi$.

But as we shall see next, part a) can be improved if we strengthen the hypothesis on $\mathcal{R}$. We recall (see Definition 3.8) that "strongly continuous" means "strongly continuous at any limit level".

Theorem 4.10. If $\mathcal{R}=\left(R^{(\eta)}\right)_{\eta \leq \xi}$ is a strongly continuous resolution family with $R^{(0)} \subset \operatorname{Ext}_{I}$, then $\Phi_{\mathcal{R}}$ is of class $\xi$.

Proof. For any $\Sigma \subset \operatorname{Seq}(\omega)$ let $\left(\Sigma^{(\eta)}\right)_{\eta \leq \xi}$ be as in Definition 4.6 and set:

$$
\mathcal{S}_{u}^{(\eta)}=\left\{\Sigma: u \in \Sigma^{(\eta)}\right\}
$$

We shall prove by induction on $\eta$ that for all $u \in \operatorname{Seq}(\omega)$ the set $\mathcal{S}_{u}^{(\eta)}$ is $\boldsymbol{\Delta}_{1+\eta}^{0}$.

For $\eta=0, \mathcal{S}_{u}^{(0)}$ is a basic clopen set of the space $\mathcal{P}(\operatorname{Seq}(\omega))$ and the result is trivial. We now prove the inductive step.

- The successor case: Suppose that for all $u$ the set $\mathcal{S}_{u}^{(\eta)}$ is $\boldsymbol{\Delta}_{1+\eta}^{0}$ and observe that from Definition 4.6 we have:

$$
\begin{aligned}
u \in \Sigma^{(\eta+1)} \Longleftrightarrow u \in \Sigma_{\mathrm{I}}^{(\eta+1)} \text { or }\left(u^{*} \in \Sigma_{\mathrm{I}}^{(\eta+1)} \text { and } u \in \Sigma^{(\eta)}\right), \\
u \in \Sigma_{\mathrm{I}}^{(\eta+1)} \Longleftrightarrow\left\{\begin{array}{l}
u \in \Sigma_{\mathrm{I}}^{(\eta)} \text { and } \forall v, w \quad\left(v \notin \Sigma_{\mathrm{I}}^{(\eta)} \text { or } w \notin \Sigma_{\mathrm{I}}^{(\eta)}\right. \\
\text { or } \left.\neg\left(v R^{(\eta+1)} u\right) \text { or } \neg\left(v R^{(\eta)} w\right) \text { or } v R^{(\eta+1)} w\right) .
\end{array}\right.
\end{aligned}
$$

Then applying the induction hypothesis one easily sees that the set $\mathcal{S}_{u}^{(\eta+1)}$ is then $\boldsymbol{\Pi}_{1+\eta}^{0}$ hence $\boldsymbol{\Delta}_{1+\eta+1}^{0}$.

- The limit case: Suppose now that $\lambda$ is a limit ordinal and that for all $u$ and all $\zeta<\lambda$ the set $\mathcal{S}_{u}^{(\zeta)}$ is $\Delta_{1+\zeta}^{0}$. Fix $u \in \operatorname{Seq}(\omega)$; since the resolution family $\left(R^{(\eta)}\right)_{\eta \leq \xi}$ is strongly continuous we can then fix $\eta<\lambda$ such that for any $v \preceq u$ we have:

$$
\forall w\left(v R^{(\eta)} w \Rightarrow v R^{(\lambda)} w\right)
$$

Fact 1. $\mathcal{S}_{u}^{(\lambda)}=\mathcal{S}_{u}^{(\eta)}$

Proof. The inclusion from left to right is obvious. Conversely suppose that $\Sigma \in \mathcal{S}_{u}^{(\eta)}$; we shall prove by induction on $\zeta<\lambda$ that $\Sigma \in \mathcal{S}_{u}^{(\zeta)}$ : For $\zeta=0$ this is obvious since $\Sigma \in \mathcal{S}_{u}^{(\eta)} \subset \mathcal{S}_{u}^{(0)}$. So suppose that $\Sigma \in \mathcal{S}_{u}^{\left(\zeta^{\prime}\right)}$ for all $\zeta^{\prime}<\zeta$; we have to prove that $u \in \Sigma^{(\zeta)}$ :

- If $\zeta$ is limit this follows directly from the definition of $\Sigma^{(\zeta)}$ in the limit case. 
- If $\zeta$ is successor, set $\zeta=\zeta^{*}+1$ and consider any $v, w \in \Sigma_{I}^{\left(\zeta^{*}\right)}$ such that $v R^{(\zeta)} u$ and $v R^{\left(\zeta^{*}\right)} w$. Then $v \preceq u$ and $v R^{(\eta)} w$; hence by the choice of $\eta$ we also have $v R^{(\lambda)} w$ and a fortiori $v R^{\left(\zeta^{*}+1\right)} w$. This proves that $u \in \Sigma^{\left(\zeta^{*}+1\right)}=\Sigma^{(\zeta)}$.

Since $\mathcal{S}_{u}^{(\lambda)}=\mathcal{S}_{u}^{(\eta)}$, then by the induction hypothesis $\mathcal{S}_{u}^{(\lambda)} \in \boldsymbol{\Delta}_{1+\eta}^{0} \subset \boldsymbol{\Delta}_{1+\lambda}^{0}$, which finishes the proof of Theorem 4.10 in the limit case.

\section{LOCAL RESOLUTION OF QUASI-STRATEGIES}

To analyze a game $\Gamma$ (here on $\omega$ ) it is quite common to consider its "localizations", that is, the games $\Gamma_{u}=\{v \in \operatorname{Seq}(\omega): u \frown v \in \Gamma\}$. But this general procedure is not adapted to our setting since we want the localization of a game $\Gamma^{(R)}$ to be itself of the same type. In fact this localization will be defined as the game $\Gamma^{\left(R_{u}\right)}$ where $R_{u}$ will be the "localization to $u$ " of the tree relation $R$. Practically all results of this section follow very simply either from the definitions or from the results of the previous section, and we shall leave some verifications to the reader. However this simplicity should not hide the fundamental fact lying behind all these results and which is the preservation of the notion of distinction by localization. We recall that $\operatorname{Dom}\left(\operatorname{Ext}_{\mathrm{I}}\right)=\{\emptyset\} \cup(\operatorname{Seq}(\omega))_{\mathrm{I}}$.

Definition 5.1. Let $R$ be a tree relation such that $R \subset \operatorname{Ext}_{\mathrm{I}}$ and $\Sigma \subset \operatorname{Seq}(\omega)$. Then for any $u \in \operatorname{Dom}\left(\operatorname{Ext}_{\mathrm{I}}\right)$ we define the set $\Sigma_{u} \subset \Sigma$ and the relation $R_{u} \subset R$ by:

$$
\begin{aligned}
& \Sigma_{u}=\{v \in \Sigma: u \preceq v\}, \\
& v R_{u} w \Longleftrightarrow(v=\emptyset \text { or } u \preceq v R w) .
\end{aligned}
$$

The following propositions follow readily from the definitions.

Proposition 5.2. Let $R$ be a tree relation such that $R \subset \operatorname{Ext}_{\mathrm{I}}$ and $\Sigma \subset \operatorname{Seq}(\omega)$. Then for any $u \in \operatorname{Dom}\left(\operatorname{Ext}_{\mathrm{I}}\right)$ :

a) $R_{u} \subset R$ is a tree relation.

b) $\left[R_{u}\right]=\left\{\sigma_{u}: \sigma \in[R]\right.$ and $\sigma_{u}$ is infinite $\}$.

c) If $\Sigma$ is $R$-strategical and $u \in \Sigma$, then $\Sigma_{u}$ is $R_{u}$-strategical.

Proposition 5.3. If $\mathcal{R}=\left(R^{(\eta)}\right)_{\eta \leq \xi}$ is a (strongly continuous) resolution (expansion) family, then $\mathcal{R}_{u}=\left(R_{u}^{(\eta)}\right)_{\eta \leq \xi}$ is a (strongly continuous) resolution (expansion) family too.

From now on we fix a resolution family $\mathcal{R}=\left(R^{(\eta)}\right)_{\eta \leq \xi}$ such that $R^{(0)} \subset \operatorname{Ext}_{I}$.

5.4. The mappings $\Phi^{\tau}$. Our goal now is to define for any $\tau$ in some large tree $\mathcal{T}$, a mapping

$$
\Phi^{\tau}: \mathcal{P}(\operatorname{Seq}(\omega)) \rightarrow \mathcal{P}(\operatorname{Seq}(\omega))
$$

which generalizes the mapping $\Phi_{\mathcal{R}}$ associated to $\mathcal{R}$ (see Definition 4.6).

The mapping $\Phi^{\tau}$ will be essentially a composition of mappings of the form $\Phi_{\mathcal{R}^{\prime}}$ where each $\mathcal{R}^{\prime}$ is a resolution family derived from $\mathcal{R}$. Since the precise definitions of $\mathcal{T}$ and $\Phi^{\tau}$ are rather tedious we shall first make them explicit in an informal way.

a) Starting from the resolution family $\mathcal{R}=\left(R^{(\eta)}\right)_{\eta \leq \xi}$ we can construct new resolution families as follows: For any $\eta \leq \eta^{\prime} \leq \xi$ and any $u \in \operatorname{Dom}\left(\operatorname{Ext}_{\mathrm{I}}\right)$ we can 
first consider the resolution family

$$
\mathcal{R}^{\left(\eta, \eta^{\prime}\right)}:=\left(R^{(\eta+\zeta)}\right)_{\zeta \leq \eta^{\prime}-\eta} \approx\left(R^{(\zeta)}\right)_{\eta \leq \zeta \leq \eta^{\prime}} .
$$

Then by Proposition 5.3 ,

$$
\mathcal{R}_{u}^{\left(\eta, \eta^{\prime}\right)}:=\left(\mathcal{R}^{\left(\eta, \eta^{\prime}\right)}\right)_{u}=\left(\mathcal{R}_{u}\right)^{\left(\eta, \eta^{\prime}\right)}
$$

is also a resolution family.

b) Fix $\Sigma \subset \operatorname{Seq}(\omega)$. For any $\eta_{0} \leq \xi$, let $\mathcal{R}_{0}=\left(R^{(\eta)}\right)_{0 \leq \eta \leq \eta_{0}}$ and define

$$
\Sigma^{\left(\eta_{0}\right)}:=\Phi_{\mathcal{R}_{0}}(\Sigma) \subset \Sigma .
$$

Then given any $v_{0} \in \operatorname{Dom}\left(\operatorname{Ext}_{I}\right)$ we can define

$$
\Sigma^{\left(\eta_{0}, v_{0}\right)}:=\left(\Sigma^{\left(\eta_{0}\right)}\right)_{v_{0}} \subset \Sigma^{\left(\eta_{0}\right)} \cap \Sigma_{v_{0}} \subset \Sigma_{v_{0}} .
$$

Now given any $\eta_{1} \geq \eta_{0}$ let $\mathcal{R}_{1}=\mathcal{R}_{v_{0}}^{\left(\eta_{0}, \eta_{1}\right)}$ and define

$$
\Sigma^{\left(\eta_{0}, v_{0}, \eta_{1}\right)}:=\Phi_{\mathcal{R}_{1}}\left(\Sigma^{\left(\eta_{0}, v_{0}\right)}\right) \subset \Sigma^{\left(\eta_{0}, v_{0}\right)} \subset \Sigma_{v_{0}},
$$

and again given any $v_{1} \succeq v_{0}$ we can define

$$
\Sigma^{\left(\eta_{0}, v_{0}, \eta_{1}, v_{1}\right)}:=\left(\Sigma^{\left(\eta_{0}, v_{0}, \eta_{1}\right)}\right)_{v_{1}} \subset \Sigma^{\left(\eta_{0}, v_{0}, \eta_{1}\right)} \cap \Sigma_{v_{1}} \subset \Sigma_{v_{1}}
$$

and so on.

c) Observe that if $\Sigma$ is $R^{(0)}$-strategical, then by Theorem 4.9 , $\Sigma^{\left(\eta_{0}\right)}$ is $R^{\left(\eta_{0}\right)_{-}}$ strategical. If moreover $v_{0} \in \Sigma^{\left(\eta_{0}\right)}$, then by Proposition [5.2, $\Sigma^{\left(\eta_{0}, v_{0}\right)}$ is $R_{v_{0}}^{\left(\eta_{0}\right)}$ strategical and obviously $v_{0} \in \Sigma^{\left(\eta_{0}, v_{0}\right)}$. Then again by Theorem 4.9 . $\Sigma^{\left(\eta_{0}, v_{0}, \eta_{1}\right)}$ is $R_{v_{0}}^{\left(\eta_{1}\right)}$-strategical but $v_{0}$ might be no more in $\Sigma^{\left(\eta_{0}, v_{0}, \eta_{1}\right)}$. If moreover $v_{1} \in \Sigma^{\left(\eta_{0}, v_{0}, \eta_{1}\right)}$, then $\Sigma^{\left(\eta_{0}, v_{0}, \eta_{1}, v_{1}\right)}$ is $R_{v_{1}}^{\left(\eta_{1}\right)}$-strategical and $v_{1} \in \Sigma^{\left(\eta_{0}, v_{0}, \eta_{1}, v_{1}\right)}$.

d) We now proceed to a precise formal definition. Let $\mathcal{T}$ be the tree of all "alternate" sequences

$$
\tau=\left(\eta_{0}, v_{0}, \eta_{1}, v_{1}, \ldots\right),
$$

where $\eta_{0} \leq \eta_{1} \leq \ldots$ is a nondecreasing sequence of ordinals $\leq \xi$, and $v_{0} \preceq v_{1} \preceq \ldots$ is a nondecreasing sequence of positions in $\operatorname{Dom}\left(\operatorname{Ext}_{I}\right)$.

For all $\tau \in \mathcal{T}$ we define

$$
\ell(\tau)=\left(\ell_{0}(\tau), \ell_{1}(\tau)\right) \in \omega_{1} \times \operatorname{Seq}(\omega)
$$

by

$$
\begin{cases}\ell_{0}(\tau)=\max \{\tau(k): k \text { even }<|\tau|\} & \text { or } 0 \text { if } \tau=\emptyset \\ \ell_{1}(\tau)=\bigcup\{\tau(k): k \text { odd }<|\tau|\} & \text { or } \emptyset \text { if }|\tau| \leq 1\end{cases}
$$

in particular $\ell(\emptyset)=(0, \emptyset)$. If $\tau \neq \emptyset$ we denote by $\tau^{*}$ its predecessor in $\mathcal{T}$.

We now define, by induction on $|\tau|$, for $\tau \in \mathcal{T}$ the mapping $\Phi^{\tau}$. We first set:

$$
\Phi^{\emptyset}=\mathrm{Id} .
$$

Suppose that $\Phi^{\tau}$ is already defined for some $\tau \in \mathcal{T}$ of even length and set $\ell(\tau)=$ $(\eta, v)$. Then for all $\eta^{\prime} \geq \eta$ and all $v^{\prime} \succeq v$ we define for $\Sigma \subset \operatorname{Seq}(\omega)$ :

$$
\Phi^{\tau^{\smile}\left\langle\eta^{\prime}\right\rangle}(\Sigma)=\Phi_{\mathcal{R}_{v}^{\left(\eta, \eta^{\prime}\right)}}\left(\Phi^{\tau}(\Sigma)\right) \quad \text { and } \quad \Phi^{\tau^{\smile}\left\langle\left(\eta^{\prime}, v^{\prime}\right)\right\rangle}(\Sigma)=\left(\Phi^{\tau^{\smile}\left\langle\eta^{\prime}\right\rangle}(\Sigma)\right)_{v^{\prime}} .
$$

We also define by induction on $|\tau|$ a set $\mathcal{S}^{\tau} \subset \mathcal{P}(\operatorname{Seq}(\omega))$ as follows:

$$
\mathcal{S}^{\emptyset}=\left\{\Sigma \subset \operatorname{Seq}(\omega): \Sigma \text { is } R^{(0)} \text {-strategical }\right\}
$$


and if $\tau \neq \emptyset$ :

$$
\mathcal{S}^{\tau}= \begin{cases}\left\{\Sigma \in \mathcal{S}^{\tau^{*}}: u \in \Phi^{\tau^{*}}(\Sigma)\right\} & \text { if }|\tau| \text { is even and } \ell_{1}(\tau)=u, \\ \mathcal{S}^{\tau^{*}} & \text { if }|\tau| \text { is odd. }\end{cases}
$$

(For the motivation of this definition see the observations in c).)

Finally let us point out that for the applications we shall only deal with sequences $\tau$ of even length and the consideration of sequences of odd length is only needed for the inductive definition of $\Phi^{\tau}$. For this reason some of the properties are either trivial or nonsignificant in the odd case.

Theorem 5.5. Let $\mathcal{R}$ be an expansion family and $\tau \in \mathcal{T}$ with $\ell(\tau)=(\eta, u)$. If $\Sigma \in \mathcal{S}^{\tau}$ is $R^{(0)}$-strategical, then

a) $\Phi^{\tau}(\Sigma)$ is $R_{u}^{(\eta)}$-strategical.

b) If $|\tau|$ is even, then $u \in \Phi^{\tau}(\Sigma)$.

c) For all $v, w \in \Phi^{\tau}(\Sigma)$, if $v R^{(0)} w$, then $v R^{(\eta)} w$.

d) For all $w \in \Phi^{\tau}(\Sigma)$ if $u \preceq v R^{(\eta)} w$, then $v \in \Phi^{\tau}(\Sigma)$.

Proof. Observe that d) follows from a) since an $R_{u}^{(\eta)}$-strategical set is $R_{u}^{(\eta)}$-hereditary. We now prove a), b), c) simultaneously by induction on $|\tau|$.

For $\tau=\emptyset$ the result is trivial since $\Phi^{\emptyset}(\Sigma)=\Sigma$. Suppose that the result is true for $\tau^{*}$ and set $\ell\left(\tau^{*}\right)=\left(\eta^{*}, u^{*}\right)$. Then by the induction hypothesis $\Sigma^{*}:=\Phi^{\tau^{*}}(\Sigma)$ is $R_{u^{*}}^{\left(\eta^{*}\right)}$-strategical.

- If $|\tau|$ is even, then $\eta=\eta^{*}$ and $\Phi^{\tau}(\Sigma)=\Sigma_{u}^{*}$; and since $\Sigma \in \mathcal{S}^{\tau}$, then $u \in \Sigma^{*}$. Hence by Proposition $5.2 \mathrm{c}), \Phi^{\tau}(\Sigma)$ is $R^{\prime}$-strategical with $R^{\prime}=\left(R_{u^{*}}^{\left(\eta^{*}\right)}\right)_{u}=\left(R_{u^{*}}^{(\eta)}\right)_{u}=$ $R_{u}^{(\eta)}$, which proves a). Moreover since $u \in \Sigma^{*}$, then obviously $u \in \Sigma^{*} \in \Phi^{\tau}(\Sigma)$, which proves b). Finally c) follows from the induction hypothesis since $\Phi^{\tau}(\Sigma) \subset$ $\Phi^{\tau^{*}}(\Sigma)$ and $\eta=\eta^{*}$.

— If $|\tau|$ is odd, then $u=u^{*}$ and $\Phi^{\tau}(\Sigma)=\Phi_{\mathcal{R}_{u}^{\left(\eta^{*}, \eta\right)}}\left(\Sigma^{*}\right)$. Then by Theorem 4.9, $\Phi^{\tau}(\Sigma)$ is $R^{\prime \prime}$-strategical with $R^{\prime \prime}=\left(R_{u}^{(\eta)}\right)_{u}^{\left(\eta-\eta^{*}\right)}=R_{u}^{(\eta)}$, which proves a). In this case there is nothing to prove for b), and c) follows from Lemma 4.7 applied to $\mathcal{R}_{u}^{\left(\eta^{*}, \eta\right)}$.

Theorem 5.6. Let $\mathcal{R}$ be a resolution family and $\tau \in \mathcal{T}$ with $\ell_{0}(\tau)=\eta$. If $\mathcal{R}$ is strongly continuous, then the mapping $\Phi^{\tau}$ is of class $\eta$.

Proof. By induction on $|\tau|$ : for $\tau=\emptyset$ the result is trivial since $\Phi^{\emptyset}=$ Id. Suppose that the result is true for $\tau^{*}$, and set $\ell(\tau)=(\eta, u)$ and $\ell\left(\tau^{*}\right)=\left(\eta^{*}, u^{*}\right)$.

- If $|\tau|$ is even, then $\eta=\eta^{*}$ and $\Phi^{\tau}=\Psi_{u} \circ \Phi^{\tau^{*}}$ where $\Psi_{u}$ denotes the continuous mapping $\Sigma \mapsto \Sigma_{u}$. Hence $\Phi^{\tau}$ is of class $\eta$.

- If $|\tau|$ is odd, then $u=u^{*}$ and $\Phi^{\tau}=\Phi_{\mathcal{R}_{u}^{\left(\eta^{*}, \eta\right)}} \circ \Phi^{\tau^{*}}$ and by Theorem 4.10, $\Phi_{\mathcal{R}_{u}^{\left(\eta^{*}, \eta\right)}}$ is of class $\eta-\eta^{*}$. Hence $\Phi^{\tau}$ is of class $\left(\eta-\eta^{*}\right)+\eta^{*}=\eta$.

5.7. Remark. As for Theorem 3.12 one can observe that the statement of the previous results can be considered for any transfinite family $\mathcal{R}$, and the reader can check that what we will really use in the proof of the Dichotomy Theorem in Section 7 is the following (see Remark 3.13):

Any family $\left(B_{i}\right)_{i \in I}$ of Borel subsets of $\omega^{\omega}$ of type $\left(\boldsymbol{\Gamma}_{i}\right)_{i \in I}$ admits a representation $\left\langle\mathcal{R},\left(S_{i}\right)_{i \in I}\right\rangle$ of type $\left(\boldsymbol{\Gamma}_{i}\right)_{i \in I}$ such that if we identify $\mathcal{R}$ to a family of tree relations on Dom(Ext ${ }_{\mathrm{I}}$, then the conclusions of Theorems 5.5 and 5.6 hold. 
Moreover if $\left(B_{i}\right)_{i \in I}$ and $\left(\boldsymbol{\Gamma}_{i}\right)_{i \in I}$ have codes in $\Delta_{1}^{1}(\alpha)$, then $\left\langle\mathcal{R},\left(S_{i}\right)_{i \in I}\right\rangle$ can be chosen with a code in $\Delta_{1}^{1}(\alpha)$ too.

\section{Adequate Baire types}

6.1. Notation. Let $\left(\boldsymbol{\Gamma}_{i}\right)_{i \in I}$ be a Baire type of rank $\left(\xi_{i}\right)_{i \in I}$. It is most important to distinguish between the set $I^{(+)}$of all $i \in I$ such that $\boldsymbol{\Gamma}_{i}$ is additive from the set $I^{(\times)}$of all $i \in I$ such that $\boldsymbol{\Gamma}_{i}$ is multiplicative. But the situation is not symmetrical and the latter set will play a more fundamental role. Also for simplicity we set:

$$
J=I^{(\times)}=\left\{j \in I: \boldsymbol{\Gamma}_{j}=\boldsymbol{\Pi}_{\xi_{j}}^{0}\right\} .
$$

For all $i \in I$ and $\xi \in \omega_{1}$ let

$$
J_{i}:=\left\{j \in J: \boldsymbol{\Gamma}_{j} \subset \boldsymbol{\Gamma}_{i}\right\}, \quad J(\xi):=\left\{j \in J: \boldsymbol{\Gamma}_{j}=\boldsymbol{\Pi}_{\xi}^{0}\right\}
$$

and finally let

$$
\Omega:=\left\{\xi_{j}: j \in J\right\}, \quad \lambda:=\sup \Omega .
$$

We follow this notation in all the statements and proofs of this section.

Definition 6.2. We shall say that the Baire type $\left(\boldsymbol{\Gamma}_{i}\right)_{i \in I}$ is adequate if for all $i \in I$ such that $\boldsymbol{\Gamma}_{i} \neq \boldsymbol{\Sigma}_{\lambda}^{0}$ the set $J_{i}=\left\{j \in J: \boldsymbol{\Gamma}_{j} \subset \boldsymbol{\Gamma}_{i}\right\}$ is finite.

For example the Baire types $\left(\boldsymbol{\Pi}_{k+1}^{0}\right)_{k \in \omega},\left(\boldsymbol{\Pi}_{k+1}^{0}\right)_{k \in \omega}\left\langle\boldsymbol{\Sigma}_{\omega}^{0}\right\rangle$ are adequate while $\left(\boldsymbol{\Pi}_{k+1}^{0}\right) \widehat{k \in \omega}\left\langle\boldsymbol{\Pi}_{\omega}^{0}\right\rangle,\left(\boldsymbol{\Pi}_{k+1}^{0}\right)_{k \in \omega}\left\langle\boldsymbol{\Sigma}_{\omega+1}^{0}\right\rangle$ are not. The following result will give a more concrete description of an adequate Baire type.

Proposition 6.3. The Baire type $\left(\boldsymbol{\Gamma}_{i}\right)_{i \in I}$ is adequate if and only if:

- either $J$ is finite,

- or $J$ is infinite and the following three conditions are fulfilled:

a) $J(\xi)$ is finite for all $\xi$,

b) $\Omega$ is of order type $\omega$,

c) $\lambda=\sup \left\{\xi_{i}: i \in I\right\}$.

Proof. If $J$ is finite, then $\left(\boldsymbol{\Gamma}_{i}\right)_{i \in I}$ is obviously adequate. So we can assume that $J$ is infinite.

- Suppose that $\left(\boldsymbol{\Gamma}_{i}\right)_{i \in I}$ is adequate.

Firstly for all $\xi \in \omega_{1}$, if $\xi \notin \Omega$, then $J(\xi)$ is empty; if not, then $\xi=\xi_{i}$ for some $i \in J$. Hence $\boldsymbol{\Gamma}_{i}=\boldsymbol{\Pi}_{\xi_{i}}^{0} \neq \boldsymbol{\Sigma}_{\lambda}^{0}$ and so $J_{i}$ is finite, and it follows that $J(\xi) \subset J_{i}$ is finite too.

Secondly observe that since $J=\bigcup_{\xi \in \Omega} J(\xi)$ is infinite, then necessarily $\Omega$ is infinite. Moreover for all $\xi=\xi_{i} \in \Omega$ since $\boldsymbol{\Gamma}_{i}=\boldsymbol{\Pi}_{\xi_{i}}^{0} \neq \boldsymbol{\Sigma}_{\lambda}^{0}$, then the set $J_{i}$ is finite. Hence the set $\{\eta \in \Omega: \eta \leq \xi\}=\left\{\xi_{j}: j \in J_{i}\right\}$ is finite too. This proves that $\Omega$ is of order type $\omega$.

Finally suppose that $\lambda<\xi_{i}$ for some $i \in I$. Then $\boldsymbol{\Gamma}_{i} \neq \boldsymbol{\Sigma}_{\lambda}^{0}$ and $J_{i}=J$ would be infinite which is a contradiction.

- Conversely suppose that a), b), c) hold and fix $i \in I$ such that $\boldsymbol{\Gamma}_{i} \neq \boldsymbol{\Sigma}_{\lambda}^{0}$.

Observe that by $c$ ) we necessarily have $\xi_{i} \leq \lambda$. On the other hand, if we had $\xi_{i}=\lambda$, then we would necessarily have $\boldsymbol{\Gamma}_{i}=\boldsymbol{\Pi}_{\lambda}^{0}$. Hence $\xi_{i}=\lambda \in \Omega$ and so $\lambda$ would be a nonisolated element of the ordered set $\Omega$ which contradicts $b$ ). This proves that $\xi_{i}<\lambda$.

Now fix any $\eta \in \Omega$ such that $\xi_{i}<\eta<\lambda$; so $J_{i} \subset \bigcup_{\xi \in \Omega \cap[0, \eta]} J(\xi)$ and it follows then from a) and b) that $J_{i}$ is finite. 
Corollary 6.4. If the Baire type $\left(\boldsymbol{\Gamma}_{i}\right)_{i \in I}$ is adequate and no $\boldsymbol{\Gamma}_{i}$ is an additive Baire class of limit rank, then for all $i \in I$ the set $J_{i}=\left\{j \in J: \boldsymbol{\Gamma}_{j} \subset \boldsymbol{\Gamma}_{i}\right\}$ is finite.

Proof. We may suppose that the set $J$ is infinite. It follows then from condition b) of Proposition 6.3 that $\lambda:=\sup \left\{\xi_{j}: j \in J\right\}$ is a limit ordinal. Hence for all $i \in I$, $\boldsymbol{\Gamma}_{i} \neq \boldsymbol{\Sigma}_{\lambda}^{0}$ and since $\left(\boldsymbol{\Gamma}_{i}\right)_{i \in I}$ is adequate, then the set $J_{i}=\left\{j \in J: \boldsymbol{\Gamma}_{j} \subset \boldsymbol{\Gamma}_{i}\right\}$ is finite.

We will also need the following criterion.

Proposition 6.5. The Baire type $\left(\boldsymbol{\Gamma}_{i}\right)_{i \in I}$ is nonadequate if and only there exist $i_{*} \in I$ and $\xi_{*} \leq \lambda$ such that $\boldsymbol{\Gamma}_{i_{*}} \supset \Pi_{\xi_{*}}^{0}$ and $J_{*}=\left\{j \in J: \xi_{j} \leq \xi_{*}\right\}$ is infinite.

Moreover if $\left(\boldsymbol{\Gamma}_{i}\right)_{i \in I}$ has a code in $\Delta_{1}^{1}(\alpha)$, then we can choose $\xi_{*}$ with a code in $\Delta_{1}^{1}(\alpha)$.

Proof. Suppose first that the condition is fulfilled. We distinguish two cases:

- If $\boldsymbol{\Gamma}_{i_{*}} \neq \boldsymbol{\Sigma}_{\lambda}^{0}$, then for all $j \in J_{*}$ we have $\boldsymbol{\Gamma}_{j}=\boldsymbol{\Pi}_{\xi_{j}}^{0} \subset \boldsymbol{\Pi}_{\xi_{*}}^{0} \subset \boldsymbol{\Gamma}_{i_{*}}$. Hence $J_{*} \subset J_{i_{*}}$ so $J_{i_{*}}$ is infinite and $\left(\boldsymbol{\Gamma}_{i}\right)_{i \in I}$ is not adequate.

- If $\boldsymbol{\Gamma}_{i_{*}}=\boldsymbol{\Sigma}_{\lambda}^{0}$, then since $\boldsymbol{\Pi}_{\xi_{*}}^{0} \subset \boldsymbol{\Gamma}_{i_{*}}$ we necessarily have $\xi^{*}<\xi_{i_{*}}=\lambda$; but from the definition of $\lambda$ we can then find some $j_{*} \in J$ such that $\xi^{*}<\xi_{j_{*}}<\xi_{i_{*}}=\lambda$. Then $\boldsymbol{\Gamma}_{j_{*}}=\boldsymbol{\Pi}_{\xi_{j_{*}}}^{0} \neq \boldsymbol{\Sigma}_{\lambda}^{0}$ and as in the pevious case we would have $J_{*} \subset J_{j_{*}}$ so $J_{j_{*}}$ is infinite and $\left(\boldsymbol{\Gamma}_{i}\right)_{i \in I}$ is not adequate.

Conversely suppose that $\left(\boldsymbol{\Gamma}_{i}\right)_{i \in I}$ is not adequate. Then the set $J$ is necessarily infinite. Hence at least one of the three conditions a), b), c) of Proposition 6.3 fails, and we distinguish the three cases:

- If condition a) fails take $i_{*}$ such that $J\left(\xi_{i_{*}}\right)$ is infinite and set $\xi_{*}=\xi_{i_{*}}$ so that $J_{*} \supset J\left(\xi_{i_{*}}\right)$.

- If condition b) fails take $i_{*}$ such that $\xi_{i_{*}} \in \Omega$ and $\Omega_{*}:=\left\{\xi \in \Omega: \xi \leq \xi_{i_{*}}\right\}$ is infinite and set $\xi_{*}=\xi_{i_{*}}$ so that $J_{*} \supset\left\{j \in J: \xi_{j} \in \Omega_{*}\right\}$.

- If condition c) fails take $i_{*}$ such that $\lambda<\xi_{i_{*}}$ and set $\xi_{*}=\lambda$ so that $J_{*}=J$.

For the last part of the conclusion observe that each of the previous conditions (viewed as predicates on $\alpha$ ) is Borel and it is clear from the proof that one can always choose $\xi_{*}$ to be either $\xi_{i_{*}}$ or $\lambda$ which both have codes in $\Delta_{1}^{1}(\alpha)$.

\section{A DICHOTOMY FOR COANALYTIC COVERINGS}

For the notion of weak reduction of families of sets, see Definition 2.3 .

Theorem 7.1. Let $\left(\boldsymbol{\Gamma}_{i}\right)_{i \in I}$ be an adequate Baire type with code $\alpha \in \omega^{\omega}$. Then for any $\Delta_{1}^{1}(\alpha)$ family $\left(B_{i}\right)_{i \in I}$ of type $\left(\boldsymbol{\Gamma}_{i}\right)_{i \in I}$ and any $\Pi_{1}^{1}(\alpha)$ covering $\left(C_{i}\right)_{i \in I}$ at least one of the following two alternatives holds:

- either $\left(C_{i}\right)_{i \in I}$ admits a $\Delta_{1}^{1}(\alpha)$-subordinate covering of type $\left(\boldsymbol{\Gamma}_{i}\right)_{i \in I}$,

- or $\left(B_{i}\right)_{i \in I}$ is weakly reducible to $\left(\omega^{\omega} \backslash C_{i}\right)_{i \in I}$.

Proof. The reader can check that all the arguments below are uniform in $\alpha$ so for simplicity we shall assume that $\alpha$ is $\Delta_{1}^{1}$. For all $i \in I$ let $1+\xi_{i}$ be the rank of $\boldsymbol{\Gamma}_{i}$; set $\xi:=\sup _{i \in \omega} \xi_{i}$,

$$
I^{(+)}:=\left\{i \in I: \boldsymbol{\Gamma}_{i}=\boldsymbol{\Sigma}_{1+\xi_{i}}^{0}\right\} \quad \text { and } \quad I^{(\times)}:=\left\{i \in I: \boldsymbol{\Gamma}_{i}=\boldsymbol{\Pi}_{1+\xi_{i}}^{0}\right\} .
$$

First reduction step: For technical reasons we shall actually prove the theorem when the adequate Baire type $\left(\boldsymbol{\Gamma}_{i}\right)_{i \in I}$ satisfies some additional assumption, and then derive the general result from this particular case. 
Let us say that an adequate Baire type $\left(\boldsymbol{\Gamma}_{i}\right)_{i \in I}$ is regular if no $\boldsymbol{\Gamma}_{i}$ is an additive Baire class of limit rank. So suppose that the theorem is already proved for any regular adequate Baire type.

Consider a non-regular adequate Baire type $\left(\boldsymbol{\Gamma}_{i}\right)_{i \in I}$ and let $L$ be the set of all $j \in I^{(+)}$such that $\xi_{j}$ is limit. For all $j \in L$ fix a fundamental sequence $\left(\xi^{(j, n)}\right)_{n \in \omega}$ for $\xi_{j}$ (i.e. $\left.\xi_{j}=\sup _{n \in \omega} \xi^{(j, n)}\right)$; we shall also suppose that each $\xi^{(j, n)}$ is a successor ordinal. Then $L$ is clearly $\Delta_{1}^{1}$ and we can also assume that $\left(\xi^{(j, n)}\right)_{(j, n) \in L \times \omega}$ has a $\Delta_{1}^{1}$ code so we can find a $\Delta_{1}^{1}$ family $\left(B_{(j, n)}\right)_{(j, n) \in L \times \omega}$ such that $B_{j}=\bigcup_{n} B_{(j, n)}$ and $B_{(j, n)} \in \boldsymbol{\Sigma}_{\xi^{(j, n)}}^{0}$ for all $j \in L$.

Set $K=(I \backslash L)+L \times \omega$ (disjoint union). Consider the $\Delta_{1}^{1}$ Baire type $\left(\tilde{\boldsymbol{\Gamma}}_{k}\right)_{k \in K}$, the $\Delta_{1}^{1}$ family $\left(\tilde{B}_{k}\right)_{k \in K}$ and the $\Pi_{1}^{1}$ covering $\left(\tilde{C}_{k}\right)_{k \in K}$ defined by

$$
\begin{array}{llll}
\tilde{\boldsymbol{\Gamma}}_{k}=\boldsymbol{\Gamma}_{k} ; & \tilde{B}_{k}=B_{k} ; & \tilde{C}_{k}=C_{k} & \text { if } k \in I \backslash L, \\
\tilde{\boldsymbol{\Gamma}}_{k}=\Sigma_{\xi^{(j, n)}}^{0} ; & \tilde{B}_{k}=B_{(j, n)} ; & \tilde{C}_{k}=C_{j} & \text { if } k=(j, n) \in L \times \omega .
\end{array}
$$

Then $\left(\tilde{B}_{k}\right)_{k \in K}$ is clearly of Baire type $\left(\tilde{\boldsymbol{\Gamma}}_{k}\right)_{k \in K}$, which is still adequate but also obviously regular; moreover one can easily check that:

(1) If $\left(\tilde{C}_{k}\right)_{k \in K}$ admits a $\Delta_{1}^{1}$-subordinate covering $\left(\tilde{A}_{k}\right)_{k \in K}$ of type $\left(\tilde{\boldsymbol{\Gamma}}_{k}\right)_{k \in K}$ and if we define $\left(A_{i}\right)_{i \in I}$ by:

$$
\begin{cases}A_{i}=\tilde{A}_{i} & \text { if } i \in I \backslash L \\ A_{i}=\bigcup_{n \in \omega} \tilde{A}_{(i, n)} & \text { if } i \in L\end{cases}
$$

then $\left(A_{i}\right)_{i \in I}$ is a $\Delta_{1}^{1}$-subordinate covering to $\left(C_{i}\right)_{i \in I}$ of type $\left(\boldsymbol{\Gamma}_{i}\right)_{i \in I}$.

(2) If $f$ is a weak reduction of $\left(\tilde{B}_{k}\right)_{k \in K}$ to $\left(\omega^{\omega} \backslash \tilde{C}_{k}\right)_{k \in K}$, then $f$ is also a weak reduction of $\left(B_{i}\right)_{i \in I}$ to $\left(\omega^{\omega} \backslash C_{i}\right)_{i \in I}$.

From now on we suppose that the adequate Baire type $\left(\boldsymbol{\Gamma}_{i}\right)_{i \in I}$ is regular. In particular (see Corollary 6.4) for all $i \in I$ the set $\left\{j \in I^{(\times)}: \boldsymbol{\Gamma}_{j} \subset \boldsymbol{\Gamma}_{i}\right\}$ is finite.

As explained in the Introduction our plan is to define an open game $G$ such that if Player I wins, then the first alternative of Theorem 7.1 holds, and if Player II wins, then the second alternative holds.

Fix a $\Pi_{1}^{1}$ covering $\left(C_{i}\right)_{i \in \omega}$ and a $\Delta_{1}^{1}$ sequence $\left(T_{i}\right)_{i \in \omega}$ of trees on $\omega \times \omega$ such that for all $i$ the projection of the set $\left\lceil T_{i}\right\rceil$ (that we identify to a closed subset of $\left.\omega^{\omega} \times \omega^{\omega}\right)$ on the first factor is the $\Sigma_{1}^{1}$ set $\omega^{\omega} \backslash C_{i}$.

We identify any tree $T$ on $\omega \times \omega$ to a subset of $\bigcup_{n \in \omega} \omega^{n} \times \omega^{n}$. Then given an arbitrary pair $(t, \theta) \in \operatorname{Seq}(\omega) \times \operatorname{Seq}(\omega)$ we shall say that $(t, \theta)$ is compatible if $\left(t_{\left.\right|_{k}}, \theta_{\left.\right|_{k}}\right) \in T$ where $k=\operatorname{Dom}(t) \cap \operatorname{Dom}(\theta)$.

Applying Theorem 3.12 we also fix a $\Delta_{1}^{1}$-representation $\left\langle\left(R^{(\eta)}\right)_{\eta \leq \xi},\left(S_{i}\right)_{i \in I}\right\rangle$ of type $\left(\boldsymbol{\Gamma}_{i}\right)_{i \in I}$ for $\left(B_{i}\right)_{i \in I}$. We recall that $S_{i} \subset \operatorname{Seq}(\omega)$ is such that $S_{i}$ is $R^{\left(\xi_{i}\right)}$ monotone if $i \in I^{(+)}$, and $R^{\left(\xi_{i}\right)}$-hereditary if $i \in I^{(\times)}$; moreover for all $x \in \omega^{\omega}$ if $\hat{x}^{\left(\xi_{i}\right)}=\left\{x_{\left.\right|_{k_{n}}}: n \in \omega\right\}$ is the unique $R^{\left(\xi_{i}\right)}$-branch determined by $x$, then

$$
\begin{cases}\left(x \in B_{i} \Longleftrightarrow \exists n, x_{\left.\right|_{k_{n}}} \in S_{i}\right) & \text { if } i \in I^{(+)}, \\ \left(x \in B_{i} \Longleftrightarrow \forall n, x_{\left.\right|_{k_{n}}} \in S_{i}\right) & \text { if } i \in I^{(\times)} .\end{cases}
$$

Second reduction step: We shall also assume that

$$
\bigcup_{i \in I} S_{i}=\operatorname{Seq}(\omega) \text { and } I^{(\times)} \text {is infinite. }
$$


To see that this reduction is justified observe that starting from any adequate Baire type $\left(\boldsymbol{\Gamma}_{i}\right)_{i \in I}$ we can always find an infinite increasing sequence $\left(\eta_{n}\right)_{n \in \omega}$ of ordinals with $\Delta_{1}^{1}$ code such that the Baire type $\left(\boldsymbol{\Gamma}_{i}\right)_{i \in I}+\left(\boldsymbol{\Pi}_{1+\eta_{n}}^{0}\right)_{n \in \omega}$ is still adequate. Indeed:

- If $I(x)$ is finite take for $\left(\eta_{n}\right)_{n \in \omega}$ any increasing sequence beyond $\xi$.

- If $I_{(\times)}$is infinite take for $\left(\eta_{n}\right)_{n \in \omega}$ the increasing enumeration of $\left\{\xi: i \in I_{(x)}\right\}$. Since $\left(\boldsymbol{\Gamma}_{i}\right)_{i \in I}$ is regular, then obviously $\left(\boldsymbol{\Gamma}_{i}\right)_{i \in I}+\left(\boldsymbol{\Pi}_{1+\eta_{n}}^{0}\right)_{n \in \omega}$ is regular too. Then defining for all $n \in \omega: \boldsymbol{\Gamma}_{n}:=\boldsymbol{\Pi}_{1+\eta_{n}}^{0}, S_{n}:=\left\{\left.s^{(n)}\right|_{k}: k \leq\left|s^{(n)}\right|\right\}$ where $\left(s^{(n)}\right)_{n \in \omega}$ is any recursive enumeration of $\operatorname{Seq}(\omega)$, and $B_{n}=C_{n}=\emptyset$, it is clear that $\left\langle\boldsymbol{\Gamma}_{i}, S_{i}\right\rangle_{i \in I+\omega}$ is also a $\Delta_{1}^{1}$-representation of $\left(B_{i}\right)_{i \in I+\omega}$ and $\bigcup_{i \in I+\omega} S_{i}=\operatorname{Seq}(\omega)$; moreover $I_{(\times)}+\omega$ is infinite. Finally one easily checks that if the theorem holds for $\left\langle\boldsymbol{\Gamma}_{i}, S_{i}, B_{i}, C_{i}\right\rangle_{i \in I+\omega}$, then it also holds for $\left\langle\boldsymbol{\Gamma}_{i}, S_{i}, B_{i}, C_{i}\right\rangle_{i \in I}$.

$\underline{\text { Notation: For any } s \in \operatorname{Seq}(\omega) \text { let: }}$

$$
\begin{aligned}
\mu(s) & :=\min \left\{\xi_{j}: s \in S_{j}\right\}, \\
I(s) & :=\left\{i \in I: \xi_{i}=\mu(s) \text { and } s \in S_{i}\right\}, \\
J(s) & := \begin{cases}I(s) \cap I^{(+)} & \text {if this set is nonempty, } \\
I(s) & \text { if } I(s) \subset I_{(\times)} .\end{cases}
\end{aligned}
$$

To give the intuition behind the definition of $J(s)$ recall that each $S_{j}$ is a coding for the Borel set $B_{j}$, and the relation " $s \in S_{j}$ " should be interpreted as an "indication" that $s$ "might" be the beginning of a real $x$ in $B_{j}$. Our aim is to define a "guess" procedure which will determine some $j$ such that $x \in B_{j}$. Thus in defining $\eta=\mu(s)$ we are looking for such a $B_{j}$ of minimal Borel rank, and among all possible $B_{j}$ of this minimal rank $1+\eta$, the definition of $J(s)$ gives the priority to $\boldsymbol{\Sigma}_{1+\eta}^{0}$ sets in opposition to $\boldsymbol{\Pi}_{1+\eta}^{0}$ sets. The last step will be to choose some particular $j \in J(s)$; and this will be achieved through the open game that we introduce now.

The game $G$ : A run in the game $G$ will go as follows:

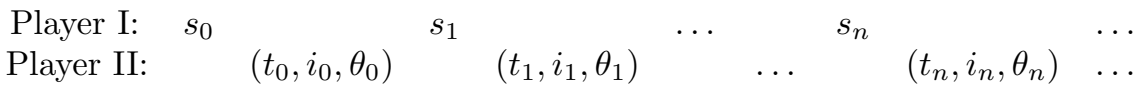

with $s_{n} \in 2^{n+1}$ and $\left(t_{n}, i_{n}, \theta_{n}\right) \in 2^{n+1} \times I \times \operatorname{Seq}(\omega)$ and satisfying the following rules:

$\left(\mathrm{R}_{0}\right): s_{n-1} \prec s_{n}$ and $t_{n-1} \prec t_{n}$,

$\left(\mathrm{R}_{1}\right): i_{n} \in J\left(s_{n}\right)$ (hence $\xi_{i_{n}}=\mu\left(s_{n}\right)$ ),

$\left(\mathrm{R}_{2}\right):\left(t_{n}, \theta_{n}\right)$ is compatible with $T_{i_{n}}$,

$\left(\mathrm{R}_{3}\right):$ If $s_{m}$ is the $R^{\left(\mu\left(s_{n}\right)\right)}$-predecessor of $s_{n}$, then

$\left\{\begin{array}{l}\text { - If } \mu\left(s_{m}\right)=\mu\left(s_{n}\right) \text { and } J\left(s_{m}\right) \subset I^{(+)}, \text {then } i_{m}=i_{n} \text { and } \theta_{m} \prec \theta_{n} . \\ \text { - If } J\left(s_{m}\right)=J\left(s_{n}\right) \subset I_{(\times)} \text {, then } i_{m}=i_{n} \text { and } \theta_{m} \prec \theta_{n} \text {. }\end{array}\right.$

By convention $s_{-1}=\emptyset$. Player II wins if he follows the rules forever.

Observe that if $J\left(s_{m}\right)=J\left(s_{n}\right)$, then $\mu\left(s_{m}\right)=\mu\left(s_{n}\right)$ but the converse is not true. Notice also that rule $\left(R_{3}\right)$ is coherent with rule $\left(R_{1}\right)$ : indeed by $\left(R_{1}\right)$ we already have $s_{m} \in S_{i_{m}}$ and if moreover $i_{m} \in I^{(+)}$, then $S_{i_{m}}$ is $R^{\left(\eta_{n}\right)}$-monotone and since $\xi_{i_{n}}=\xi_{i_{m}}$ and $s_{m} R^{\eta_{n}} s_{n}$, then $s_{n} \in S_{i_{m}}$, so $i_{m} \in J\left(s_{n}\right)$. Hence the choice $i_{n}=i_{m}$ respects rule $\left(\mathrm{R}_{1}\right)$. 
Fact 1. Suppose that $s_{m} R^{\left(\mu\left(s_{n}\right)\right)} s_{n}$. Then:

- either $\mu\left(s_{m}\right) \neq \mu\left(s_{n}\right)$ and hence $J\left(s_{m}\right) \cap J\left(s_{n}\right)=\emptyset$,

- or $\mu\left(s_{m}\right)=\mu\left(s_{n}\right), J\left(s_{m}\right) \subset I_{(\times)}$and $J\left(s_{n}\right) \subset I^{(+)}$and hence $J\left(s_{m}\right) \cap J\left(s_{n}\right)=\emptyset$,

— or $\mu\left(s_{m}\right)=\mu\left(s_{n}\right)$ and $J\left(s_{m}\right) \subset J\left(s_{n}\right) \subset I^{(+)}$,

— or $\mu\left(s_{m}\right)=\mu\left(s_{n}\right)$ and $J\left(s_{n}\right) \subset J\left(s_{m}\right) \subset I_{(\times)}$.

Proof. If $\mu\left(s_{m}\right) \neq \mu\left(s_{n}\right)$, then obviously $J\left(s_{m}\right) \cap J\left(s_{n}\right)=\emptyset$. So suppose that $\mu\left(s_{m}\right)=\mu\left(s_{n}\right)=\eta$.

- If $J\left(s_{m}\right) \subset I^{(+)}$, then for all $i \in J\left(s_{m}\right), S_{i}$ is $R^{(\eta)}$-monotone and since $s_{m} \in S_{i}$, then $s_{n} \in S_{i}$. Hence $\emptyset \neq J\left(s_{m}\right) \subset I\left(s_{n}\right) \cap I^{(+)}=J\left(s_{n}\right) \subset I^{(+)}$.

- If $J\left(s_{n}\right) \subset I(\times)$, then for all $i \in J\left(s_{n}\right), S_{i}$ is $R^{(\eta)}$-hereditary and since $s_{n} \in S_{i}$, then $s_{m} \in S_{i}$. Hence $J\left(s_{n}\right) \subset I\left(s_{m}\right)=J\left(s_{m}\right) \subset I(\times)$.

- Otherwise $J\left(s_{m}\right) \subset I(\times)$ and $J\left(s_{n}\right) \subset I^{(+)}$and again $J\left(s_{m}\right) \cap J\left(s_{n}\right)=\emptyset$.

It follows from Fact 1 that rule $\left(\mathrm{R}_{3}\right)$ can be reformulated by the following unique condition:

$\left(\mathrm{R}_{3}^{\prime}\right):$ If $s_{m}$ is the $R^{\left(\mu\left(s_{n}\right)\right)}$-predecessor of $s_{n}$ and $J\left(s_{m}\right) \subset J\left(s_{n}\right)$, then $i_{m}=i_{n}$ and $\theta_{m} \prec \theta_{n}$.

Initializing positions: Let $u$ be a I-position in $G$. We shall say that $u$ is an initializing position if

$$
J\left(s_{m}\right) \not \subset J\left(s_{n}\right),
$$

where $s_{n}$ denotes the last I-move in $u$ and $s_{m}$ denotes the $R^{\left(\mu\left(s_{n}\right)\right)}$-predecessor of $s_{n}$.

In particular if $u$ is an initializing position, then rule $\left(R_{3}\right)$ is irrelevant, and the next move in the game (which has to be made by Player II) can be of the form $(t, i, \emptyset)$ for any $i \in J\left(s_{n}\right)$.

By the same arguments as in Fact 1 one can easily prove the following result, which will be of very common use in the sequel.

Fact 2. Suppose that $s_{m} R^{(\eta)} s_{n}$.

- If $\mu\left(s_{m}\right) \leq \eta$ and $J\left(s_{m}\right) \subset I^{(+)}$, then $\mu\left(s_{n}\right) \leq \mu\left(s_{m}\right)$.

- If $\mu\left(s_{n}\right) \leq \eta$ and $J\left(s_{n}\right) \subset I(\times)$, then $\mu\left(s_{m}\right) \leq \mu\left(s_{n}\right)$.

Lemma 7.2. If Player II wins the game $G$, then $\left(B_{i}\right)_{i \in I}$ is weakly reducible to $\left(\omega^{\omega} \backslash C_{i}\right)_{i \in I}$.

Proof. A winning strategy $\sigma$ for Player II in the game $G$ defines a continuous mapping $F: x \mapsto\left(y,\left(i_{n}\right)_{n \in \omega},\left(\theta_{n}\right)_{n \in \omega}\right)$ from $\omega^{\omega}$ to $\omega^{\omega} \times I^{\omega} \times \operatorname{Seq}(\omega)^{\omega}$ such that for all $x$ and all $n, x_{\left.\right|_{n}}=\sigma\left(y_{\mid m}, i_{m}, \theta_{m}\right)_{m \leq n}$. So fix such a mapping $F$ and let $f: \omega^{\omega} \rightarrow \omega^{\omega}$ denote its first coordinate. We shall prove that $f$ is a weak reduction of $\left(B_{i}\right)_{i \in I}$ to $\left(\omega^{\omega} \backslash C_{i}\right)_{i \in I}$. For this fix $x \in \bigcup_{j \in \omega} B_{j}$; set $F(x)=\left(y,\left(i_{n}\right)_{n \in \omega},\left(\theta_{n}\right)_{n \in \omega}\right)$, so $y=f(x)$ and let:

$$
\eta=\min \left\{\xi_{i}: x \in B_{i}\right\} \text { and } I(\eta, x)=\left\{i \in I: \xi_{i}=\eta \text { and } x \in B_{i}\right\} .
$$

Finally let $\left(s_{n}^{(\eta)}\right)_{n \in \omega}$ be the increasing enumeration of $\hat{x}^{(\eta)}$ (the $R^{(\eta)}$-branch contained in $\hat{x}:=\left\{x_{\mid n}: n \in \omega\right\}$ ), and set $k_{n}:=\left|s_{n}^{(\eta)}\right|-1$ so that $s_{n}^{(\eta)}=s_{k_{n}}$ (with the notation used in the rules of the game above).

Consider then the set $H=\left\{j \in I_{(\times)}: \xi_{j}<\eta\right\}$ which, by the adequateness and regularity assumption, is finite. It follows from the minimality of $\eta$ that for all $j \in H, x \notin B_{j}$; hence the set $\hat{x}^{\left(\xi_{j}\right)} \cap S_{j}$ is finite and a fortiori the set $\hat{x}^{(\eta)} \cap S_{j}$ is 
finite too. Hence we can find $n_{0}$ such that for all $j \in H$ and all $n \geq n_{0}, s_{n}^{(\eta)} \notin S_{j}$. For the rest of the proof we distinguish the two cases:

- Case 1: $\quad I(\eta, x) \cap I^{(+)} \neq \emptyset$.

Pick any $j_{0} \in I(\eta, x) \cap I^{(+)}$: since $x \in B_{j_{0}}$, there exists some $m_{0}$ such that $s_{m_{0}}^{(\eta)} \in S_{j_{0}}$, and since $S_{j_{0}}$ is $R^{(\eta)}$-monotone, then $s_{n}^{(\eta)} \in S_{j_{0}}$ for all $n \geq m_{0}$. Finally let $n_{*}:=\max \left(n_{0}, m_{0}\right)$ and $j_{*}:=i_{k_{n_{*}}}$. It follows from rule $\left(\mathrm{R}_{1}\right)$ and from the choice of $n_{0}$ and $m_{0}$ that $\xi_{j_{*}}=\xi_{j_{0}}=\eta$; and since $j_{0} \in I\left(\eta, s_{n_{*}}^{(\eta)}\right) \cap I^{(+)}$, then $j_{*} \in I^{(+)}$, so $s_{n_{*}}^{(\eta)} \in S_{j_{*}}$ and $S_{j_{*}}$ is $R^{\eta}$-monotone; hence $x \in B_{j_{*}}$. Moreover since $j_{*} \in I^{(+)}$, then by the additive part of rule $\left(\mathrm{R}_{3}\right)$, for all $n>m \geq n_{*}$ we have $i_{k_{m}}=j_{*}$ and $\theta_{k_{m}} \prec \theta_{k_{n}}$ so $\theta=\bigcup_{m \geq n} \theta_{k_{m}} \in \omega^{\omega}$ and it follows from rule $\left(\mathrm{R}_{2}\right)$ that $(y, \theta) \in\left\lceil T_{j_{*}}\right\rceil$ and hence $y \in \omega^{\omega} \backslash C_{j_{*}}$.

- Case 2: $\quad I(\eta, x) \subset I_{(\times)}$.

Again pick any $j_{0} \in I(\eta, x) \subset I_{(\times)}$: since $x \in B_{j_{0}}$ and $S_{j_{0}}$ is $R^{(\eta)}$-hereditary, then $s_{n}^{(\eta)} \in S_{j_{0}}$ for all $n$; it follows that for all $n \geq n_{0}, \xi_{i_{k_{n}}}=\xi_{j_{0}}=\eta$. Hence by Fact 1 for all $n>m \geq n_{0}$ we have $I\left(\eta, s_{n}^{(\eta)}\right) \subset I\left(\eta, s_{m}^{(\eta)}\right) \subset I_{(\times)}$, and since these sets are finite there exists some $n_{*} \geq n_{0}$ such that $I\left(\eta, s_{n}\right)=I\left(\eta, s_{n_{*}}\right)$ for all $n \geq n_{*}$. Finally set $j_{*}:=i_{k_{n_{*}}}$. Then applying the multiplicative part of rule $\left(\mathrm{R}_{3}\right)$ one can prove as in Case 1 that $x \in B_{j_{*}}$ and $y \in \omega^{\omega} \backslash C_{j_{*}}$.

From now on we fix a winning strategy $\Sigma$ for Player $I$ in the game $G$. Our goal is to define explicitly from $\Sigma$ a $\boldsymbol{\Delta}_{1}^{1}$ covering $\left(A_{i}\right)_{i \in I}$ of type $\left(\boldsymbol{\Gamma}_{i}\right)_{i \in I}$ which is subordinate to $\left(C_{i}\right)_{i \in I}$.

Notation: For any $u \in \operatorname{Dom}\left(\mathrm{Ext}_{\mathrm{I}}\right)$ let:

$$
\tilde{s}(u)= \begin{cases}\emptyset & \text { if } u=\emptyset, \\ s_{n} & \text { if } u=\left(s_{0}, t_{0}, i_{0}, \theta_{0}, \ldots, s_{n}\right) .\end{cases}
$$

We also set:

$$
\tilde{\mu}(u):=\mu(\tilde{s}(u)) \quad \text { and } \quad \tilde{J}(u):=J(\tilde{s}(u)) .
$$

We then consider on $\operatorname{Dom}\left(\operatorname{Ext}_{\mathrm{I}}\right)$ the resolution family $\tilde{\mathcal{R}}=\left(\tilde{R}^{(\eta)}\right)_{\eta \leq \xi}$ defined by:

$$
u \tilde{R}^{(\eta)} v \Longleftrightarrow(u \preceq v) \text { and }\left(\tilde{s}(u) R^{(\eta)} \tilde{s}(v)\right) .
$$

Thus $\tilde{R}^{(0)}=\operatorname{Ext}_{\mathrm{I}}$ and identifying $G$ to a game on $\omega$ we can apply all the material of Section 15 to $\tilde{\mathcal{R}}$. In particular we shall consider the tree $\mathcal{T}$ and for $\tau \in \mathcal{T}$, the sets $\mathcal{S}^{\tau}$ and the mappings $\Phi^{\tau}$ introduced in Section 5.4. If $\tau \in \mathcal{T}$ with $\ell_{1}(\tau)=u$ we also set:

$$
\tilde{J}(\tau):=\tilde{J}(u)
$$

For all $y \in \omega^{\omega}$ let $\Sigma_{y}$ denote the set of all positions $v \in \Sigma$ which are compatible with $y$ in the sense that all of Player II's moves in $v$ are of the form $\left(y_{\left.\right|_{n}}, i, \theta\right)$. Since $\Sigma$ is a quasi-strategy (for Player I), then $\Sigma_{y}$ too is a quasi-strategy, that is, an $\tilde{R}^{(0)}$-strategical set, so we can define for all $\tau \in \mathcal{T}$ :

$$
\Sigma_{y}^{(\tau)}=\Phi^{\tau}\left(\Sigma_{y}\right) .
$$

We also set

$$
\Lambda_{y}^{(\tau)}=\left\{v \in \Phi^{\tau}\left(\Sigma_{y}\right): v \text { is a I-position }\right\} .
$$

We recall that by Theorem [5.5 if $\Sigma_{y} \in \mathcal{S}^{\tau}$ with $\ell(\tau)=(\eta, u)$, then $\Sigma_{y}^{(\tau)}$ is $\tilde{R}_{u}^{(\eta)}$ strategical. 
Definition 7.3. Let $\tau \in \mathcal{T}$ with $\ell_{1}(\tau)=u$. We shall say that $\tau$ is optimal for $y \in \omega^{\omega}$ if:

1) $u$ is an initializing position,

2) $\Sigma_{y} \in \mathcal{S}^{(\tau)}$

3) for all $v \in \Lambda_{y}^{(\tau)}, \tilde{J}(u) \subset \tilde{J}(v)$.

This notion will play a crucial role in the proof. Let us recall that an initializing position is by definition a I-position, hence nonempty; and a fortiori if $\tau \in \mathcal{T}$ is optimal for $y$, it is nonempty since $\ell_{1}(\tau) \neq \emptyset$. .

Lemma 7.4. If $\tau \in \mathcal{T}$ is optimal for $y$, then for all $i \in \tilde{J}(\tau), y \in C_{i}$.

Proof. Suppose that $\tau \in \mathcal{T}$ is optimal for $y$; set $(\eta, u)=\ell(\tau)$ and fix $i \in \tilde{J}(\tau)=$ $\tilde{J}(u)$. Observe first that if $|\tau|$ is odd, then $\tau^{*}$ is also optimal for $y$ since in this case $\mathcal{S}^{\left(\tau^{*}\right)}=\mathcal{S}^{(\tau)}$ and $\ell_{1}\left(\tau^{*}\right)=\ell_{1}(\tau)=u$. Hence we may suppose that $|\tau|$ is even.

We shall argue by contradiction: assuming that $y \notin C_{i}$ we shall construct an infinite run in the game $\left(\Sigma_{y}\right)^{(R)}$ (see Section 4.2) with $R=\tilde{R}_{u}^{(\eta)}$. Such a run induces canonically an infinite run in the game $G$ compatible with the strategy $\Sigma$, and this is absurd since $\Sigma$ is winning for Player I in the game $G$.

If $y \notin C_{i}$ we can fix $\theta \in \omega^{\omega}$ such that $(y, \theta) \in\left\lceil T_{i}\right\rceil$. Set $|u|=2 k+1$ and consider in the game $\left(\Sigma_{y}\right)^{(R)}$ the run in which, starting with $u_{0}=u$ for the first move, Player I chooses at each move some $u_{m} \in \Lambda_{y}^{(\tau)}$ to which Player II answers $a_{m}=\left(y_{\left.\right|_{k+1+m}}, i, \theta_{\mid m}\right)$. We have to check that all these moves are possible and legal in the game $\left(\Sigma_{y}\right)^{(R)}$.

- For $m=0$, observe that since $|\tau|$ is even, then by Theorem $5.5 \mathrm{~b}$ ), $u \in \Lambda_{y}^{(\tau)}$ and it follows from the definition of the relation $R=\tilde{R}_{u}^{(\eta)}$ that the $R$-predecessor of $u$ is $\emptyset$, so $u_{0}=u$ is a legal first move in $\left(\Sigma_{y}\right)^{(R)}$. Moreover since $i \in \tilde{J}(u)$ and $u$ is an initializing position, then $a_{0}=\left(y_{\left.\right|_{k+1+m}}, i, \emptyset\right)$ is a legal move for Player II in the game $\Sigma_{y}$, hence in $\left(\Sigma_{y}\right)^{(R)}$.

- Suppose that for $m>0$ the first $m$ moves of both players are legal in $\left(\Sigma_{y}\right)^{(R)}$. Then $u_{m-1} \in \Lambda_{y}^{(\tau)}$ and $u_{m-1}=v^{*}$ where $v$ is the II-position defined by $v=$ $u_{m-1}^{\widehat{L}}\left\langle a_{m-1}\right\rangle \in \Sigma_{y}$. Since $\Sigma_{y} \in \mathcal{S}^{\tau}$, then by Theorem [5.5 a), the set $\Sigma_{y}^{(\tau)}$ is $R$ strategical; hence we can find some $u_{m} \in \Lambda_{y}^{(\tau)}$ such that $v \prec u_{m-1}$ and $u_{m-1}$ is the $R$-predecessor of $u_{m}$. This proves the existence of $u_{m}$. Finally since $\tilde{J}\left(u_{0}\right) \subset \tilde{J}\left(u_{m}\right)$ it follows from rules $(\mathrm{R})$ and $\left(\mathrm{R}_{2}\right)$ of the game $G$ that $a_{m}=\left(y_{\left.\right|_{k+1+m}}, i, \theta_{\mid m}\right)$ is a legal move for Player II in the game $\Sigma_{y}$, hence in $\left(\Sigma_{y}\right)^{(R)}$.

So this run is infinite and this gives the contradiction.

If for a fixed $i$ we consider the set:

$$
\tilde{A}_{i}=\left\{y \in \omega^{\omega}: \exists \tau \in \mathcal{T} \text { optimal for } y \text { such that } i \in \tilde{J}(\tau)\right\},
$$

then Lemma 7.4 asserts that $\tilde{A}_{i} \subset C_{i}$, so the family $\left(\tilde{A}_{i}\right)_{i \in I}$ is subordinate to $\left(C_{i}\right)_{i \in I}$. In fact we shall prove that $\left(\tilde{A}_{i}\right)_{i \in I}$ is actually a covering of $\omega^{\omega}$. Also basing on Theorem [5.6 it is not difficult to see that $\tilde{A}_{i}$ is Borel: unfortunately the natural syntactical analysis of $\tilde{A}_{i}$ gives that this set is $\boldsymbol{\Sigma}_{1+\xi_{i}+1}^{0}$. Also our strategy will be to define a subset $A_{i} \subset \tilde{A}_{i}$ of lower complexity, namely $A_{i} \in \boldsymbol{\Gamma}_{i}$, and such that $\left(A_{i}\right)_{i \in I}$ is still a covering of $\omega^{\omega}$. More precisely we shall construct a mapping 
$y \mapsto \sigma_{y}$ from $\omega^{\omega}$ to $\mathcal{T}$ such that for all $y, \sigma_{y}$ is optimal for $y$ and then define:

$$
A_{i}=\left\{y: i \in \tilde{J}\left(\sigma_{y}\right)\right\} \subset \tilde{A}_{i} \subset C_{i} .
$$

Then $\left(A_{i}\right)_{i \in I}$ will be a subordinate covering to $\left(C_{i}\right)_{i \in I}$. The difficulty is that we will have to control simultaneously the pointwise condition " $\sigma_{y}$ is optimal for $y$ " and the global condition " $A_{i} \in \boldsymbol{\Gamma}_{i}$ ".

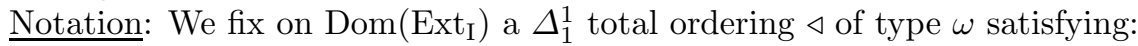

$$
u \prec v \Longrightarrow u \triangleleft v
$$

and equip the space $\omega_{1} \times \operatorname{Dom}\left(\operatorname{Ext}_{\mathrm{I}}\right)$ with the lexicographical product ordering $<_{\text {lex }}$ defined by:

$$
(\eta, v)<_{\operatorname{lex}}(\zeta, w) \Longleftrightarrow(\eta<\zeta) \text { or } \quad(\eta=\zeta \text { and } v \triangleleft w) \text {. }
$$

Also let

$$
\Omega:=\left\{\xi_{i}: i \in I_{(\times)}\right\} .
$$

Since $I_{(\times)}$was supposed to be infinite this set is infinite and since the Baire type $\left(\boldsymbol{\Gamma}_{i}\right)_{i \in I}$ is adequate, then $\Omega$ is of order type $\omega$, and for all $\eta<\xi$ we define

$$
\eta^{+}:=\min \left\{\eta^{\prime} \in \Omega: \eta<\eta^{\prime}\right\} .
$$

Definition 7.5. Set $\tau_{-1}=\emptyset$ and $\left(\eta_{-1}, v_{-1}\right)=(-1, \emptyset)$.

For any $y \in \omega^{\omega}$ let $\varphi(y)=\left(\eta_{m}, v_{m}, \tau_{m}\right)_{m}$ be the sequence with maximal domain defined inductively for $m \geq 0$ by:

$$
\left(\eta_{m}, v_{m}\right):=\text { the }<_{\text {lex }} \text {-minimum element }(\zeta, w) \in\left[\eta_{m-1}, \omega_{1}\left[\times \Lambda_{y}^{\left(\tau_{m-1}\right.} \smile\langle\zeta\rangle\right)\right.
$$

satisfying at least one of the following three conditions:

$$
\begin{aligned}
& \left\{\begin{aligned}
(i): & \quad " \eta_{m-1}=\tilde{\mu}\left(v_{m-1}\right)=\zeta \leq \tilde{\mu}(w), \tilde{J}\left(v_{m-1}\right) \not \subset \tilde{J}(w) ", \\
(i i): & \quad \eta_{m-1}<\tilde{\mu}\left(v_{m-1}\right), \quad \zeta=\tilde{\mu}(w)<\eta_{m-1}^{+}, \tilde{J}(w) \subset I^{(+)} ", \\
(i i i): & \quad \eta_{m-1}<\tilde{\mu}\left(v_{m-1}\right), \quad \zeta=\eta_{m-1}^{+} \leq \tilde{\mu}(w) ",
\end{aligned}\right. \\
& \tau_{m}=\left(\eta_{0}, v_{0}, \eta_{1}, \ldots \ldots, v_{m-1}, \eta_{m}, v_{m}\right) .
\end{aligned}
$$

Finally set

$$
\sigma_{y}=\bigcup_{m} \tau_{m}
$$

7.6. Remarks. This definition calls for some comments.

a) Let $(j)$ denote any of conditions $(i),(i i),(i i i)$ above. In the sequel we shall view such a condition as a formula $\psi^{(j)}\left(\eta_{m-1}, v_{m-1}, \zeta, w\right)$ with parameters $\eta_{m-1}, v_{m-1}$ and variables $\zeta, w$. Also when there is no ambiguity on the parameters $\eta_{m-1}, v_{m-1}$ we shall simply say that " $(\zeta, w)$ satisfies $(j)$ at level $m$ " to mean that $\psi^{(j)}\left(\eta_{m-1}, v_{m-1}, \zeta, w\right)$ holds.

b) Notice that in each of these conditions $\zeta$ is entirely determined by $w$ (and $\left.\eta_{m-1}\right)$ but the point is that we minimize with respect to the lexicographical ordering, hence first on $\zeta$ and then on $w$. This is namely the case in $(i i)$; however in $(i)$ and (iii), $\zeta$ is constant $\left(\zeta=\eta_{m-1}\right.$ or $\left.\eta_{m-1}^{+}\right)$, and in these two cases the minimization is just over $w$ with respect to the linear ordering $\triangleleft$.

c) On a more semantical level one can divide the positions $v_{m}$ occurring in $\sigma_{y}$ into two groups depending on whether: (1) $\eta_{m}=\mu\left(v_{m}\right)$ or $(2) \eta_{m}<\mu\left(v_{m}\right)$. As we shall see the role of the positions in the first group is to ensure that the sequence $\sigma_{y}$ is optimal for $y$, while positions of the second group are just coding 
some information about the values of the $\eta_{m}$ which will enable us to control some complexity computations later on.

From the previous definition we clearly have

$$
\eta_{m-1} \leq \eta_{m} \leq \mu\left(v_{m}\right) \text { and } v_{m-1} \preceq v_{m} ;
$$

in particular $\tau_{m} \in \mathcal{T}$, but as we shall see next we even have $v_{m-1} \tilde{R}^{\left(\eta_{m-1}\right)} v_{m}$.
Fact 3. a) $\Sigma_{y} \in \mathcal{S}^{\tau_{m}}$
b) $v_{m} \in \Lambda_{y}^{\left(\tau_{m}\right)}$;
c) for all $v \in \Lambda_{y}^{\left(\tau_{m}\right)}, v_{m} \tilde{R}^{\left(\eta_{m}\right)} v$.

Proof. a) Observe first that $\left|\tau_{m}\right|$ is even and $\ell_{1}\left(\tau_{m}\right)=v_{m}$; also from the definition of $\varphi$ we have $\left.v_{m} \in \Sigma_{y}^{\left(\tau_{m-1}\right.}-\left\langle\eta_{m}\right\rangle\right)=\Sigma_{y}^{\left(\tau_{m}^{*}\right)}$. It follows then by induction that $\Sigma_{y} \in \mathcal{S}^{\tau_{m}}$. b) Since $\left|\tau_{m}\right|$ is even this follows from Theorem $5.5 \mathrm{~b}$ ).

c) Observe that $\Sigma_{y}^{\left(\tau_{m}\right)} \subset\left(\Sigma_{y}\right)_{v_{m}}$; hence $v_{m}, v \in \Lambda_{y}^{\left(\tau_{m}\right)}$ with $v_{m} \tilde{R}^{(0)} v$, and the result follows from Theorem $5.5 \mathrm{c}$ ).

Lemma 7.7. For all $y$ the sequence $\sigma_{y}$ is finite.

Proof. Notice that if $\left(\eta_{m}, v_{m}\right)$ satisfies $(i)$, then $v_{m-1} \prec v_{m}$; and if it satisfies $(i i i)$, then $\eta_{m-1}<\eta_{m}$; finally if it satisfies $(i i)$ and $\eta_{m-1}=\eta_{m}$, then we have $\eta_{m-1}=$ $\eta_{m}=\tilde{\mu}\left(v_{m}\right)<\tilde{\mu}\left(v_{m-1}\right)$; and hence $v_{m-1} \prec v_{m}$. This proves that $\left(\eta_{m}, v_{m}\right) \neq$ $\left(\eta_{m-1}, v_{m-1}\right)$.

Assume now that $\sigma_{y}$ is infinite. Since all the $v_{m}$ are compatible with the fixed winning strategy $\Sigma$ for Player I in the game $G$, the sequence $\left(v_{m}\right)_{m}$ is eventually constant, say $v_{m}=v_{n}$ for $m \geq n$. Thus $\eta_{m-1}<\eta_{m}$ for $m>n$. So $\left(\eta_{m}, v_{m}\right)$ cannot satisfy $(i)$ for any $m>n$. Moreover if $\left(\eta_{m}, v_{m}\right)$ satisfies $(i i)$ for some $m>n$, then we would have

$$
\tilde{\mu}\left(v_{n}\right)=\tilde{\mu}\left(v_{m}\right)=\eta_{m}<\eta_{m+1} \leq \tilde{\mu}\left(v_{m+1}\right)=\tilde{\mu}\left(v_{n}\right),
$$

which is absurd. Hence $\left(\eta_{m}, v_{m}\right)$ satisfies $($ iii) for all $m>n$. It follows that $\eta_{m}=\eta_{m-1}^{+} \in \Omega$ and $\eta_{m-1}<\eta_{m} \leq \mu\left(v_{m}\right)=\mu\left(v_{n}\right)$ for each $m>n$. But since $\left\{\eta \in \Omega: \eta \leq \mu\left(v_{n}\right)\right\}$ is finite, this is impossible.

Lemma 7.8. For all $y$ the sequence $\sigma_{y}$ is optimal for $y$.

Proof. Fix $y$ and set $\varphi(y)=\left(\eta_{m}, v_{m}, \tau_{m}\right)_{m \leq n}$. In the sequel, $m$ denotes an arbitrary integer $\leq n$.

Fact 4. If $m<n$ and $\tilde{\mu}\left(v_{m}\right)=\eta_{m}$, then $\tilde{J}\left(v_{m}\right) \subset I(\times)$.

Proof. Suppose that for some $m<n$ we have $\tilde{\mu}\left(v_{m}\right)=\eta_{m}$ and $\tilde{J}\left(v_{m}\right) \subset I^{(+)}$. Then $v_{m+1}$ is necessarily defined by condition $(i)$; hence $\eta_{m+1}=\eta_{m} \leq \tilde{\mu}\left(v_{m+1}\right)$. But since $\tilde{J}\left(v_{m}\right) \subset I^{(+)}$and $v_{m} \tilde{R}^{\left(\eta_{m}\right)} v_{m+1}$, then (Fact 2) $\tilde{\mu}\left(v_{m+1}\right) \leq \tilde{\mu}\left(v_{m}\right)$. Hence $\tilde{\mu}\left(v_{m+1}\right)=\tilde{\mu}\left(v_{m}\right)$ and so $\left(\right.$ Fact 1) $\tilde{J}\left(v_{m}\right) \subset \tilde{J}\left(v_{m+1}\right)$, which contradicts $(i)$.

Fact 5. The set $\left\{\eta_{m}: m<n\right\}$ is an initial segment of $\Omega$.

Proof. Fix $m<n$ : by Fact 4 , $\left(\eta_{m}, v_{m}\right)$ cannot be defined by condition $(i i)$; hence: - If $\eta_{m-1}<\tilde{\mu}\left(v_{m-1}\right)$, then $\left(\eta_{m}, v_{m}\right)$ is defined by condition $(i i i)$; hence $\eta_{m}=$ $\eta_{m-1}^{+} \in \Omega$.

- If $\eta_{m-1}=\tilde{\mu}\left(v_{m-1}\right)$, then $\left(\eta_{m}, v_{m}\right)$ is defined by condition $(i)$ and $\eta_{m}=\eta_{m-1}$. But again by Fact 4 in this case we necessarily have $\tilde{J}\left(v_{m-1}\right) \subset I(\times)$ and hence $\eta_{m}=\eta_{m-1} \in \Omega$.

The conclusion follows then by a straightforward induction. 
Fact 6. For all $w \in \Lambda_{y}^{\left(\tau_{m}\right)}$ we have $\tilde{\mu}(w) \geq \eta_{m}$.

Proof. Assume that $w \in \Lambda_{y}^{\left(\eta_{m}\right)}$ is such that $\tilde{\mu}(w)<\eta_{m} \leq \tilde{\mu}\left(v_{m}\right)$. By Fact 3 we have $v_{m} \tilde{R}^{\left(\eta_{m}\right)} w$; hence $\left(\right.$ Fact 2) $\tilde{J}(w) \subset I^{(+)}$. Moreover since $\tau_{m}=\tau_{m-1}\left(\eta_{m}, v_{m}\right)$ and $\tilde{\mu}(w)<\eta_{m}$, then $w \in \Lambda_{y}^{\left(\tau_{m-1}\left\langle\eta_{m}\right\rangle\right)} \subset \Lambda_{y}^{\left(\tau_{m-1}\langle\tilde{\mu}(w)\rangle\right)}$. Finally by Fact 5 there exists a unique $k<m$ such that $\eta_{k} \leq \tilde{\mu}(w)<\eta_{k}^{+}=\eta_{k+1}$. This proves that $(\tilde{\mu}(w), w)$ satisfies condition $(i i)$ at level $k+1$.

Since $\eta_{k}<\eta_{k+1}$, then $\left(\eta_{k+1}, v_{k+1}\right)$ is not defined by condition $(i)$; by the previous observations, $\left(\eta_{k+1}, v_{k+1}\right)$ is necessarily defined by condition (ii). Hence $\left(\eta_{k+1}, v_{k+1}\right)$ satisfies condition $(i i)$ and so: $\tilde{J}\left(v_{k+1}\right) \subset I^{(+)}$and $\eta_{k} \leq \tilde{\mu}\left(v_{k+1}\right)<$ $\eta_{k}^{+}=\eta_{k+1}$; but since $v_{k+1} \tilde{R}^{\left(\eta_{k+1}\right)} v_{m}$, then (Fact 2) we have $\eta_{m} \leq \tilde{\mu}\left(v_{m}\right) \leq$ $\tilde{\mu}\left(v_{k+1}\right)<\eta_{k}^{+}=\eta_{k+1}$, which is impossible.

Fact 7. $v_{m}$ is an initializing position.

Proof. Let $v$ be the $\tilde{R}^{\left(\mu\left(v_{m}\right)\right)}$-predecessor of $v_{m}$; we have to show that $\tilde{J}(v) \not \subset \tilde{J}\left(v_{m}\right)$. Notice that since $\eta_{m} \leq \mu\left(v_{m}\right)$, then $v \tilde{R}^{\left(\eta_{m}\right)} v_{m}$. Let $k \leq m$ be the unique integer such that $v_{k-1} \preceq v \prec v_{k}$; since $v_{m} \in \Lambda_{y}^{\left(\tau_{m}\right)} \subset \Lambda_{y}^{\left(\tau_{k-1}\right)}$ and $v_{k-1} \preceq v \tilde{R}^{\left(\eta_{k-1}\right)} v_{m}$, then by Theorem $5.5 \mathrm{~d}), v \in \Lambda_{y}^{\left(\tau_{k-1}\right)}$. We now distinguish two cases:

- Case 1: $\eta_{k-1}=\tilde{\mu}\left(v_{k-1}\right)$.

In this case $\left(\eta_{k}, v_{k}\right)$ is necessarily defined by condition $(i)$. So $\eta_{k-1}=\eta_{k} \leq \tilde{\mu}\left(v_{k}\right)$ and since $v \prec v_{k}$, then by the $\triangleleft$-minimality of $v_{k}$ we necessarily have $\tilde{J}\left(v_{k-1}\right)=$ $\tilde{J}(v) \not \subset \tilde{J}\left(v_{k}\right)$ and a fortiori $\tilde{\mu}\left(v_{k-1}\right)=\tilde{\mu}(v)$; hence $\eta_{k-1}=\eta_{k}=\tilde{\mu}(v) \leq \eta_{m} \leq \tilde{\mu}\left(v_{m}\right)$. Moreover by Fact $4, \tilde{J}\left(v_{k-1}\right)=\tilde{J}(v) \subset I(\times)$.

Observe that if either $\tilde{J}\left(v_{m}\right) \subset I^{(+)}$or $\tilde{\mu}(v)<\tilde{\mu}\left(v_{m}\right)$, then obviously $\tilde{J}(v) \cap$ $\tilde{J}\left(v_{m}\right)=\emptyset$ and we are done. So we may assume that: (1) $\tilde{J}\left(v_{m}\right) \subset I_{(\times)}$and (2) $\tilde{\mu}(v)=\tilde{\mu}\left(v_{m}\right)$.

Consider now an arbitrary $j \in[k, m]$. If $j<m \leq n$, then it follows from Fact 4 that $\tilde{J}\left(v_{j}\right) \subset I_{(\times)}$, and by (1) this is also true for $j=m$; hence $\left(\eta_{j}, v_{j}\right)$ is not defined by condition (ii). On the other hand it follows from (2) that $\eta_{k-1}=\eta_{j-1}=$ $\eta_{j}=\eta_{m}$ so $\left(\eta_{j}, v_{j}\right)$ is not defined by condition $(i i i)$. Hence $\left(\eta_{j}, v_{j}\right)$ is defined by condition $(i)$ and we necessarily have $\eta_{j-1}=\tilde{\mu}\left(v_{j-1}\right)$; but again by $(2)$ we also have $\eta_{m}=\tilde{\mu}\left(v_{m}\right)$. This proves that for all $j \in[k, m]$ we have $\tilde{\mu}\left(v_{j}\right)=\tilde{\mu}\left(v_{j-1}\right)$ and $\tilde{J}\left(v_{j}\right) \not \subset \tilde{J}\left(v_{j-1}\right) \subset I(\times)$. Hence by Fact 1 we necessarily have: $\tilde{J}\left(v_{k-1}\right)=\tilde{J}(v) \supsetneq$ $\tilde{J}\left(v_{k-1}\right) \supsetneq \tilde{J}\left(v_{j}\right) \supsetneq \tilde{J}\left(v_{m}\right)$, which proves that $\tilde{J}(v) \not \subset \tilde{J}\left(v_{m}\right)$.

- Case 2: $\quad \eta_{k-1}<\tilde{\mu}\left(v_{k-1}\right)$.

In this case $\left(\eta_{k}, v_{k}\right)$ is defined either by condition (ii) or by condition (iii), but as we shall see the last alternative never occurs. Observe first that by Fact 6 , $\tilde{\mu}(v) \geq \eta_{k-1}$ and we now distinguish two subcases:

- Case 2.a: $\eta_{k-1} \leq \tilde{\mu}(v)<\eta_{k-1}^{+}$.

We claim that in this case $\tilde{J}(v) \subset \mathrm{I}^{(+)}$. For otherwise we would necessarily have $\tilde{\mu}(v)=\eta_{k-1}$ and since $v_{k-1} \tilde{R}^{\left(\eta_{k-1}\right)} v$, then by Fact 2 we would have $\tilde{\mu}\left(v_{k-1}\right) \leq$ $\tilde{\mu}(v)=\eta_{k-1}$, which contradicts the assumption of Case 2 .

So $\tilde{J}(v) \subset \mathrm{I}^{(+)}$and since $v \in \Lambda_{y}^{\left(\tau_{k-1}\right)}$, then $(\tilde{\mu}(v), v)$ satisfies condition $(i i)$ at level $k$ which proves that $\left(\eta_{k}, v_{k}\right)$ is actually defined by condition $(i i)$; hence $\tilde{\mu}\left(v_{k}\right)=\eta_{k}$ and $\left(\eta_{k}, v_{k}\right)<_{\operatorname{lex}}(\tilde{\mu}(v), v)$. Moreover by Fact 4 we necessarily have $k=m=n$. So 
$\tilde{\mu}\left(v_{m}\right)=\eta_{m}$ and $\left(\eta_{m}, v_{m}\right)<_{\operatorname{lex}}(\tilde{\mu}(v), v)$ and since $v \triangleleft v_{m}$, then $\eta_{m}=\tilde{\mu}\left(v_{m}\right)<\tilde{\mu}(v)$ and a fortiori $\tilde{J}(v) \cap \tilde{J}\left(v_{m}\right)=\emptyset$.

- Case 2.b: $\eta_{k-1}^{+} \leq \tilde{\mu}(v)$.

Again since $v \in \Lambda_{y}^{\left(\tau_{k-1}\right)}$, then in this case $\left(\eta_{k-1}^{+}, v\right)$ satisfies condition (iii). But we claim that $\left(\eta_{k}, v_{k}\right)$ is actually defined by condition $(i i)$. For otherwise it would be defined by condition $(i i i)$ and then $\eta_{k}=\eta_{k-1}^{+}$and $\left(\eta_{k-1}^{+}, v_{k}\right)<_{\text {lex }}\left(\eta_{k-1}^{+}, v\right)$, which is impossible since $v \triangleleft v_{k}$.

So $\left(\eta_{k}, v_{k}\right)$ is defined by condition $(i i)$. In particular $\tilde{\mu}\left(v_{k}\right)<\eta_{k-1}^{+}$and $\tilde{J}\left(v_{k}\right) \subset$ $\mathrm{I}^{(+)}$, and as in the previous case this implies that $k=m$. Hence $\tilde{\mu}\left(v_{m}\right)<\eta_{m-1}^{+} \leq$ $\tilde{\mu}(v)$ and again $\tilde{J}(v) \cap \tilde{J}\left(v_{m}\right)=\emptyset$.

Fact 8. $\tilde{\mu}\left(v_{n}\right)=\eta_{n}$ and $\sigma_{y} \neq \emptyset$.

Proof. Suppose by contradiction that $\eta_{n}<\tilde{\mu}\left(v_{n}\right)$; we shall show that one can extend the sequence $\varphi(y)$ by the induction scheme of Definition 7.5 by defining $\left(\eta_{n+1}, v_{n+1}\right)$, which will contradict the maximality of $\varphi(y)$. In fact it is sufficient to find some $(\zeta, w)$ satisfying one of conditions $(i),(i i)$ or $(i i i)$ at level $n+1$, but since $\tilde{\mu}\left(v_{n}\right)<\eta_{n}$, then condition $(i)$ will never be realized.

By Fact [3, $\Sigma_{y} \in \mathcal{S}^{\tau_{n}}$ and since $\left|\tau_{n}\right|$ is even, then $\Sigma_{y} \in \mathcal{S}^{\tau_{n}}\langle\xi\rangle$, so by Theorem 5.5 a) the set $\Sigma_{y}^{\left(\tau_{n}^{\frown}\langle\xi\rangle\right)}$ is $\tilde{R}_{v_{n}}^{(\xi)}$-strategical; hence it contains at least one I-position. So pick any $\left.w \in \mathrm{E}_{y}^{\left(\tau_{n}\right.}\langle\xi\rangle\right) \subset \Lambda_{y}^{\left(\tau_{n}\right)}$ : by Fact $\left[\right.$ we have $\tilde{\mu}(w) \geq \eta_{n}$ and we distinguish three cases:

- If $\tilde{\mu}(w)=\eta_{n}$, then since $v_{n} \tilde{R}^{\left(\eta_{n}\right)} w$ and $\tilde{\mu}(w)<\tilde{\mu}\left(v_{n}\right)$ we have (Fact 2) $\tilde{\mu}(w) \subset$ $I^{(+)}$; moreover $w \in \Lambda_{y}^{\left(\tau_{n}^{\Upsilon}\langle\xi\rangle\right)} \subset \Lambda_{y}^{\left(\tau_{n}^{\Upsilon}\langle\mu(w)\rangle\right)}$ and hence $(\mu(w), w)$ satisfies condition (ii).

- If $\eta_{n}<\tilde{\mu}(w)<\eta_{n}^{+}$, then $\mu(w) \notin \Omega$; hence $\tilde{\mu}(w) \subset I^{(+)}$and as in the previous case $(\mu(w), w)$ satisfies condition $(i i)$.

- If $\eta_{n}^{+} \leq \tilde{\mu}(w)$, then $w \in \Lambda_{y}^{\left(\tau_{n}^{-}\langle\xi\rangle\right)} \subset \Lambda_{y}^{\left(\tau_{n}\left\langle\eta_{n}^{+}\right\rangle\right)}$hence $\left(\eta_{n}^{+}, w\right)$ satisfies condition (iii).

In particular, since $\eta_{-1}=-1<0 \leq \tilde{\mu}\left(v_{-1}\right)$, the previous argument shows that $\left(\eta_{0}, v_{0}\right)$ is well defined, hence that $\sigma_{y} \neq \emptyset$.

\section{End of the proof of Lemma 7.8 :}

We have to show that $\sigma_{y}$ satisfies conditions 1), 2), 3) of Definition 7.3 . So set $u:=v_{n}$; by Fact 7, $u$ is an initializing position and by Fact 3, $\Sigma_{y} \in \mathcal{S}^{\tau}$. To finish suppose that for some $v \in \Lambda_{y}^{\left(\eta_{n}\right)}$ we have $\tilde{J}\left(v_{n}\right) \not \subset \tilde{J}(v)$; since by Fact 8 we have $\tilde{\mu}\left(v_{n}\right)=\eta_{n}$, then $\left(\eta_{n}, v\right)$ would satisfy condition $(i)$, which contradicts the maximality of $\varphi(y)$. This proves that $\sigma_{y}$ is optimal for $y$.

Notation: We recall that if $\tau \in \mathcal{T}$ with $\ell(\tau)=(\eta, u)$ we already defined $\tilde{J}(\tau):=$ $\tilde{J}(u)$; we also set

$$
\tilde{\mu}(\tau):=\tilde{\mu}(u) \quad \text { and } \quad \tilde{\nu}(\tau):=\eta
$$

Let $\mathcal{T}^{\circ}$ denote the set of all $\tau \in \mathcal{T}$ of even length. Notice that $\sigma_{y} \in \mathcal{T}^{\circ}$; moreover for any $\tau \preceq \sigma_{y}$ such that $\tau \in \mathcal{T}^{\circ}$ we have

$$
\tilde{\nu}(\tau) \leq \tilde{\mu}(\tau)
$$

Finally we define for all $i \in I$ :

$$
A_{i}=\left\{y \in \omega^{\omega}: i \in \tilde{J}\left(\sigma_{y}\right)\right\} .
$$


Lemma 7.9. $\left(A_{i}\right)_{i \in I}$ is a covering of Baire type $\left(\boldsymbol{\Gamma}_{i}\right)_{i \in I}$.

Proof. Since by definition $\tilde{J}\left(\sigma_{y}\right)$ is nonempty, $\left(A_{i}\right)_{i \in I}$ is clearly a covering of $\omega^{\omega}$ and all we have to prove is that for all $i, A_{i} \in \boldsymbol{\Gamma}_{i}$.

This proof will necessitate a number of descriptive complexity computations. But for practical reasons we will prefer to work with relations rather than sets. So given any pointclass $\boldsymbol{\Gamma}$ and any predicate $\Psi$ we shall say that "condition $\Psi(y)$ is $\boldsymbol{\Gamma}$ " or simply that " $\Psi$ is $\boldsymbol{\Gamma}$ " to mean that the set $\left\{y \in \omega^{\omega}: \Psi(y)\right\}$ is in $\boldsymbol{\Gamma}$. This abuse of terminology will not be confusing in the sequel since we shall never be concerned with the syntactical complexity of the formula $\Psi$.

Fact 9. For all $\tau \in \mathcal{T}^{\circ}$ there exists a $\boldsymbol{\Delta}_{1+\tilde{\nu}(\tau)}^{0}$ predicate $\Psi_{\tau}$ satisfying:

$$
\tau \preceq \sigma_{y} \Longrightarrow \Psi_{\tau}(y) \Longrightarrow\left\{\begin{array}{l}
\tau \preceq \sigma_{y} \\
\text { or } \\
\tilde{J}\left(\sigma_{y}\right) \subset I^{(+)} \text {and } \tilde{\mu}\left(\sigma_{y}\right)<\tilde{\nu}(\tau) .
\end{array}\right.
$$

Proof. We prove the result by induction on $|\tau|$.

We first set $\Psi_{\emptyset}:=" 0=0$ ", which satisfies obviously the statement. Consider now $\tau=\left(\eta_{0}, v_{0}, \ldots, \eta_{m}, v_{m}\right) \neq \emptyset$; set $\tau^{\prime}=\left(\eta_{0}, v_{0}, \ldots, \eta_{m-1}, v_{m-1}\right)=\tau_{|| \tau \mid-2}$ and suppose that $\Psi_{\tau^{\prime}}$ is already defined and satisfies the statement. Let $\varphi^{(j)}(\zeta, w)=$ $\psi^{(j)}\left(\eta_{m-1}, v_{m-1}, \zeta, w\right)$ be the formula defining condition $(j)$ in Definition 7.5 (see Remark (7.6) with parameters $\eta_{m-1}, v_{m-1}$. Observe that these formulas depend on the fixed $\tau$ and are not a priori involved in the definition of some $\sigma_{y}$. Now set $\varphi=\varphi^{(i)} \vee \varphi^{(i i)} \vee \varphi^{(i i i)}$ and define for all $y$ :

$$
\Psi_{\tau}(y):=" \Psi_{\tau^{\prime}}(y) \text { and } v_{m}=\min \left\{w: w \in \Lambda_{y}^{\left(\tau^{\prime} \succ\left\langle\eta_{m}\right\rangle\right)} \text { and } \varphi\left(\eta_{m}, w\right)\right\} ",
$$

where the minimum here is relative to the linear ordering $\triangleleft$.

By the induction hypothesis, condition " $\Psi_{\tau^{\prime}}(y)$ " is $\boldsymbol{\Delta}_{1+\eta_{m-1}}^{0}$, and by Theorem 5.6. condition " $w \in \Lambda_{y}^{\left(\tau^{\prime}\right.}\left\langle\left\langle\eta_{m}\right\rangle\right)$ " is $\boldsymbol{\Delta}_{1+\eta_{m}}^{0}$. Then noting that $v_{m}$ has finitely many predecessors (since the order $\triangleleft$ is of type $\omega$ ) one easily checks that " $\Psi_{\tau}(y)$ " is $\boldsymbol{\Delta}_{1+\eta_{m}}^{0}$.

To prove the implications of Fact 9 fix some $y$ and set $\sigma_{y}=\left(\zeta_{0}, w_{0}, \ldots, \zeta_{n}, w_{n}\right)$.

- If $\tau \preceq \sigma_{y}$, then it follows from the induction hypothesis that $\Psi_{\tau^{\prime}}(y)$ holds. Moreover since $\left(\zeta_{m-1}, w_{m-1}\right)=\left(\eta_{m-1}, v_{m-1}\right)$, then from the definition of $\sigma_{y}$ we have $\left(\eta_{m}, v_{m}\right)=\min \left\{(\zeta, w): w \in \Lambda_{y}^{\left(\tau^{\prime}-\langle\zeta\rangle\right)}\right.$ and $\left.\varphi(\zeta, w)\right\}$, so a fortiori $v_{m}=$ $\min \left\{w: w \in \Lambda_{y}^{\left(\tau^{\prime}-\left\langle\eta_{m}\right\rangle\right)}\right.$ and $\left.\varphi\left(\eta_{m}, w\right)\right\}$. This proves the first implication.

- We now prove the second implication; so suppose that $\Psi_{\tau}(y)$ holds. It follows then from the induction hypothesis that either " $\tau^{\prime} \preceq \sigma_{y}$ " or " $\tilde{J}\left(\sigma_{y}\right) \subset$ $I^{(+)}$and $\tilde{\mu}\left(\sigma_{y}\right)<\tilde{\nu}\left(\tau^{\prime}\right) "$, and in the latter case we are done since $\tilde{\nu}\left(\tau^{\prime}\right) \leq \tilde{\nu}(\tau)$. So we may assume that $\tau^{\prime} \preceq \sigma_{y}$, that is, $\left(\zeta_{k}, w_{k}\right)=\left(\eta_{k}, v_{k}\right)$ for all $k<m$. It also follows from $\Psi_{\tau}(y)$ that $\varphi\left(\eta_{m}, v_{m}\right)$ holds and we distinguish the three cases:

- Case 1: $\varphi^{(i)}\left(\eta_{m}, v_{m}\right)$ holds.

Since $\zeta_{m-1}=\eta_{m-1}=\tilde{\mu}\left(v_{m-1}\right)=\tilde{\mu}\left(w_{m-1}\right)$, then in the construction of $\sigma_{y}$, $\left(\zeta_{m}, w_{m}\right)$ is necessarily defined by condition $(i)$, and $\varphi_{\tau}^{(i)}\left(\eta_{m}, v_{m}\right)$ states precisely that $\left(\zeta_{m}, w_{m}\right)=\left(\eta_{m}, v_{m}\right)$; hence $\tau \preceq \sigma_{y}$.

- Case 2: $\varphi^{(i i)}\left(\eta_{m}, v_{m}\right)$ holds.

Since $\zeta_{m-1}=\eta_{m-1}<\tilde{\mu}\left(v_{m-1}\right)=\tilde{\mu}\left(w_{m-1}\right)$, then in the construction of $\sigma_{y}$, $\left(\zeta_{m}, w_{m}\right)$ is defined by either condition $(i i)$ or $(i i i)$, but since $\left(\eta_{m}, v_{m}\right)$ realizes $(i i)$ 
and is $<_{\text {lex }}$ smaller than any $\left(\zeta_{m}^{+}, w\right)$ satisfying $(i i i)$, then $\left(\zeta_{m}, w_{m}\right)$ is necessarily defined by condition $(i i)$; moreover:

$$
\zeta_{m-1}=\eta_{m-1} \leq \zeta_{m} \leq \eta_{m}<\eta_{m-1}^{+}=\zeta_{m-1}^{+} .
$$

- If $\zeta_{m}=\eta_{m}$, then $\varphi^{(i i)}\left(\eta_{m}, v_{m}\right)$ states precisely that $\left(\zeta_{m}, w_{m}\right)=\left(\eta_{m}, v_{m}\right)$; hence in this case $\tau \preceq \sigma_{y}$.

- If $\zeta_{m}<\eta_{m}$, then from the statement of condition $(i i)$ we have $\zeta_{m}=\tilde{\mu}\left(w_{m}\right)$ and $\tilde{J}\left(w_{m}\right) \subset I^{(+)}$; but then by Fact 4 we necessarily have $m=n$ and so $\tilde{J}\left(\sigma_{y}\right)=$ $\tilde{J}\left(w_{m}\right) \subset I^{(+)}$and $\tilde{\mu}\left(\sigma_{y}\right)=\tilde{\mu}\left(w_{m}\right)=\zeta_{m}<\eta_{m}=\tilde{\nu}(\tau)$.

- Case 3: $\varphi^{(i i i)}\left(\eta_{m}, v_{m}\right)$ holds.

Observe again that $\left(\zeta_{m}, w_{m}\right)$ is defined by either condition $(i i)$ or $(i i i)$ with $\zeta_{m} \leq \eta_{m}$. Then by the same arguments as in Case 2 one proves that if $\zeta_{m}=\eta_{m}$, then $\tau \preceq \sigma_{y}$, and if not, then $\tilde{J}\left(\sigma_{y}\right) \subset I^{(+)}$with $\tilde{\mu}\left(\sigma_{y}\right)=\zeta_{m}<\eta_{m}=\tilde{\nu}(\tau)$.

This finishes the proof of the second implication of Fact 9 .

Fact 10. If $i \in I^{(+)}$, then $A_{i}$ is $\boldsymbol{\Sigma}_{1+\xi_{i}}^{0}$.

Proof. We prove the fact by induction on $\xi_{i}$. So fix $i \in I^{(+)}$and suppose that for all $j \in I^{(+)}$such that $\xi_{j}<\xi_{i}, A_{j}$ is $\Sigma_{1+\xi_{j}}^{0}$. Since the Baire type is regular, then $\xi_{i}$ is necessarily a successor ordinal, say $\xi_{i}=\eta+1$, and it follows then from the induction assumption that the set:

$$
A^{+}\left(\xi_{i}\right):=\bigcup\left\{A_{j}: j \in I^{(+)}, \xi_{j}<\xi_{i}\right\}
$$

is $\boldsymbol{\Sigma}_{1+\eta}^{0} \subset \boldsymbol{\Delta}_{1+\xi_{i}}^{0}$. We claim that for all $y$ :

$$
\mathrm{E}^{(+)}: \quad y \in A_{i} \Longleftrightarrow \exists \tau \in \mathcal{T}^{\circ}: \tau \preceq \sigma_{y}, \tilde{\nu}(\tau)=\tilde{\mu}(\tau), i \in \tilde{J}(\tau) \subset I^{(+)} .
$$

To prove the implication from left to right, take $\tau=\sigma_{y}$, which by Fact 8 satisfies $\tilde{\nu}(\tau)=\tilde{\mu}(\tau)$. Conversely if $\tau$ is as on the right hand side of $\mathrm{E}^{(+)}$, then it follows from Facts 4 and 8 that we necessarily have $\tau=\sigma_{y}$; hence $i \in \tilde{J}\left(\sigma_{y}\right)$ and so $y \in A_{i}$.

Consider now the set $\hat{A}_{i}$ defined by:

$$
y \in \hat{A}_{i} \Longleftrightarrow \exists \tau \in \mathcal{T}^{\circ}: \Psi_{\tau}(y), \tilde{\nu}(\tau)=\tilde{\mu}(\tau), i \in \tilde{J}(\tau) \subset I^{(+)} .
$$

By Fact 9, $\hat{A}_{i}$ is $\boldsymbol{\Sigma}_{1+\xi_{i}}^{0}$; it also follows from the first implication of Fact 9 that $\hat{A}_{i} \supset A_{i}$ and from the second implication that $\hat{A}_{i} \subset A_{i} \cup A^{+}\left(\xi_{i}\right)$. But since $A^{+}\left(\xi_{i}\right)$ is disjoint from $A_{i}$, then $A_{i}=\hat{A}_{i} \backslash A^{+}\left(\xi_{i}\right)$ and hence $A_{i}$ is $\boldsymbol{\Sigma}_{1+\xi_{i}}^{0}$.

Fact 11. If $i \in I_{(\times)}$, then $A_{i}$ is $\mathbf{\Pi}_{1+\xi_{i}}^{0}$.

Proof. We prove the fact by induction on $\xi_{i}$. So fix $i \in I_{(\times)}$and suppose that for all $j \in I(\times)$ such that $\xi_{j}<\xi_{i}, A_{j}$ is $\boldsymbol{\Pi}_{1+\xi_{j}}^{0}$. It follows then from Fact 10] and from the induction hypothesis that the set

$$
A\left(\xi_{i}\right):=\bigcup\left\{A_{j}: j \in I, \xi_{j}<\xi_{i}\right\}
$$

is $\boldsymbol{\Sigma}_{1+\xi_{i}}^{0}$. We claim that for all $y$ :

$$
\mathrm{E}_{(\times)}: \quad y \notin A_{i} \Longleftrightarrow\left\{\begin{array}{l}
\tilde{\mu}\left(\sigma_{y}\right)<\xi_{i} \\
\text { or } \\
\exists \tau \in \mathcal{T}^{\circ}: \tau \preceq \sigma_{y}, \tilde{\nu}(\tau)=\xi_{i}, i \notin \tilde{J}(\tau) .
\end{array}\right.
$$


If $\tilde{\mu}\left(\sigma_{y}\right)<\xi_{i}$, then obviously $y \notin A_{i}$. On the other hand if $i \in \tilde{J}\left(\sigma_{y}\right) \subset I_{(\times)}$, then by Fact 1 for any $\tau \preceq \sigma_{y}$ such that $\tilde{\nu}(\tau)=\xi_{i}$ we necessarily have $i \in \tilde{J}\left(\sigma_{y}\right) \subset \tilde{J}(\tau)$. This proves the implication from right to left.

Suppose now that $y \notin A_{i}$, where $\sigma_{y}=\left(\eta_{0}, v_{0}, \ldots, \eta_{n}, v_{n}\right)$ is such that $\tilde{\mu}\left(\sigma_{y}\right) \geq \xi_{i}$. Then by Fact $8, \eta_{n}=\tilde{\mu}\left(\sigma_{y}\right) \geq \xi_{i}$; hence by Fact 5 there exists some $k$ such that $\eta_{k}=\xi_{i}$. Consider then the maximal $m \leq n$ such that $\eta_{m}=\xi_{i}$ and observe that since $y \notin A_{i}$, then necessarily $m<n$, or $m=n$ and $i \notin \tilde{J}\left(v_{m}\right)$. We claim that $i \notin \tilde{J}\left(v_{m}\right)$ : for otherwise we would have $\tilde{\mu}\left(v_{m}\right)=\xi_{i}=\eta_{m}$ and then in the construction of $\sigma_{y}$, $\left(\eta_{m+1}, v_{m+1}\right)$ should necessarily satisfy condition $(i)$ which implies that $\eta_{m+1}=$ $\eta_{m}$, and this contradicts the choice of $m$. Hence $\tau=\left(\eta_{0}, v_{0}, \ldots, \eta_{m}, v_{m}\right)$ satisfies $\tilde{\nu}(\tau)=\xi_{i}$ and $i \notin \tilde{J}(\tau)$. This proves the converse implication.

Thus if we denote by $A_{i}^{\prime}$ the set defined by the second line of the right-hand side of $\mathrm{E}_{(\times)}$, then we have $A_{i}^{c}=A_{i}^{\prime} \cup A\left(\xi_{i}\right)$ and if we consider the set $\hat{A}_{i}^{\prime}$ defined by

$$
y \in \hat{A}_{i}^{\prime} \Longleftrightarrow \exists \tau \in \mathcal{T}^{\circ}: \Psi_{\tau}(y), \tilde{\nu}(\tau)=\xi_{i}, i \notin \tilde{J}(\tau),
$$

then applying Fact 9 as in Fact 10 we get that $\hat{A}_{i}^{\prime}$ is $\boldsymbol{\Sigma}_{1+\xi_{i}}^{0}$ and $A_{i}^{\prime} \subset \hat{A}_{i}^{\prime} \subset A_{i}^{\prime} \cup$ $A^{+}\left(\xi_{i}\right)$; but since $A^{+}\left(\xi_{i}\right) \subset A_{i}^{c}$, then $A_{i}^{\prime}=\hat{A}_{i}^{\prime}$ and so $A_{i}^{c}=A_{i}^{\prime} \cup A\left(\xi_{i}\right)$ and hence $A_{i}^{c}$ is $\boldsymbol{\Sigma}_{1+\xi_{i}}^{0}$. This finishes the proof of Fact 11 .

The proof of Lemma 7.9 is now complete.

End of the proof of Theorem 7.1 .

- If Player II wins the game $G$, then by Lemma $7.2\left(B_{i}\right)_{i \in I}$ is weakly reducible to $\left(\omega^{\omega} \backslash C_{i}\right)_{i \in I}$, and the second alternative of Theorem 7.1 holds.

- If Player I wins the game $G$, then since this game is $\Delta_{1}^{1}$, by a classsical result (see [2], Theorem 35.32), Player I has a $\Delta_{1}^{1}$ strategy; so fix such a strategy $\Sigma$. Consider then the corresponding mapping $\varphi: \omega^{\omega} \rightarrow \mathcal{T}^{\circ}$ (as in Definition (7.5) and the family $\left(A_{i}\right)_{i \in I}$ defined above. It follows from Lemmas 7.4 and 7.8 that $A_{i} \subset C_{i}$ for all $i \in I$. Hence by Lemma 7.9. $\left(A_{i}\right)_{i \in I}$ is of type $\left(\boldsymbol{\Gamma}_{i}\right)_{i \in I}$ and is a subordinate covering to $\left(C_{i}\right)_{i \in I}$.

Finally observe that if $\alpha \in \omega^{\omega}$ is any $\Delta_{1}^{1}$ code for $\Sigma$, then all the previous construction can be achieved in $L^{\omega_{1}^{\alpha}}$ (see Remark 3.13). In particular the relation $R(\tau, y)$ defined by " $\Psi_{\tau}(y)$ " is clearly in $L^{\omega_{1}^{\alpha}}$. It follows then that the family $\left(A_{i}\right)_{i \in I}$ is $\Delta_{1}^{1}(\alpha)=\Delta_{1}^{1}$, which proves that the first alternative of Theorem 7.1 holds.

7.10. Remarks. The proof of Theorem 7.1 is the resultant of numerous attempts using simpler, but unfortunately incomplete, arguments; we strongly believe that one cannot bring drastic simplifications to this proof. Also we shall try through the following comments to throw some light on its technical aspects.

a) First of all observe that the reduction to the case where $\bigcup_{i \in I} S_{i}=\operatorname{Seq}(\omega)$ is unessential and one can easily repeat the proof without this additional assumption. The price to pay for this fake generality is that most of the definitions should include then one additional case which induces useless complications in the arguments.

However the reduction to the "regular" case is fundamental. More precisely Lemma 7.2 is false if the Baire type $\left(\boldsymbol{\Gamma}_{i}\right)_{i \in I}$ does contain an additive Baire class of limit rank (though by the reduction argument, Theorem 7.1 is still true).

b) The main difficulty in this proof lies in the fact that the Baire classes $\boldsymbol{\Gamma}_{i}$ that we handle are of different rank and nature (additive or multiplicative). For example if one restricts the study to Baire classes of constant rank or only to 
additive Baire classes $\boldsymbol{\Sigma}_{1+\xi_{i}}^{0}$, then many of the arguments become trivial (in the last case the sequence $\sigma_{y}$ of positions could be replaced by one position $v_{y}$ ). Most of the complications come from the treatment of the multiplicative Baire classes. A typical example is condition ( $i i i)$ in the definition of $\sigma_{y}$ whose only role is to avoid "skipping" any ordinal in $\Omega$ even if this ordinal is a priori irrelevant for the analysis of the fixed point $y$. This detail is crucial for the computation of the complexity of the sets $A_{i}$ when $i \in I^{(\times)}$while it does not interfere in the case $i \in I^{(+)}$(observe by the way that despite some formal similarities, the proofs of Fact 10 and Fact 11 are based on totally different arguments). Also quite surprisingly these complexity computations could be simplified in the case where the Baire type does not contain multiplicative Baire classes of limit rank; but this case cannot be eliminated by a reduction argument as for additive classes.

c) Theorem 7.1 possesses two aspects which are both nontrivial. On one hand it is quite clear that Theorem 7.1 is firstly an effectivity result, and in this respect even when restricted to Borel coverings this result is new and this additional assumption does not simplify any part of the previous proof. On the other hand for arbitrary $\boldsymbol{\Pi}_{1}^{1}$ coverings even the "boldface" version of Theorem 7.1 is new and again not simpler to prove.

Let us also point out that using Borel determinacy, Matrai obtains in [5] a similar boldface dichotomy for Borel partitions, in the particular case where the Baire type $\left(\boldsymbol{\Gamma}_{i}\right)_{i \in I}$ is a constant additive Baire class $\boldsymbol{\Sigma}_{\xi}^{0}$. In fact given any adequate Baire type $\left(\boldsymbol{\Gamma}_{i}\right)_{i \in I}$ one can replace the game $G$ used in the proof of Theorem 7.1 by a much simpler Borel version (in the spirit of Wadge games) and obtain very easily the same conclusion as Theorem 7.1 the sets $A_{i}$ can then be obtained (like in Wadge games) as the inverse images, by a winning strategy, of the sets $\omega^{\omega} \backslash C_{i}$ and the long construction of $\sigma_{y}$ as well as the descriptive computations via the predicates $\Psi_{\tau}$ are then no more needed. Unfortunately this soft approach based on Borel determinacy can neither give any effective information nor be applied to arbitrary $\boldsymbol{\Pi}_{1}^{1}$ coverings.

d) Finally observe that the statement of Theorem 7.1 is not a dichotomy in the sense that both alternatives might be realized simultanesouly. To obtain a real dichotomy one has to ensure that the testing family $\left(B_{i}\right)_{i \in I}$ is "really" of Baire type $\left(\boldsymbol{\Gamma}_{i}\right)_{i \in I}$ that is not "simpler", and as we shall see next this is always possible.

Proposition 7.11. For any Baire type $\left(\boldsymbol{\Gamma}_{i}\right)_{i \in I}$ of code $\alpha \in \omega^{\omega}$ there exists a $\Delta_{1}^{1}(\alpha)$ family $\left(B_{i}\right)_{i \in I}$ of type $\left(\boldsymbol{\Gamma}_{i}\right)_{i \in I}$ such that for any covering $\left(C_{i}\right)_{i \in I}$ at most one of the following two alternatives holds:

- either $\left(C_{i}\right)_{i \in I}$ admits a subordinate covering of type $\left(\boldsymbol{\Gamma}_{i}\right)_{i \in I}$,

- or $\left(B_{i}\right)_{i \in I}$ is weakly reducible to $\left(\omega^{\omega} \backslash C_{i}\right)_{i \in I}$.

Proof. By standard arguments we can find a $\Delta_{1}^{1}(\alpha)$ family $\left(V_{i}\right)_{i \in I}$ of type $\left(\boldsymbol{\Gamma}_{i}\right)_{i \in I}$ of subsets of $\omega^{\omega} \times \omega^{\omega}$ which is universal in the sense that for any family $\left(E_{i}\right)_{i \in I}$ of type $\left(\boldsymbol{\Gamma}_{i}\right)_{i \in I}$ of subsets of $\omega^{\omega}$ there exists some $a \in \omega^{\omega}$ such that for all $i \in I$ and all $x$ :

$$
x \in E_{i} \Longleftrightarrow(a, x) \in V_{i} .
$$

Consider then the "diagonal" family $\left(B_{i}\right)_{i \in I}$ defined by:

$$
B_{i}=\left\{x \in \omega^{\omega}:(x, x) \in V_{i}\right\}
$$

and suppose that $\left(C_{i}\right)_{i \in I}$ is a covering which satisfies both alternatives. So $\left(C_{i}\right)_{i \in I}$ admits a subordinate covering $\left(A_{i}\right)_{i \in I}$ of type $\left(\boldsymbol{\Gamma}_{i}\right)_{i \in I}$, and there exists a continuous 
mapping $f: \omega^{\omega} \rightarrow \omega^{\omega}$ which is a weak reduction $\left(B_{i}\right)_{i \in I}$ to $\left(\omega^{\omega} \backslash C_{i}\right)_{i \in I}$. Then the covering $\left(f^{-1}\left(A_{i}\right)\right)_{i \in I}$ is also of type $\left(\boldsymbol{\Gamma}_{i}\right)_{i \in I}$ and by the universality of $\left(V_{i}\right)_{i \in I}$ we can find $a \in \omega^{\omega}$ such that for all $i \in I, V_{i}(a)=f^{-1}\left(A_{i}\right)$. Hence for all $i \in I$ :

$$
a \in B_{i} \Longleftrightarrow(a, a) \in V_{i} \Longleftrightarrow a \in f^{-1}\left(A_{i}\right) \Longleftrightarrow f(a) \in A_{i} \Longrightarrow f(a) \in C_{i} .
$$

But since $\left(A_{i}\right)_{i \in I}$ is a covering we have $f(a) \in \bigcup_{i \in I} A_{i}$, so by the equivalences in (*) we also have $a \in \bigcup_{i \in I} B_{i}$. Hence there exists some $i_{*} \in I$ such that $a \in B_{i^{*}}$ and $f\left(a_{i^{*}}\right) \notin C_{i}$ and this contradicts $(\star)$.

Corollary 7.12. Let $\left(\boldsymbol{\Gamma}_{i}\right)_{i \in I}$ be an adequate Baire type of code $\alpha \in \omega^{\omega}$ and let $\left(C_{i}\right)_{i \in I}$ be a $\Pi_{1}^{1}(\alpha)$-covering. If $\left(C_{i}\right)_{i \in I}$ admits a subordinate covering of type $\left(\boldsymbol{\Gamma}_{i}\right)_{i \in I}$, then $\left(C_{i}\right)_{i \in I}$ admits a $\Delta_{1}^{1}(\alpha)$-subordinate covering of type $\left(\boldsymbol{\Gamma}_{i}\right)_{i \in I}$.

Proof. Apply Theorem 7.1 with $\left(B_{i}\right)_{i \in I}$ given by Proposition [7.11. Since $\left(C_{i}\right)_{i \in I}$ admits a subordinate covering of type $\left(\boldsymbol{\Gamma}_{i}\right)_{i \in I}$, then the second alternative of Theorem 7.1 cannot hold; hence the first alternative does hold. Consequently $\left(C_{i}\right)_{i \in I}$ admits a $\Delta_{1}^{1}(\alpha)$-subordinate covering of type $\left(\boldsymbol{\Gamma}_{i}\right)_{i \in I}$.

The following dual statement of Corollary 7.12 answers the initial question raised in the Introduction:

Corollary 7.13. Let $\left(\boldsymbol{\Gamma}_{i}\right)_{i \in I}$ be an adequate Baire type of code $\alpha \in \omega^{\omega}$ and let $\left(A_{i}\right)_{i \in I}$ be a $\Sigma_{1}^{1}(\alpha)$-family of subsets of $\omega^{\omega}$. If there exists a family $\left(B_{i}\right)_{i \in I}$ of type $\left(\boldsymbol{\Gamma}_{i}\right)_{i \in I}$ such that for all $i, A_{i} \subset B_{i}$ and $\bigcap_{i \in I} B_{i}=\emptyset$, then there exists such a family in $\Delta_{1}^{1}(\alpha)$.

Corollary 7.12 proves the implication $(i i i) \Rightarrow(i)$ of Theorem A quoted in the Introduction, and we now prove the second implication $(i i) \Rightarrow($ iii $)$.

\section{ON THE NECEssity OF ADEQUATE BAIRE TYPES}

Theorem 8.1. Let $\left(\boldsymbol{\Gamma}_{i}\right)_{i \in I}$ be a nonadequate Baire type of code $\alpha \in \omega^{\omega}$. Then there exists $\beta \in \omega^{\omega}$ and $a \Delta_{1}^{1}(\beta)$-covering $\left(C_{i}\right)_{i \in I}$ such that:

(1) $\left(C_{i}\right)_{i \in I}$ admits a subordinate covering of type $\left(\boldsymbol{\Gamma}_{i}\right)_{i \in I}$,

(2) $\left(C_{i}\right)_{i \in I}$ does not admit any $\Delta_{1}^{1}(\alpha, \beta)$-subordinate covering of type $\left(\boldsymbol{\Gamma}_{i}\right)_{i \in I}$.

Proof. Suppose that $\left(\boldsymbol{\Gamma}_{i}\right)_{i \in I}$ is of rank $\left(\xi_{i}\right)_{i \in I}$. By Proposition 6.5 we can find $i_{*} \in I$ and $\xi_{*}<\omega_{1}$ with code in $\Delta_{1}^{1}(\alpha)$ such that $\boldsymbol{\Gamma}_{i_{*}} \supset \boldsymbol{\Pi}_{\xi_{*}}^{0}$ and

$$
J_{*}=\left\{j \in I: \xi_{j} \leq \xi_{*} \text { and } \boldsymbol{\Gamma}_{j}=\boldsymbol{\Pi}_{\xi_{j}}^{0}\right\}
$$

is infinite. Then the Baire type $\left(\boldsymbol{\Gamma}_{i}\right)_{i \in\left\{i_{*}\right\} \cup J_{*}}$ is still nonadequate and has a code in $\Delta_{1}^{1}(\alpha)$, and if we can find a family $\left(C_{i}\right)_{i \in\left\{i_{*}\right\} \cup J_{*}}$ satisfying conditions (1) and (2) relative to $\left(\boldsymbol{\Gamma}_{i}\right)_{i \in\left\{i_{*}\right\} \cup J_{*}}$, then setting $C_{i}=\emptyset$ for $i \in I \backslash\left(\left\{i_{*}\right\} \cup J_{*}\right)$ we get a family $\left(C_{i}\right)_{i \in I}$ satisfying the same conditions relative to $\left(\boldsymbol{\Gamma}_{i}\right)_{i \in I}$.

Hence without loss of generality we can assume that $I=\omega, i_{*}=0, J_{*}=I \backslash\{0\}$ and $\xi_{*}=\sup \left\{\xi_{j}: j \in J_{*}\right\}$, that is:

$$
\begin{cases}\boldsymbol{\Gamma}_{i}=\boldsymbol{\Pi}_{\xi_{i}}^{0} & \text { for all } i>0, \\ \boldsymbol{\Gamma}_{0} \supset \boldsymbol{\Pi}_{\xi_{*}}^{0} & \text { with } \xi_{*}=\sup \left\{\xi_{i}: i>0\right\} .\end{cases}
$$

For practical reasons we shall not work directly in $\omega^{\omega}$ but in some homeomorphic copy $X$ which we will make precise later on. Also the definition of the sequence $\left(C_{i}\right)_{i \in \omega}$ will not be uniform: we shall first define $\left(C_{i}\right)_{i>0}$ explicitly, and then in 
second step choose a suitable $C_{0}$. In fact we shall introduce some parameter $T$ and define for all $T$ a $\Delta_{1}^{1}(T)$ set $C_{0}(T) \supset \omega^{\omega} \backslash \bigcup_{i>0} C_{i}$; then we shall prove that for some value $T_{0}$ of the parameter the sequence

$$
\left(C_{i}\right)_{i \in \omega}:=\left\langle C_{0}\left(T_{0}\right)\right\rangle \frown\left(C_{i}\right)_{i>0}
$$

satisfies conditions (1) and (2) with $\beta \approx\left(\alpha, T_{0}\right)$.

Let $\mathcal{T}$ denote the space of all trees on $\omega$ that we view as a $\Pi_{1}^{0}$ subset of the compact space $2^{\left({ }^{<\omega}\right)}$. The parameter $T$ will run a subset $\tilde{\mathcal{T}}$ of $\mathcal{T}$ that we describe now. For this consider on $\omega^{<\omega}$ the partial ordering $\leq$ defined by

$$
s \leq t \Longleftrightarrow|s|=|t|=n \text { and } \forall i<n, s(i) \leq t(i) .
$$

Then $\tilde{\mathcal{T}}$ will be the set of all trees $T$ on $\omega$ which are right hereditary with respect to this ordering, that is, satisfying

$$
s \in T \text { and } s \leq t \Longrightarrow t \in T .
$$

Let $\mathcal{T}_{\mathrm{WF}}$ denote the set of all well-founded trees on $\omega$ and set $\tilde{\mathcal{T}}_{\mathrm{WF}}:=\tilde{\mathcal{T}} \cap \mathcal{T}_{\mathrm{WF}}$. It is well known that the set $\mathcal{T}_{\mathrm{WF}}$ is $\Pi_{1}^{1}$-complete.

Lemma 8.2. $\tilde{\mathcal{T}}_{\mathrm{WF}}$ is $\Pi_{1}^{1}$-complete.

Proof. Consider the mapping $\Psi: \mathcal{T} \rightarrow \mathcal{T}$ defined by

$$
t \in \Psi(T) \Longleftrightarrow \exists s \in T: s \leq t .
$$

Since for all $t$ the set $\left\{s \in \omega^{<\omega}: s \leq t\right\}$ is finite, $\Psi$ is continuous; we shall prove that $\tilde{\mathcal{T}}_{\mathrm{WF}}=\Psi^{-1}\left(\mathcal{T}_{\mathrm{WF}}\right)$. First observe that $\Psi(\mathcal{T}) \subset \tilde{\mathcal{T}}$, so it is enough to check that $\mathcal{T}_{\mathrm{WF}}=\Psi^{-1}\left(\mathcal{T}_{\mathrm{WF}}\right)$.

If $\Psi(T)$ is well-founded, then a fortiori $T$ which is a subset of $\Psi(T)$ is wellfounded too. On the other hand if $\Psi(T)$ is ill-founded and possesses some infinite branch $\gamma$, then $T(\gamma):=\left\{t \in T: t \leq \gamma_{|| t \mid}\right\}$ is an infinite and finitely branching tree; hence $T(\gamma)$ admits an infinite branch and a fortiori $T$ is ill-founded too.

We now fix some more notation. Let $X:=\prod_{i>0} X_{i}$ with $X_{i}:=\omega^{\omega}$ for all $i>0$ and denote by $\pi_{i}: X \rightarrow X_{i}$ the canonical projections. Then clearly $X$ is homeomorphic to $\omega^{\omega}$ but to avoid any confusion we shall not identify these two spaces.

Fix some $\alpha \in \omega^{\omega}$ such that $\left(\xi_{i}\right)_{i \in \omega}$ admits a code in $\Delta_{1}^{1}(\alpha)$. We can then find a $\Delta_{1}^{1}(\alpha)$-family $\left(A_{i, k}\right)_{i>0, k \in \omega}$ of subsets of $\omega^{\omega}$ such that for all $i>0,\left(A_{i, k}\right)_{k \in \omega}$ is an increasing family of $\Delta_{\xi_{i}}^{0}$ sets with $A_{i, 0}=\emptyset$, and $A_{i}:=\bigcup_{k \in \omega} A_{i, k}$ is $\boldsymbol{\Sigma}_{\xi_{i}}^{0}$-complete. We then define for all $i>0$ and $k \in \omega$ :

$$
C_{i, k}:=\pi_{i}^{-1}\left(A_{i, k}\right) \quad \text { and } \quad C_{i}:=\pi_{i}^{-1}\left(A_{i}\right) .
$$

We now proceed to the definition of $C_{0}(T)$ for $T \in \tilde{\mathcal{T}}$. Consider first the mappings $\varphi_{i}: X \rightarrow \omega$ for $i \in \omega$ (including $n=0$ ), and $\varphi: X \rightarrow \omega^{\omega}$ defined by

$$
\varphi_{i}(x):= \begin{cases}0 & \text { if } x \notin C_{i+1}, \\ k+1 & \text { if } x \in C_{i+1, k+1} \backslash C_{i+1, k},\end{cases}
$$

and

$$
\varphi(x):=\left(\varphi_{i}(x)\right)_{i \in \omega}
$$


Finally set for all $T \in \tilde{\mathcal{T}}$ :

$$
\begin{aligned}
T^{*} & :=\left\{s \in \operatorname{Seq}(\omega): \forall m \leq|s|, s_{\left.\right|_{m}} \in T \text { or } \exists j<m, s(j)=0\right\}, \\
C_{0}(T) & :=\left\{x \in X: \forall n \in \omega, \varphi(x)_{\left.\right|_{n}} \in T^{*}\right\}, \\
\left(C_{i}(T)\right)_{i \in \omega} & :=\left\langle C_{0}(T)\right\rangle-\left(C_{i}\right)_{i>0} .
\end{aligned}
$$

We recall that $\xi_{*}=\sup _{i>0}\left(\xi_{i}\right)$ and we now set: $\xi^{*}=\sup _{i>0}\left(\xi_{i}+1\right)$.

Lemma 8.3. $\left(C_{i}(T)\right)_{i \in \omega}$ is a $\Delta_{1}^{1}(\alpha, T)$ covering of Baire type $\left\langle\boldsymbol{\Pi}_{\xi^{*}}^{0}\right\rangle-\left(\boldsymbol{\Sigma}_{\xi_{i}}^{0}\right)_{i>0}$.

Proof. If $x \in X \backslash \bigcup_{i>0} C_{i}$, then $\varphi_{i}(x)=0$ for all $i \in \omega$, so $x \in C_{0}(T)$. Hence $\left(C_{i}(T)\right)_{i \in \omega}$ is a covering which is clearly $\Delta_{1}^{1}(\alpha, T)$. Observe that $x \in C_{0}(T)$ if and only if:

$\forall i \in \omega, \quad\left(\exists j<i, x \notin C_{j}\right)$ or $\left(\exists s \in \omega^{i} \cap T, \forall j<i, x \in C_{j+1, s(j)+1} \backslash C_{j+1, s(j)}\right)$, which proves that $C_{0}(T)$ is $\Pi_{\xi^{*}}^{0}$. On the other hand, by construction, $C_{i}(T)=C_{i} \in$ $\Sigma_{\xi_{i}}^{0}$ for all $i>0$.

Lemma 8.4. If $T \in \tilde{\mathcal{T}} \backslash \tilde{\mathcal{T}}_{\mathrm{WF}}$, then the covering $\left(C_{i}(T)\right)_{i \in \omega}$ admits a subordinate covering of Baire type $\left(\boldsymbol{\Pi}_{\xi_{i}}^{0}\right)_{i \in \omega}$.

Proof. Fix some infinite branch $\gamma$ of $T$. Set $B_{i}:=C_{i, \gamma(i-1)}$ for all $i>0$, and $B_{0}:=\bigcap_{i>0} X \backslash C_{i, \gamma(i-1)}$. It is quite clear from these definitions that $\left(B_{i}\right)_{i \in \omega}$ is a covering of Baire type $\left(\boldsymbol{\Pi}_{\xi_{i}}^{0}\right)_{i \in \omega}$ and that $B_{i} \subset C_{i}$ for all $i>0$. Finally if $x \in B_{0}$, then for all $i \in \omega, \varphi_{i}(x) \geq \gamma(i)+1$; hence $\varphi(x)_{\left.\right|_{n}} \geq \gamma_{\left.\right|_{n}} \in T$. Since $T \in \tilde{\mathcal{T}}$, then $\varphi(x)_{{ }_{n}} \in T^{*}$; hence $x \in C_{0}(T)$. This proves that $\left(B_{i}\right)_{i \in \omega}$ is a subordinate covering to $\left(C_{i}(T)\right)_{i \in \omega}$.

Lemma 8.5. Let $\boldsymbol{\Gamma}$ be an arbitrary Baire class. If the covering $\left(C_{i}(T)\right)_{i \in \omega}$ admits a subordinate covering of Baire type $\langle\boldsymbol{\Gamma}\rangle \frown\left(\boldsymbol{\Pi}_{\xi_{i}}^{0}\right)_{i>0}$, then $T \in \tilde{\mathcal{T}} \backslash \tilde{\mathcal{T}}_{\mathrm{WF}}$.

Proof. Let us recall that $X=\prod_{i>0} X_{i}$ with each $X_{i}=\omega^{\omega}$. We can then fix a homeomorphism $h: X \rightarrow \omega^{\omega}$ with the property that if $x=\left(x_{i}\right)_{i>0}$ and $\gamma=h(x)$, then $\gamma_{\mid m}$ depends only on $\left(\left.x_{i}\right|_{m}\right)_{i \leq m}$.

Suppose now that $\left(B_{i}\right)_{i \in \omega}$ is a subordinate covering to $\left(C_{i}(T)\right)_{i \in \omega}$ of Baire type $\langle\boldsymbol{\Gamma}\rangle-\left(\boldsymbol{\Pi}_{\xi_{i}}^{0}\right)_{i>0}$ and fix $i>0$ in $\omega$. Then $h\left(B_{i}\right) \subset \omega^{\omega}$ is $\Pi_{\xi_{i}}^{0}$, and since $A_{i} \subset X_{i}=$ $\omega^{\omega}$ is $\Sigma_{\xi_{i}}^{0}$-complete, then in Wadge's game $G_{W}\left(A_{i}, h\left(B_{i}\right)\right)$ Player II cannot win; hence by the determinacy of this game Player I wins the game. Now any winning strategy for Player I induces a continuous mapping $\sigma_{i}: \omega^{\omega} \rightarrow X_{i}=\omega^{\omega}$ satisfying $h\left(B_{i}\right)=\sigma_{i}^{-1}\left(X_{i} \backslash A_{i}\right)$ and moreover for all $m \in \omega, \sigma_{i}(\gamma)_{\left.\right|_{m+1}}$ depends only on $\gamma_{\left.\right|_{m}}$.

Consider then the product mapping $\sigma: \omega^{\omega} \rightarrow X$ defined by

$$
\sigma(\gamma)=\left(\sigma_{i}(\gamma)\right)_{i>0} \in X=\prod_{i>0} X_{i}
$$

So $\pi_{i} \circ \sigma=\sigma_{i}: \omega^{\omega} \rightarrow X_{i}$ and $h \circ \sigma: \omega^{\omega} \rightarrow \omega^{\omega}$. It follows from the choice of $h$ and $\sigma_{i}$ that for all $m \in \omega,\left.h \circ \sigma(\gamma)\right|_{\left.\right|_{m+1}}$ depends only on $\gamma_{\left.\right|_{m}}$. Then one can define by induction sequences $u_{m} \in \omega^{m}$ such that

$$
\forall \gamma \quad\left(u_{m} \prec \gamma \Longrightarrow u_{m+1} \prec h(\sigma(\gamma))\right) .
$$

Since $u_{0}=\emptyset \prec u_{1}$, it is easily shown by induction that $u_{m} \prec u_{m+1}$ for all $m$. Then $\varepsilon=\bigcup_{i>0} u_{i}$ satisfies $h(\sigma(\varepsilon))=\varepsilon$ and if we set $x:=h^{-1}(\varepsilon)$, then

$$
\sigma(h(x))=\sigma(\varepsilon)=h^{-1}(\varepsilon)=x
$$


and for all $i>0$ :

$$
\pi_{i}(x)=\pi_{i}(\sigma(h(x)))=\sigma_{i}(h(x)) .
$$

Now observe that:

$x \notin C_{i} \Longleftrightarrow \pi_{i}(x)=\sigma_{i}(h(x)) \notin A_{i} \Longleftrightarrow h(x) \in h\left(B_{i}\right) \Longleftrightarrow x \in B_{i} \Longrightarrow x \in C_{i}$,

which proves that $x \in \bigcap_{i>0}\left(C_{i} \backslash B_{i}\right) \subset B_{0} \subset C_{0}(T)$.

It follows that for all $n \in \omega, \varphi(x)_{\left.\right|_{n}} \in T^{*}$; and since $x \in C_{i}$ then $\varphi_{i}(x)>0$, so necessarily $\varphi(x)_{\left.\right|_{n}} \in T$. Hence $\varphi(x)$ is an infinite branch of $T$ and so $T \in$ $\tilde{\mathcal{T}} \backslash \tilde{\mathcal{T}}_{\mathrm{WF}}$.

End of the proof of Theorem 8.1 Fix $\alpha \in \omega^{\omega}$ which codes $\left(\boldsymbol{\Gamma}_{i}\right)_{i \in \omega}$. Then clearly $\left(\xi_{i}\right)_{i \in \omega}$ admits a code in $\Delta_{1}^{1}(\alpha)$ and we can repeat the previous construction. Consider now the sets:

$$
\mathcal{S}:=\left\{T \in \tilde{\mathcal{T}}:\left(C_{i}(T)\right)_{i \in \omega} \text { admits a subordinate covering of type }\left(\boldsymbol{\Gamma}_{i}\right)_{i \in \omega}\right\},
$$

$\mathcal{S}_{0}:=\left\{T \in \tilde{\mathcal{T}}:\left(C_{i}(T)\right)_{i \in \omega}\right.$ admits a $\Delta_{1}^{1}(\alpha, T)$-subordinate covering of type $\left.\left(\boldsymbol{\Gamma}_{i}\right)_{i \in \omega}\right\}$.

Then clearly $\mathcal{S}_{0} \subset \mathcal{S}$ and we shall now prove that this inclusion is strict. Observe first that by a straightforward computation the set:

$$
\mathcal{U}:=\left\{(T, \gamma) \in \tilde{\mathcal{T}} \times \omega^{\omega}:\left(W_{i}^{\alpha}(\gamma)\right)_{i \in \omega} \text { is a subordinate covering for }\left(C_{i}(T)\right)_{i \in \omega}\right\}
$$

is $\Pi_{1}^{1}(\alpha)$; and since

$$
T \in \mathcal{S}_{0} \Longleftrightarrow \exists \gamma \in \Delta_{1}^{1}(\alpha, T),(T, \gamma) \in \mathcal{U},
$$

then $\mathcal{S}_{0}$ is also $\Pi_{1}^{1}(\alpha)$. On the other hand since $\boldsymbol{\Gamma}_{0} \supset \boldsymbol{\Pi}_{\xi_{*}}^{0}$, then by Lemmas 8.4 and 8.5 we have $\mathcal{S}=\tilde{\mathcal{T}} \backslash \tilde{\mathcal{T}}_{\mathrm{WF}}$; hence by Lemma 8.2 the set $\mathcal{S}$ is $\boldsymbol{\Sigma}_{1}^{1}$-complete.

It follows from these observations that $\mathcal{S} \backslash \mathcal{S}_{0} \neq \emptyset$ and if $T$ is any element of $\mathcal{S} \backslash \mathcal{S}_{0}$, then obviously $\left(C_{i}(T)\right)_{i \in \omega}$ satisfies the conclusion of Theorem 8.1 .

8.6. Remark. One cannot a priori impose on the element $T \in \mathcal{S} \backslash \mathcal{S}_{0}$ (chosen at the end of the previous proof) to be in $\Delta_{1}^{1}(\alpha)$. Also it seems quite unlikely that Theorem 8.1 could hold with $\beta=\alpha$. But one can obviously choose $T$, hence $\beta$, in any class which constitutes a basis for the class $\Sigma_{1}^{1}$; for example, applying the Gandy Basis Theorem one can choose $\beta$ such that $\omega_{1}^{\beta}=\omega_{1}^{\alpha}$.

\section{Proof of the Representation Theorem}

In this section we prove Theorem 3.10 stated in Section 3 , For this we need to construct resolution families $\left(R^{(\eta)}\right)_{\eta \leq \xi}$ which are strongly continuous at each limit level $\lambda \leq \xi$. Unfortunately the tree products technique used in [1] enables one to ensure the strong continuity condition only at the last level $\xi$ whenever this level is limit. So we have to describe a new method which keeps under control all intermediate limit levels.

In fact as in [1] we shall ensure a stronger condition than the strong continuity condition. This stronger condition that we introduce now is probably less intuitive but has proved to be efficient in several applications (see [1]).

We recall that if $R$ is a tree relation and $s$ an element of its domain, then $h_{R}(s)$ denotes the height of $s$ (i.e., the number of strict $R$-predecessors of $s$ ). 
Definition 9.1. Let $\mathcal{R}=\left(R^{(\eta)}\right)_{\eta \leq \xi}$ be a resolution family.

If $\lambda \leq \xi$ is limit ordinal we shall say that $\mathcal{R}$ is uniform at level $\lambda$ if for any integer $n$ there exists $\eta_{n}<\lambda$ such that for all $s$ and $t$ in $\operatorname{Dom}\left(R^{(0)}\right)$ with $\min \left(h_{R^{(\lambda)}}(s), h_{R^{(\lambda)}}(t)\right) \leq n$ we have

$$
s R^{\left(\eta_{n}\right)} t \Longrightarrow s R^{(\lambda)} t .
$$

We shall say that $\mathcal{R}$ is completely uniform if it is uniform at every limit level $\lambda \leq \xi$.

Clearly if $\mathcal{R}$ is uniform at some level $\lambda$, then it is strongly continuous at this level (if $n:=h_{R^{(\lambda)}}(s)$ and $\zeta:=\eta_{n}$, then $\left(s R^{(\zeta)} t \Rightarrow s R^{(\lambda)} t\right)$ ). Hence any completely uniform expansion family is strongly continuous. Observe also that "uniform" in the sense of 11 means exactly "uniform at the last level".

The following lemma will be the key for the construction of transfinite completely uniform expansion families.

Lemma 9.2. Let $\left(S^{(\eta)}\right)_{\eta \leq \xi}$ be a completely uniform expansion family, $\theta<\xi$ and $p \in \omega$. Define the family $\left(R^{(\eta)}\right)_{\eta \leq \xi}$ of relations by

$$
s R^{(\eta)} t \Longleftrightarrow\left(s S^{(\eta)} t\right) \text { or }\left(s S^{(\theta)} t \text { and } \eta \geq \theta \text { and } h_{S^{(\theta)}}(s) \leq p\right) \text {. }
$$

Then:

i) $\left(R^{(\eta)}\right)_{\eta \leq \xi}$ is a completely uniform expansion family, and $R^{(\eta)}=S^{(\eta)}$ if $\eta \leq \theta$.

ii) The canonical mapping $j_{\eta}:\left[S^{(\eta)}\right] \rightarrow\left[R^{(\eta)}\right]$ is a homeomorphism.

iii) For $\theta \leq \eta \leq \xi$ and $k \leq p$, we have $h_{R^{(\eta)}}(s)=k \Longleftrightarrow h_{S^{(\theta)}}(s)=k$.

iv) $\quad\left(h_{R^{(\xi)}}(s) \leq p\right.$ and $\left.s R^{(\theta)} t\right) \Longrightarrow s R^{(\xi)} t$.

Proof. i) It is readily seen that $R^{(\eta)}$ is a tree relation for all $\eta \leq \xi$, that $R^{(\eta+1)}$ is distinguished in $R^{(\eta)}$ for all $\eta<\xi$, that $R^{(\lambda)}=\bigcap_{\eta<\lambda} R^{(\eta)}$ for limit $\lambda \leq \xi$ and that $R^{(\eta)}=S^{(\eta)}$ for $\eta \leq \theta$.

We now prove that the canonical mapping $\pi_{\eta}^{\prime}:\left[R^{(\eta)}\right] \rightarrow\left[R^{(0)}\right]$ is onto for $\eta \leq \xi$ : for every $\sigma \in\left[S^{(\eta)}\right]$, both $\pi_{\eta}(\sigma)$ and $\pi_{\eta}^{\prime} \circ j_{\eta}(\sigma)$ are infinite $S^{(0)}$-branches containing $\sigma$; hence $\pi_{\eta}(\sigma)=\pi_{\eta^{\circ}}^{\prime} j_{\eta}(\sigma)$. Since $\pi_{\eta}^{\prime} \circ j_{\eta}=\pi_{\eta}$ is onto, then $\pi_{\eta}^{\prime}$ is onto.

Finally let $\lambda \leq \xi$ be a limit ordinal. We have to prove that the expansion family is uniform at level $\lambda$. It is clear if $\lambda \leq \theta$. If $\lambda>\theta$, then for any integer $n$ there is some $\eta_{n}<\lambda$, which can be assumed to be greater than $\theta$, such that for all $s$ and $t: \min \left(h_{S^{(\lambda)}}(s), h_{S^{(\lambda)}}(t)\right) \leq n$ and $s S^{\left(\eta_{n}\right)} t$ imply $s S^{(\lambda)} t$. But since $S^{(\eta)} \subset R^{(\eta)}$, we have $h_{S^{(\eta)}}(u) \leq h_{R^{(\eta)}}(u)$ for all $u$. Thus if $s$ and $t$ satisfy $s R^{\left(\eta_{n}\right)} t$ and $\min \left(h_{R^{(\lambda)}}(s), h_{R^{(\lambda)}}(t)\right) \leq n$, we have either $s S^{\left(\eta_{n}\right)} t$ and $\min \left(h_{S^{(\lambda)}}(s), h_{S^{(\lambda)}}(t)\right) \leq$ $n$, hence $s S^{(\lambda)} t$ and a fortiori $s R^{(\lambda)} t$, or $h_{S^{(\theta)}}(s) \leq p$ and $s S^{(\theta)} t$, hence $s R^{(\lambda)} t$.

ii) Since $S^{(\eta)} \subset R^{(\eta)}$, the mapping $j_{\eta}$ is continuous; it is bijective since $j_{\eta}=$ $\pi_{\eta}^{-1} \circ \pi_{\eta}^{\prime}$. To see that it is a homeomorphism it remains to show that for all $\eta \geq \theta$ and all $s$ in $\operatorname{Dom}\left(S^{(0)}\right)$ the set $E_{s}:=\left\{\sigma \in\left[R^{(\eta)}\right]: s \in J_{\eta}^{-1}(\sigma)\right\}$ is clopen. Denote by $W_{s}$ the clopen set $\left\{\sigma \in\left[R^{(\eta)}\right]: s \in \sigma\right\}$. Then since

$$
j_{\eta}^{-1}(\sigma)=\left\{t: \exists u \in \sigma \quad t R^{(\eta)} u\right\}=\sigma \cup\left\{t: h_{S^{(\theta)}}(t) \leq p \text { and } \exists u \in \sigma \quad t S^{(\theta)} u\right\} \text {, }
$$

one has $E_{s}=W_{s}$ or $E_{s} \cup \bigcup\left\{W_{u}: s S^{(\theta)} u\right\}$; hence $E_{s}$ is open. Similarly,

$$
\begin{aligned}
j_{\eta}^{-1}(\sigma) & =\left\{t: \forall u \in \sigma \quad u \preceq t \text { or } t R^{(\eta)} u\right\} \\
& =\sigma \cup\left\{t: h_{S^{(\theta)}}(t) \leq p \text { and } \forall u \in \sigma \quad h_{S^{(\theta)}}(u)>p \Longrightarrow t S^{(\theta)} u\right\} ;
\end{aligned}
$$


hence $E_{s}=W_{s}$ or $E_{s}=W_{s} \cup \bigcap\left\{W_{u}^{c}: h_{S^{(\theta)}}(u)>p\right.$ and $\left.\neg\left(s S^{(\theta)} u\right)\right\}$. Thus $E_{s}$ is closed.

iii) Let $\theta \leq \eta \leq \xi$. One has $R^{(\eta)} \subset S^{(\theta)}=R^{(\theta)}$; hence $h_{R^{(\eta)}}(s) \leq h_{S^{(\theta)}}(s)=$ $h_{R^{(\theta)}}(s)$. Thus $h_{R^{(\theta)}}(s)=k \leq p \Longrightarrow h_{R^{(\eta)}}(s) \leq k$. Conversely, if $h_{S^{(\theta)}}(s)=k \leq p$, there are pairwise distinct $\left(t_{i}\right)_{0 \leq i \leq k}$ such that $\emptyset=t_{0} S^{(\theta)} t_{1} S^{(\theta)} t_{2} \ldots S^{(\theta)} t_{k}=s$, and since $t_{i} R^{(\eta)} s$ for $i=1,2, \ldots, k$, we have $h_{R^{(\eta)}}(s) \geq k=h_{R^{(\theta)}}(s) \geq h_{R^{(\eta)}}(s)$.

iv) Assume that $k:=h_{R^{(\xi)}}(s) \leq p$. It follows from iii) that $h_{S^{(\theta)}}(s)=k \leq p$. If moreover $s R^{(\theta)} t$, we then have $s R^{(\xi)} t$.

Definition 9.3. a) We shall say that $\left(A_{i}, \xi_{i}\right)_{i \in I}$ is a graduate family of Borel sets if $A_{i} \in \mathbf{\Sigma}_{1+\xi_{i}}^{0}$ for all $i \in I$.

b) We shall say that the graduate family $\left(A_{i}, \xi_{i}\right)_{i \in I}$ of Borel sets is of level $\xi$ if $\sup _{i \in I} \xi_{i} \leq \xi$.

c) We shall say that the graduate family $\left(A_{i}, \xi_{i}\right)_{i \in I}$ of Borel sets is complete if:

(1) For all $i \in I$ such that $\xi_{i}$ is successor, there is a subset $J$ of $I$ such that $A_{i}=\bigcup_{j \in J} A_{j}^{c}$ and $\forall j \in J, \xi_{j}<\xi_{i}$.

(2) For all $i \in I$ such that $\xi_{i}$ is limit, there exists $J \subset I$ such that $\xi_{j}<\xi_{i}$ for all $j \in J$ and $A_{i}=\bigcup_{j \in J} A_{j}$.

We leave to the reader the proof of the following simple lemmas.

Lemma 9.4. Let $\left(A_{i}, \xi_{i}\right)_{i \in I}$ be a complete graduate family of Borel sets of level $\xi$. If $\zeta \leq \xi$ and $I_{\zeta}:=\left\{i \in I: \xi_{i} \leq \zeta\right\}$, the subfamily $\left(A_{i}, \xi\right)_{i \in I_{\zeta}}$ is complete of level $\zeta$.

Lemma 9.5. If $\left(B_{i}, \xi_{i}\right)_{i \in I}$ is a countable graduate family of Borel subsets of $\omega^{\omega}$ with $\xi:=\sup \xi_{i}$, then one can find a countable complete graduate family $\left(A_{j}, \eta_{j}\right)_{j \in J}$ of level $\xi$ such that $I \subset J$ and $\left(A_{i}, \eta_{i}\right)=\left(B_{i}, \xi_{i}\right)$ for $i \in I$.

Definition 9.6. Let $\left(A_{i}, \xi_{i}\right)_{i \in I}$ be a graduate family of Borel subsets of $\omega^{\omega}$. A strongly continuous expansion family $\left(R^{(\eta)}\right)_{\eta \leq \xi}$ is said to be adapted to $\left(A_{i}, \xi_{i}\right)_{i \in I}$ if, for all $i \in I, \pi_{\xi_{i}}^{-1}\left(A_{i}\right)$ is an open subset of $\left[R^{\left(\xi_{i}\right)}\right]$, where $\pi_{\xi_{i}}$ denotes the canonical mapping from $\left[R^{\left(\xi_{i}\right)}\right]$ into $[\mathrm{Ext}] \approx \omega^{\omega}$.

Theorem 9.7. Let $\left(B_{i}, \xi_{i}\right)_{i \in I}$ be a countable graduate family of Borel subsets of $\omega^{\omega}$, with $\xi=\sup _{i} \xi_{i}$. Then there exists a completely uniform expansion family $\left(R^{(\eta)}\right)_{\eta \leq \xi}$ adapted to $\left(B_{i}, \xi\right)_{i \in I}$.

Proof. Using Lemma 9.5 we can and do assume that $\left(B_{i}, \xi\right)_{i \in I}$ is complete of level $\xi$. We will prove by induction on $\xi$ the following statement.

$\left(H_{\xi}\right)$ : For any $\zeta \leq \xi$, any complete family $\left(A_{i}, \xi_{i}\right)_{i \in I}$ of level $\xi$ and any completely uniform expansion family $\left(R^{(\eta)}\right)_{\eta \leq \zeta}$ adapted to the subfamily $\left(A_{i}, \xi_{i}\right)_{i \in I_{\zeta}}$, there exists a completely uniform expansion family $\left(R^{(\eta)}\right)_{\eta \leq \xi}$ adapted to $\left(A_{i}, \xi_{i}\right)_{i \in I}$ and extending the given expansion family $\left(R^{(\eta)}\right)_{\eta \leq \zeta}$.

It is clear that Theorem 9.7 follows from this statement applied to $\zeta=0, R^{(0)}=$ Ext and $\left(A_{i}, \xi_{i}\right)_{i \in I}=\left(B_{i}, \xi_{i}\right)_{i \in I}$. Notice that if $\zeta=\xi$ there is nothing to prove. So we can always assume that $\zeta<\xi$.

a) The initial case: For $\xi=0$, we have necessarily $\zeta=\xi=0$ and there is nothing to prove.

b) The successor case: Assume that $H_{\xi}$ holds and let $\zeta<\xi+1,\left(A_{i}, \xi_{i}\right)_{i \in I}$ be a complete family of level $\xi+1$ and $\left(R^{(\eta)}\right)_{\eta \leq \zeta}$ a completely uniform expansion family adapted to the subfamily $\left(A_{i}, \xi_{i}\right)_{i \in I_{\zeta}}$. The statement $H_{\xi}$ applied to the 
family $\left(A_{i}, \xi_{i}\right)_{i \in I_{\xi}}$ enables us to assume that $\zeta=\xi$. We have then only to define the tree relation $R^{(\xi+1)}$ distinguished in $R^{(\xi)}$ in such a way that $\pi_{\xi+1}$ is onto and the set $\pi_{\xi+1}^{-1}\left(A_{i}\right)$ is open in $\left[R^{(\xi+1)}\right]$ for every $i \in I \backslash I_{\xi}$.

Observe that by completeness of the family, for each $i \in I \backslash I_{\xi}, \xi_{i}=\xi+1$ and there is a subset $J_{i}$ of $I_{\xi}$ such that $A_{i}=\bigcup_{j \in J_{i}} A_{j}^{c}$. Then $F_{j}:=\pi_{\xi}^{-1}\left(A_{j}^{c}\right)$ is closed in $\left[R^{(\xi)}\right]$ for all $j$ in $J_{i}$. Using Lemma I-6.3 of [1, we can find a tree relation $R$ distinguished in $R^{(\xi)}$ such that $\pi$ is onto and $\pi^{-1}\left(F_{j}\right)$ is $\Delta_{1}^{0}$ for each $j \in \bigcup_{i \in I \backslash I_{\xi}} J_{i}$ (where $\pi$ denotes the canonical mapping $[R] \rightarrow\left[R^{(\xi)}\right]$ ). Then putting $R^{(\xi+1)}:=R$, we have $\pi_{\xi+1}=\pi_{\xi \circ} \pi$; hence $\pi_{\xi+1}^{-1}\left(A_{i}\right)=\bigcup_{j \in J_{i}} \pi^{-1}\left(F_{j}\right) \in \boldsymbol{\Sigma}_{1}^{0}$.

c) The limit case: Assume that $\xi$ is limit and $H_{\theta}$ holds for all $\theta<\xi$. Choose a fundamental sequence $\left(\theta_{k}\right)$ for $\xi$. Let $\left(A_{i}, \xi_{i}\right)_{i \in I}$ be a complete family of level $\xi, \zeta<\xi$ and $\left(R^{(\eta)}\right)_{\eta \leq \zeta}$ be a completely uniform expansion family adapted to the subfamily $\left(A_{i}, \xi_{i}\right)_{i \in I_{\zeta}}$. Denote by $\ell$ the least integer $k$ such that $\zeta \leq \theta_{k}$.

We define inductively for $k \geq \ell$ completely uniform expansion families $\left(S_{k}^{(\eta)}\right)_{\eta \leq \theta_{k+1}}$ and $\left(R_{k}^{(\eta)}\right)_{\eta \leq \theta_{k+1}}$ in the following way:

- Applying $\left(H_{\theta_{\ell+1}}\right)$ we find $\left(S_{\ell}^{(\eta)}\right)_{\eta \leq \theta_{\ell+1}}$ extending $\left(R^{(\eta)}\right)_{\eta \leq \zeta}$ and adapted to the subfamily $\left(A_{i}, \xi_{i}\right)_{i \in I_{\theta_{\ell+1}}}$.

- Applying Lemma 9.2 we find $\left(R_{\ell}^{(\eta)}\right)_{\eta \leq \theta_{\ell+1}}$ which agrees with $\left(R_{\ell}^{(\eta)}\right)_{\eta \leq \theta_{\ell+1}}$ up to $\eta_{\ell}$ and satisfies properties i) to iv) of Lemma 9.2 for $(\theta, p)=\left(\theta_{\ell}, \ell\right)$.

- If $\left(S_{k}^{(\eta)}\right)_{\eta \leq \theta_{k+1}}$ and $\left(R_{k}^{(\eta)}\right)_{\eta \leq \theta_{k+1}}$ are defined for some $k \geq \ell$ we define $\left(S_{k+1}^{(\eta)}\right)_{\eta \leq \theta_{k+2}}$ extending $\left(R_{k}^{(\eta)}\right)_{\eta \leq \theta_{k+1}}$ by applying $\left(H_{\theta_{k+2}}\right)$, then $\left(R_{k+1}^{(\eta)}\right)_{\eta \leq \theta_{k+2}}$ by applying Lemma 9.2 to $\left(S_{k+1}^{(\eta)}\right)_{\eta \leq \theta_{k+2}}$ with $(\theta, p)=\left(\theta_{k+1}, k+1\right)$.

It is easy to check that $\left(R_{k+1}^{(\eta)}\right)_{\eta \leq \theta_{k+2}}$ extends $\left(R_{k}^{(\eta)}\right)_{\eta \leq \theta_{k+1}}$ for all $k$, hence that there is a family $\left(R^{(\eta)}\right)_{\eta<\xi}$ such that

$$
s R^{(\eta)} t \Longleftrightarrow s R_{k}^{(\eta)} t \text { for any } k \text { such that } \eta_{k+1} \geq \eta \text {. }
$$

Set $R^{(\xi)}=\bigcap_{k} R_{k}^{\left(\theta_{k}\right)}$. Clearly the family $\left(R^{(\eta)}\right)_{\eta \leq \xi}$ is an expansion family which is uniform at each limit level $\lambda<\xi$. It follows from the inductive construction that for each $i \in I$ such that $\xi_{i}<\xi$ the set $\pi_{\xi_{i}}^{-1}\left(A_{i}\right)$ is open in $\left[R^{\left(\xi_{i}\right)}\right]$.

It remains only to prove that $\left(R^{(\eta)}\right)_{\eta \leq \xi}$ is uniform at level $\xi$ and that $\pi_{\xi}^{-1}\left(A_{i}\right)$ is open in $\left[R^{(\xi)}\right]$ whenever $\xi_{i}=\xi$.

First observe that from the inductive construction we have: if $s$ and $t$ satisfy $h_{R^{\left(\theta_{k}\right)}}(s) \leq k$ and $s R^{\left(\theta_{k}\right)} t$, for some $k \geq \ell$, then for all $m \geq k$ :

$$
\begin{aligned}
s R^{\left(\theta_{m}\right)} t \text { and } h_{R^{\left(\theta_{m}\right)}}(s) & \leq m \Longrightarrow \\
& s R^{\left(\theta_{m+1}\right)} t \text { and } h_{R^{\left(\theta_{m+1}\right)}}(s) \leq h_{R^{\left(\theta_{m}\right)}}(s) \leq m \leq m+1
\end{aligned}
$$

and this proves by induction on $m$ that $s R^{(\xi)} t$.

We now prove that for $s \in \operatorname{Dom}\left(R^{(0)}\right)$ and $k \geq \ell, h_{R^{(\xi)}}(s) \leq k \Longleftrightarrow h_{R^{\left(\theta_{k}\right)}}(s) \leq$ $k$. Indeed assume that $h_{R^{\left(\theta_{k}\right)}}(s) \leq k$; since $R^{(\xi)} \subset R^{\left(\theta_{k}\right)}$, we have $h_{R^{(\xi)}}(s) \leq$ $h_{R^{\left(\theta_{k}\right)}}(s) \leq k$. Conversely, if $h_{R^{(\xi)}}(s) \leq k$ and $h_{R^{\left(\theta_{k}\right)}}(s)>k$, there are $t_{i} \neq s$ for $i \leq k$ such that $t_{i} R^{\left(\theta_{k}\right)} s$ and $h_{R^{\left(\theta_{k}\right)}}\left(t_{i}\right)=i$. It follows from what precedes that $t_{i} R^{(\xi)} t_{i+1}$, hence by induction on $i \leq k$ that $h_{R^{(\xi)}}\left(t_{i}\right) \geq i$. Finally we also have $t_{k} R^{(\xi)} s$, hence $h_{R^{(\xi)}}(s)>k$. 
Assume now that $\min \left(h_{R^{(\xi)}}(s), h_{R^{(\xi)}}(t)\right) \leq k$ and $s R^{\left(\theta_{k}\right)} t$. If $h_{R^{(\xi)}}(s) \leq k$ and $s R^{\left(\theta_{k}\right)} t$, we have $h_{R^{\left(\theta_{k}\right)}}(s) \leq k$ and $s R^{\left(\theta_{k}\right)} t$, hence $s R^{(\xi)} t$. On the contrary, if $h_{R^{(\xi)}}(t) \leq k$ and $s R^{\left(\theta_{k}\right)} t$, we have $h_{R^{\left(\theta_{k}\right)}}(t) \leq k$ and a fortiori $h_{R^{\left(\theta_{k}\right)}}(s) \leq k$; we conclude as above that $s R^{(\xi)} t$.

Finally if $i \in I$ with $\xi_{i}=\xi$, there is a subset $J$ of $I$ such that $A_{i}=\bigcup_{j \in J} A_{j}$ and $\xi_{j}<\xi$ for all $j$ in $J$. Since $R^{\left(\xi_{j}\right)} \subset R^{(\xi)}$, the canonical mapping $\pi_{\xi_{j}, \xi}$ is continuous from $\left[R^{(\xi)}\right]$ onto $\left[R^{\left(\xi_{j}\right)}\right]$, and the set $\pi_{\xi_{j}}^{-1}\left(A_{j}\right)$ is open in $\left[R^{\left(\xi_{j}\right)}\right]$. Thus $\pi_{\xi}^{-1}\left(A_{j}\right)=\pi_{\xi_{j}, \xi}^{-1}\left(\pi_{\xi_{j}}^{-1}\left(A_{j}\right)\right)$ is open in $\left[R^{(\xi)}\right]$, and so is $\pi_{\xi}^{-1}\left(A_{i}\right)=\bigcup_{j \in J} \pi_{\xi}^{-1}\left(A_{j}\right)$.

This completes the proof of Theorem 9.7.

Theorem 3.10 follows immediately from Theorem 9.7 since every completely uniform expansion family is strongly continuous.

\section{REFERENCES}

1. G. Debs and J. Saint Raymond, Borel liftings of Borel sets: Some decidable and undecidable statements, Memoirs of the Amer. Math. Soc. 187 (2007), no 876. MR2308388(2008b:03065)

2. A. S. Kechris, Classical Descriptive Set Theory, Graduate Texts in Mathematics, SpringerVerlag, New York, 1995. MR.1321597 (96e:03057)

3. A. Louveau, A separation theorem for $\Sigma_{1}^{\perp}$ sets, Trans. Amer. Math. Soc. 260-2 (1980) 363378. MR0574785 (81j:04001)

4. A. Louveau and J. Saint Raymond, Borel classes and closed games: Wadge-type and Hurewicz-type results, Trans. Amer. Math. Soc. 304-2 (1987) 431-467. MR0911079 (89g:03068)

5. T. Matrai, Hurewicz tests: Separating and reducing analytic sets on the conscious way, thesis, Central European University (2005).

6. Y. N. Moschovakis, Descriptive Set Theory, North Holland, Amsterdam, 1980. MR0561709 (82e:03002)

Analyse Fonctionnelle, Institut de Mathématique de Jussieu, Boîte 186, 4 Place Jussieu, F-75252 Paris Cedex 05, France

E-mail address: debs@math.jussieu.fr

Analyse Fonctionnelle, Institut de Mathématique de Jussieu, Boîte 186, 4 place Jussieu, F-75252 Paris Cedex 05, France

E-mail address: raymond@math.jussieu.fr 